\title{
Chapter
}

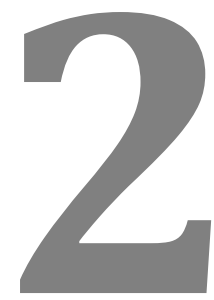

\section{APPLICATION OF HIGH PERFORMANCE LIQUID CHROMATOGRAPHY FOR THE MEASUREMENTS OF LIPOPHILICITY AND BIO-MIMETIC BINDING PROPERTIES IN DRUG DISCOVERY}

\author{
Klara Valko
}

\section{Contents}

2.2. THEORETICAL BACKGROUND OF HPLC.

2.2.1. Direct measurement of lipophilicity by chromatographic methods

2.2.2. Measurements of lipophilicity using isocratic chromatographic retention ......70

2.2.3. Measurements of lipophilicity using gradient chromatographic retention ......73

2.2.4. $\mathrm{pH}$ dependence of lipophilicity and reversed phase chromatographic retention

2. 3. APPLICATION OF BIO-MIMETIC HPLC STATIONARY PHASES.

2.3.1. Immobilised human serum albumin (HSA) stationary phase .79

2.3.2. Immobilised $\alpha$-1-acid-glycoprotein (AGP) stationary phase. 
2.4. GENERAL EXPERIMENTAL DESCRIPTION OF FAST GRADIENT HPLC ANALYSIS OF LIPOPHILICITY, PROTEIN AND PHOSPHOLIPID BINDING OF DRUG DISCOVERY COMPOUNDS .84

2. 4.1. Measurements of chromatographic hydrophobicity indices at three different $\mathrm{pHs}$ 84

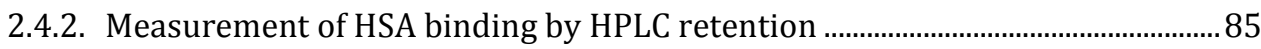

2.4.3. Measurement of AGP binding by HPLC retention ..................................................... 87

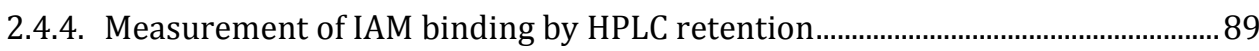

2.5. ANALYSIS OF THE HPLC BASED LIPOPHILICITY, PROTEIN BINDING AND

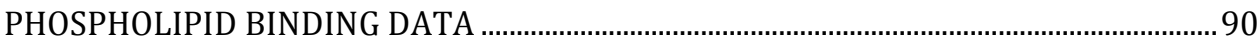

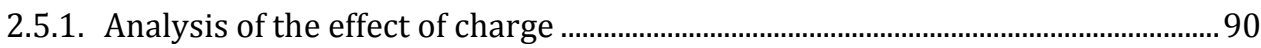

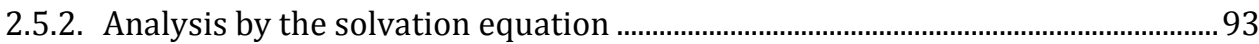

2.6. APPLICATIONS OF BIO-MIMETIC HPLC PROPERTIES TO MODEL DRUGS IN VIVO

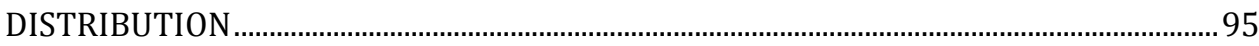

2.6.1. Modelling steady state volume of distribution ........................................................98

2.6.2. Modelling unbound volume of distribution ............................................................ 103

2.6.3. Dependence of unbound volume of distribution on HSA and IAM binding... 104

2.6.4. General structure-property relationships for lead optimisation ....................... 107

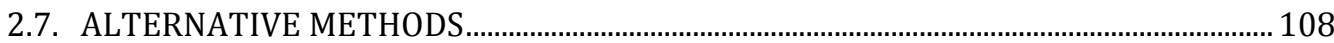

2.7.1. Methods based on the principle of frontal chromatography ............................... 108

2.7.2 Methods based on solid particles........................................................................... 111

2.7.3. Methods based on equilibrium dialysis and ultra-filtration ................................ 112

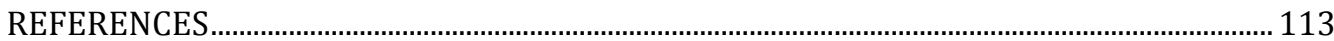




\subsection{INTRODUCTION}

One of the major concerns during the drug discovery process is that over $90 \%$ of potent candidate molecules fail during drug development. This is often due to the lack of efficacy in the clinical setting, unexpected side effects or unfavourable pharmacokinetics. It is widely accepted that unfavourable physico-chemical properties often contribute to these failures. Of the many recent reviews of the subject, one of the most well-known examples is the "Lipinski Rule of Five" [1], which indicates that solubility and permeability are the two critical properties for good oral absorption. Both solubility and permeability can be related to the size of the molecule, their lipophilicity and the number of $\mathrm{H}$-bond donor and acceptor groups. Since then, numerous publications have suggested that solubility, lipophilicity, permeability and the acid/base character are important factors that should be optimised in a candidate molecule in order to improve chances of drug molecule success [2-7]. There are several in silico prediction tools available [8-10] for chemists to estimate these important molecular physico-chemical properties before they synthesize compounds. However, there is always a need to have measured values from direct experimentation as well, as clinical chemistry delves into new chemical property areas where the in silico prediction tools are less accurate.

In general, we require methodologies for the measurement of solubility, lipophilicity, permeability and the acid/base character that are inexpensive, not labour intensive, and require small amounts of compound to obtain good, quality data.

Therefore, there is a need for physico-chemical property measurements of early discovery compounds that can provide fit-for-purpose accuracy with a fast turnaround time on a reasonably large number of compounds. We also need to be able to use these measured data to build structure-property relationships that can guide the medicinal chemistry efforts to find the best compromise of potency and physico-chemical properties. These properties can also be used for ranking and selecting compounds for more expensive and time consuming in vitro and in vivo measurements. The large amount of measured physico-chemical property data can also be used to improve in silico prediction tools by re-training the models. The optimised properties would provide the best chance for the compound to become a successful drug with the desired bioavailability and pharmacokinetic properties with the smallest possible dose and chance of toxic side effects. 
Early drug discovery compounds are usually new chemical entities for which we have very little information. Research compounds usually go through a generic High Performance Liquid Chromatography/Mass Spectrometry (HPLC/MS) analysis to determine the purity and identity of the compound before potency screening and further testing. HPLC technology is well adapted to cope with the analysis of large numbers of compounds. The fully automated procedure requires small amounts of compound and very little human intervention in providing the required information. It would be ideal if the same analysis technique could provide additional information other than purity and identity. It is well-known that in principle reversed phase retention is governed by the lipophilicity/hydrophobicity of compounds [11]; hence there is a possibility to obtain information about the compounds' lipophilicity at the same time as the quality control measurements are made. By altering the $\mathrm{pH}$ of the mobile phase and measuring the lipophilicity at various $\mathrm{pHs}$ via retention time we can provide a fast alternative to obtain information about the acid/base character of the compounds as well. The HPLC technique provides numerous advantages for the determination of the solubility of compounds. This involves the measurement of the concentration of the saturated solution which is in equilibrium with undissolved solid particles. The HPLC with UV detection, especially when it is coupled with mass spectrometry (MS), is one of the most popular generic methods that can be applied for fast, inexpensive and reasonably precise concentration determination. Thus, it is suitable to measure a compound's permeation through an artificial membrane. In conclusion, HPLC is very powerful not only as a separation and analytical method to determine purity and identity, but also as part of the methodology to determine various physico-chemical properties of drug discovery compounds such as lipophilicity, solubility, permeability and acid/base character. These properties can be used to build models for the estimation of the in vivo behaviour of the compounds and as selection criteria during the lead optimisation process. Applying these data in predictive models can help in the selection of molecules for further development at earlier stages of the lead optimisation process, thus reducing the chance of attrition in the clinical setting.

In this chapter the basic principles of chromatographic retention will be described along with how it can be used to derive various physico-chemical and binding properties of compounds. A short experimental description of the HPLC methodology will be provided for the determination of lipophilicity (including bio-mimetic lipophilicity), and the acid/base character of drug discovery compounds. Bio-mimetic chromatography as a powerful technique for measuring compound interactions with the major protein and phospholipids components in humans will also be explained. Finally, the application of the data will be demonstrated in various models used to predict human in vivo distribution. 


\subsection{THEORETICAL BACKGROUND OF HPLC}

HPLC is an established technique dating back to the 1960's. Several research journals, such as the Journal of Chromatography, Journal of Liquid Chromatography, Journal of Chromatographic Sciences, and Chromatographia publish regularly about the theory and application of HPLC. There are several books published for beginners in the field, with only a few cited here $[12,13]$, directing readers if they need more detailed knowledge about the principles of HPLC and running and troubleshooting HPLC experiments. Only the basic principles of HPLC that are necessary to understand the applicability of the method for physico-chemical measurements will be discussed here.

HPLC is a procedure where compounds are injected into a chromatographic system, which consists of a liquid mobile phase and a solid stationary phase. Compounds are separated due to differential migration in the chromatographic system depending on their interactions with the stationary and mobile phases. Components of a mixture injected into the system will move with the mobile phase but the speed of the component zones are different for each type of molecule in the mixture depending on their distribution between the mobile and stationary phases (see Figure 2.1).
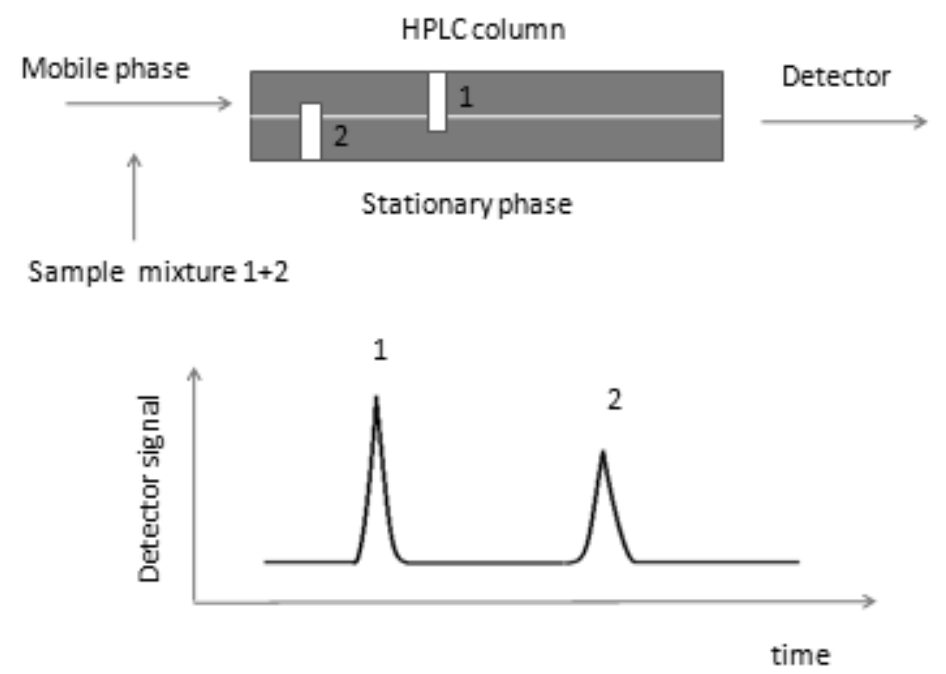

Figure 2.1. Schematic principles of chromatographic separation process

The mobile phase content is monitored at the end of the chromatographic column usually by UV detection. The detector signal is recorded as a function of time measured from the injection of the sample which takes place at the beginning of the chromatographic column. This column contains the stationary phase in the form of small particles. The recorded signal as a function of time is called a chromatogram. The chromatogram can show several peaks depending on the number of components in the mixture injected onto the column. The peak retention time 
(the time elapsed from the injection until the appearance of the maximum peak) is characteristic of the chemical nature of the components, while the peak area is proportional to the injected quantity. Thus, the chromatogram provides information both about the quantity and quality of the components in the mixtures. As the retention time depends upon the chromatographic conditions such as the type and composition of the stationary and mobile phases, the mobile phase flow rate, and the column dimensions, a known set of standard compounds should be analysed under identical conditions and then use the retention time itself for identification. Similarly, for quantification we need to inject a known amount of compound below a predetermined quantity and compare the peak area to the peak area of the unknown quantity of components in the samples. Nowadays, column dimensions of 30 to $50 \mathrm{~mm}$ in length and 2.1 to $3 \mathrm{~mm}$ diameter are used. The particle size of the stationary phase varies from 2 to $3 \mu \mathrm{m}$. The mobile phase flow rate is usually less than $1 \mathrm{ml}$ per minute.

In early drug discovery it is very common to use generic reversed phase gradient $[14,15]$ elution which involves silica stationary phase particles chemically bonded with $\mathrm{C}-18$ or C-8 ligand chemistry or polymer particles and aqueous acetonitrile or methanol as the mobile phase. The organic phase concentration changes during the analysis from a weaker - low organic phase concentration to a stronger - high organic phase concentration, thus ensuring that even strongly bound lipophilic compounds are eluted from the stationary phase in a single run and within a reasonably short time (usually 2 to 5 minutes).

Besides separation and concentration determination, the technique has great potential to determine partition properties of compounds based on their chromatographic retention times. The chromatographic retention time is directly related to the compound's distribution between the mobile and stationary phases. The retention factor $(k)$ determined from the retention time $\left(t_{R}\right)$ and dead time $\left(t_{0}\right)$ as $\left(t_{\mathrm{R}}-t_{0}\right) / t_{0}$ is equal to the ratio of the average number of analyte molecules in the stationary phase to the average number of molecules in the mobile phase (Equation 2.1) during the elution process.

$$
k=\frac{n_{\mathrm{s}}}{n_{\mathrm{m}}}=\frac{t_{\mathrm{r}}-t_{0}}{t_{0}}
$$

where $k$ is the retention factor, $n_{\mathrm{s}}$ is the average number of molecules in the stationary phase, $n_{\mathrm{m}}$ is the average number of molecules in the mobile phase.

The retention factor, $k$, can be related to the chromatographic partition coefficient of the compound, $K$, between the mobile and the stationary phase, according to Equation 2.2.

$$
\log k=\log K+\log \left(\frac{V_{\mathrm{s}}}{V_{\mathrm{m}}}\right)
$$

where $V_{\mathrm{s}} / V_{\mathrm{m}}$ is the volume ratio of the stationary phase and the mobile phase. 
Equation 2 shows that we need to know the value of the volume ratio of the stationary and mobile phases $\left(V_{\mathrm{s}} / V_{\mathrm{m}}\right)$ to be able to obtain the absolute value of the chromatographic partition coefficient $(\log K)$.

Equations 2.1 and 2.2 form the theoretical basis of deriving partition data from chromatographic retention. Thus, partition coefficients can be obtained from time measurements instead of concentration determination. The retention time is independent of the compound concentration/amount injected into the chromatographic system, and it is the main indicator of a true partition process. Impurities do not affect the measurements as they are usually separated from the main component. Low solubility also does not affect the measurements. The above advantages are very important in early drug discovery, where there are a large number of diverse compounds requiring analysis.

The chromatographic technique is very versatile as various stationary phases can be used to provide various types of partition information. For example, normal paraffin hydrocarbons, immobilised octanol, and bio-mimetic phases such as immobilised artificial membrane (IAM), human serum albumin (HSA), and alphaacid-glycoprotein (AGP) stationary phases can be used to reveal the compound's interaction/partition into a particular phase. The $\mathrm{pH}$ and polarity of the mobile phase can also be altered, which will affect compound interactions with the stationary phase too. The technique, therefore, is easily applicable for measurement of more than just octanol/water partitions. Since the advent of publications on chromatographic techniques [16-18] in the early 1970's, several hundred papers have been published about the applicability of reversed phase liquid chromatography for the determination of lipophilicity. From time to time, several review papers have summarised the major approaches [19-21].

Dorsey and Khaledi [22] have discussed the theoretical differences between chromatographic lipophilicity and partitioning between bulk solvents. They have also discussed the thermodynamic basis for possible failures in comparing the partitioning of small molecules between bulk solvents and chromatographic mobile and stationary phases, as well as the partitioning of solutes into lipid layers and biological membranes. Biological partition of compounds in aqueous bi-phase systems, such as blood and various tissues certainly happens in close to equal volume ratios involving a large area of contact surfaces between the partitioning phases. Therefore the chromatographic partition process is similar to the biological distribution processes. Significant differences between bulk solvent partition and partition involving large surfaces may be observed mainly for surface-active compounds, such as amphiphilic and charged molecules with large hydrophobic moieties.

In spite of the obvious advantages of HPLC, and the possibility of using biomimetic phases which may prove to be better models for biological partitions, the collation of HPLC based lipophilicity data is not widespread. The major problem in providing a more easily measurable alternative to the industry standard of octanol/water partition measurements lies in the variety of chromatographic 
stationary phases available and the lack of standardisation which make interlaboratory comparison of the data very difficult. To overcome these difficulties, the chromatographic system needs to be calibrated using known standards that also allow the chromatographic retention data to be converted into bio-mimetic partition coefficients. The reproducibility of current commercially available stationary phases and the automation and reliability of modern HPLC instrumentation now make it possible to fully exploit the advantages of the technique to collate a large amount of lipophilicity data in a reproducible way. However, it also requires consensus for standardisation.

\subsubsection{Direct measurement of lipophilicity by chromatographic methods}

HPLC provides a generally applicable approach for the determination of compound concentration. The equilibration of the compound in octanol and water can take place in an auto-sampler vial, 96 well plates or specially designed devices. By applying a generic fast gradient method, the equilibrium concentration of the compound in the two phases can be determined in minutes without the need for method development for each compound. The proportion of the peak areas obtained from the aqueous and the octanol phases can directly provide partition coefficients by taking into account the volumes of the two partitioning solvents. The biggest advantage of this approach is that it provides a true thermodynamic octanol/water partition coefficient. The HPLC technique is used for automatic determination of the concentration of the compound in the two partitioning solvents. In this respect, the result is independent of the chromatographic conditions used. An approximate amount of the compound is dissolved first in the octanol/saturated buffered water and the peak area of the solution is used as the "standard" concentration, $C_{\mathrm{s}}$, before partitioning. Various amounts of octanol are added to the aliquots of the sample solution, and the solutions are allowed to equilibrate before injecting again from the aqueous buffer layer, $C_{\mathrm{w}}$. The decrease of the peak area, $C_{\mathrm{s}}-C_{\mathrm{w}}$, indicates the amount of compound that partitioned to the octanol phase. Thus, the partition coefficient, $P$, can be obtained by Equation 2.3:

$$
P=\frac{c_{\mathrm{s}}-c_{\mathrm{w}}}{c_{\mathrm{w}}}=\frac{A_{\mathrm{s}}-A_{\mathrm{w}}}{A_{\mathrm{w}}}
$$

where $A_{s}$ is the peak area obtained from the aqueous buffer solution before equilibration with octanol, $A_{\mathrm{w}}$ is the peak area obtained from the aqueous buffer phase after equilibration with the octanol phase assuming we inject the same volume before and after the equilibration procedure [23]. Extra care has to be taken to make sure that the sample dissolves completely in the aqueous buffer before equilibration. Incomplete solubility during the equilibration provides false results for the partition coefficient. The volume of the two partitioning phases has to be significantly greater than the interface between the two solvents otherwise surface active compounds may accumulate at the interface and provide false partition coefficients. It is always advisable to repeat the measurements 
using various volume ratios of the two partitioning phases (octanol and aqueous buffer) and various amounts of sample and check the reproducibility of the results. Figure 2.2 shows an example of the HPLC chromatograms obtained from an initial aqueous stock solution and subsequent injections of the same compound after equilibration with various amounts of octanol.

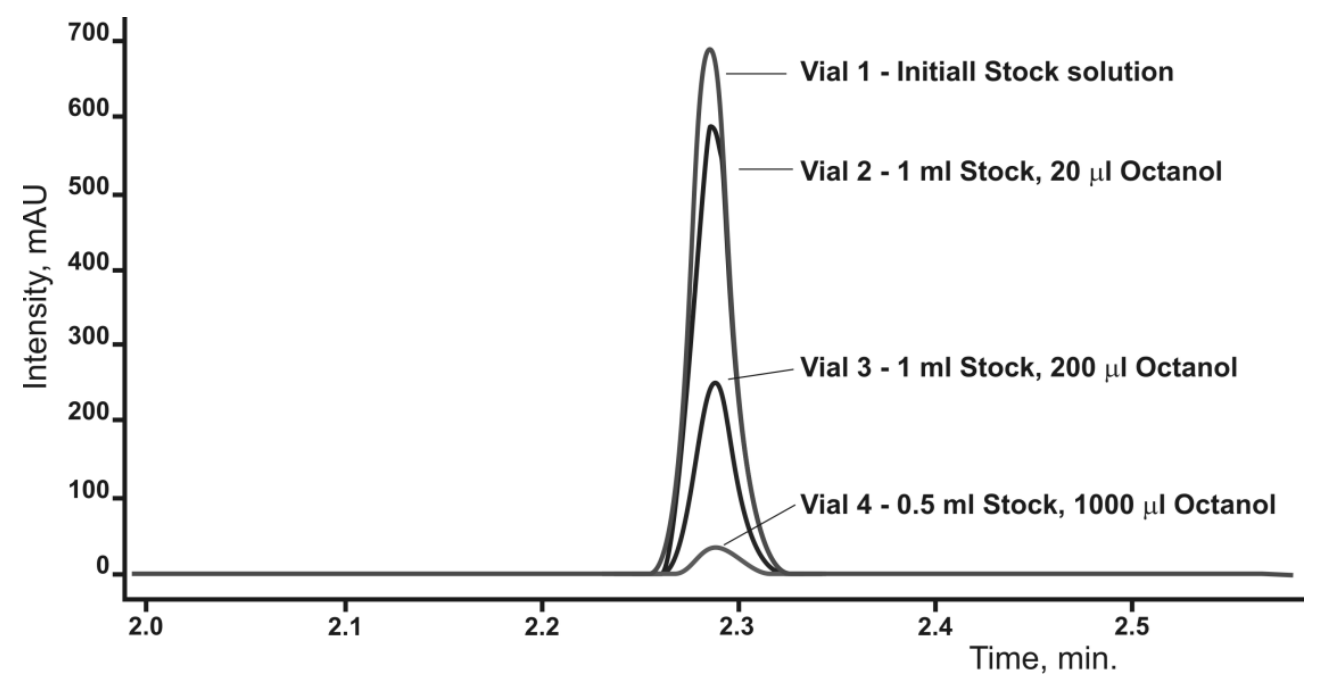

Figure 2.2. Chromatograms obtained from the initial aqueous buffer solution from a sample and after equilibration with various amounts of octanol

It is possible to use the chromatographic principle based on retention time measurements for the determination of octanol/water partition coefficients when we use octanol directly as the stationary phase and octanol saturated aqueous buffer as the mobile phase [24,25]. The difficulty of this approach is the immobilisation of octanol on the stationary phase surface, and keeping its volume constant during the elution process. Therefore, frequent calibration of the system is required. The chromatographic peaks are usually wide, which makes determination of retention time at the peak maximum variable, and the separation efficiency is low for possible impurities. The dynamic range of the precisely measurable octanol/water partition coefficients is small, as the octanol saturated mobile phase has low elution strength. In order to cover the wide range of octanol/water partition coefficients $(-1<\log P<5)$ the volume ratio of the octanol phase and water has to be changed.

Several attempts have been made to apply commercially available stationary phases and search for mobile phase additives that make the chromatographic partition system similar to the octanol/water partition system [26,27]. These approaches have the advantage of using commercially available efficient stationary phases. However, it is not certain that these systems behave exactly like octanol/water, especially in the case of ionised solutes. Although good agreement has been found between the chromatographic and equilibrium octanol/water partition data, deviations can be expected, as a large surface between 
the two partitioning phases and surface properties (surface activity, shape) can influence the interactions. For example, it was found [28] that negatively charged compounds did not show good correlation with the octanol/water distribution coefficients, even if the chromatographic partition was carefully tuned to model octanol/water partition.

\subsubsection{Measurements of lipophilicity using isocratic chromatographic retention}

HPLC is usually performed with high efficiency bonded reversed phase columns and today the commercially available columns are both robust and reproducible. Partitioning from aqueous/organic mobile phases into standard reversed phase (often C-18) HPLC stationary phases can be used as a direct measure of lipophilicity. In order to cover a wide range of lipophilicity, various concentrations of the organic solvent in the mobile phase must be used. Thus, it requires a preliminary estimate of the expected lipophilicity in order to choose the appropriate mobile phase composition. Several measurements at various mobile phase concentrations are needed for each compound resulting in longer processing time and data processing complications. To compare retention using different organic phase concentrations, they are extrapolated to the same condition, which is normally to zero organic solvent concentration. Equation 2.4 is used the most often for such an extrapolation.

$$
\log k=S \varphi+\log k_{\mathrm{w}}
$$

Equation 2.4 shows the linear relationship between the organic solvent concentration, $\varphi$, and the logarithmic retention factor. The relationship is not linear for the full range of organic solvent concentrations, but it is a good approximation within the working limits of $-0.5<\log k<1.5$. A linear plot of measured $\log k$ versus $\varphi$ provides the intercept $\left(\log k_{\mathrm{w}}\right)$, which is the extrapolated retention factor to zero organic phase concentration. In most cases, unless all the measurements are made on an identical HPLC column, it is also necessary to measure a standard set of compounds in order to calibrate the chromatographic system. Standardisation as suggested by the OECD guidelines [29], for example, is based on calibration using octanol/water $\log P$ values and the extrapolated $\log k_{\mathrm{w}}$ values. However, this is not a rigorously correct approach for two reasons. Firstly, the $\log k$ versus organic phase concentration plot is non linear, therefore different $\log k_{\mathrm{w}}$ values can be obtained for the same compound even when using the same column and instrument, when the $\log k_{\mathrm{w}}$ are derived using a different set of mobile phase compositions. Also different $\log k_{\mathrm{w}}$ values are obtained when acetonitrile or methanol is used as an organic modifier. Secondly, the reversed phase chromatographic system does not model well the octanol/water system for structurally diverse compounds.

It has been demonstrated [30] that the straight lines obtained by Equation 2.4 can cross each other (see Figure 2.3), hence, different lipophilicity rankings are obtained at different organic solvent concentrations. 


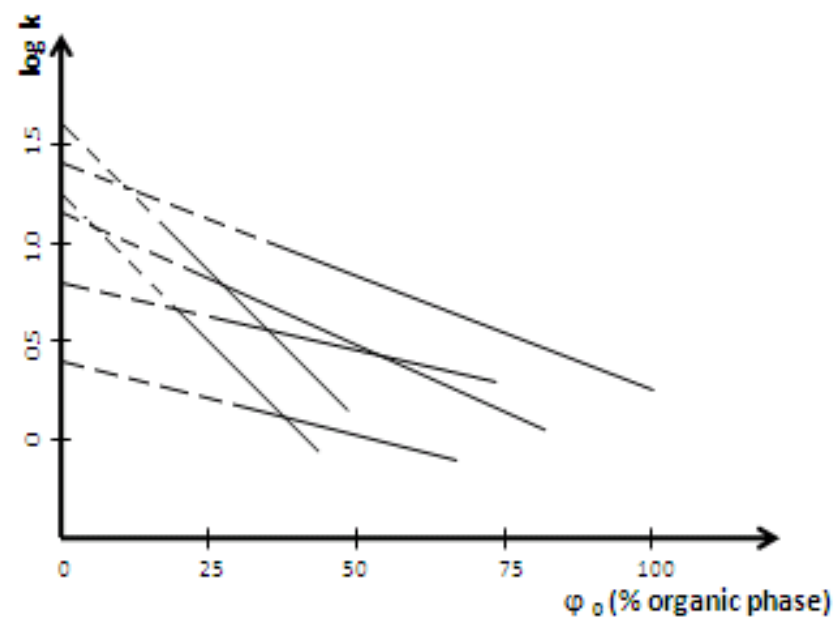

Figure 2.3. The hypothetical straight lines obtained for five compounds by plotting the logarithmic retention factor ( $\log k$ ) against the organic phase concentration in the mobile phase $(\varphi)$. The dotted lines show the extrapolation to zero organic phase concentration $\left(\log k_{\mathrm{w}}\right)$

The best correlation with the octanol/water partition coefficients is not necessarily achieved at the zero organic phase concentration, therefore the $S$ value in Equation 2.4 can be used as a second independent variable together with the log $k_{\mathrm{w}}$ values (Equation 2.5 ).

$$
\log P=a S+b \log k_{\mathrm{w}}+c
$$

where $a, b$ and $c$ are regression coefficients. The ratio of $a$ to $b$ gives an organic modifier concentration value, to which the $\log k$ values should be extrapolated to give the best statistical correlation to the $\log P$ values. In this way the chromatographic partition can be tuned to better model the octanol/water partition. With the improvements in stationary phases, where the masking of the free silanol groups is very successful, the $S$ and $\log k_{\mathrm{w}}$ values show quite good correlation, which means the same retention order can be obtained at various organic phase concentrations. Thus, the ability to tune the reversed phase chromatographic system to model the octanol/water partition system is greatly reduced.

The quotient of the intercept and slope in Equation $2.4\left(-\log k_{\mathrm{w}} / S=\varphi_{0}\right)$ also shows better correlation with the $\log P$ values than $\log k_{\mathrm{w}}$ [31] as is shown by Equation 2.6.

$$
\log P=d \varphi_{0}+e
$$

where $d$ and $e$ are regression coefficients.

The $\varphi_{0}$ is the $x$-coordinate of the intersection of the $\log k v s . \varphi$ lines as demonstrated in Figure 2.4. 


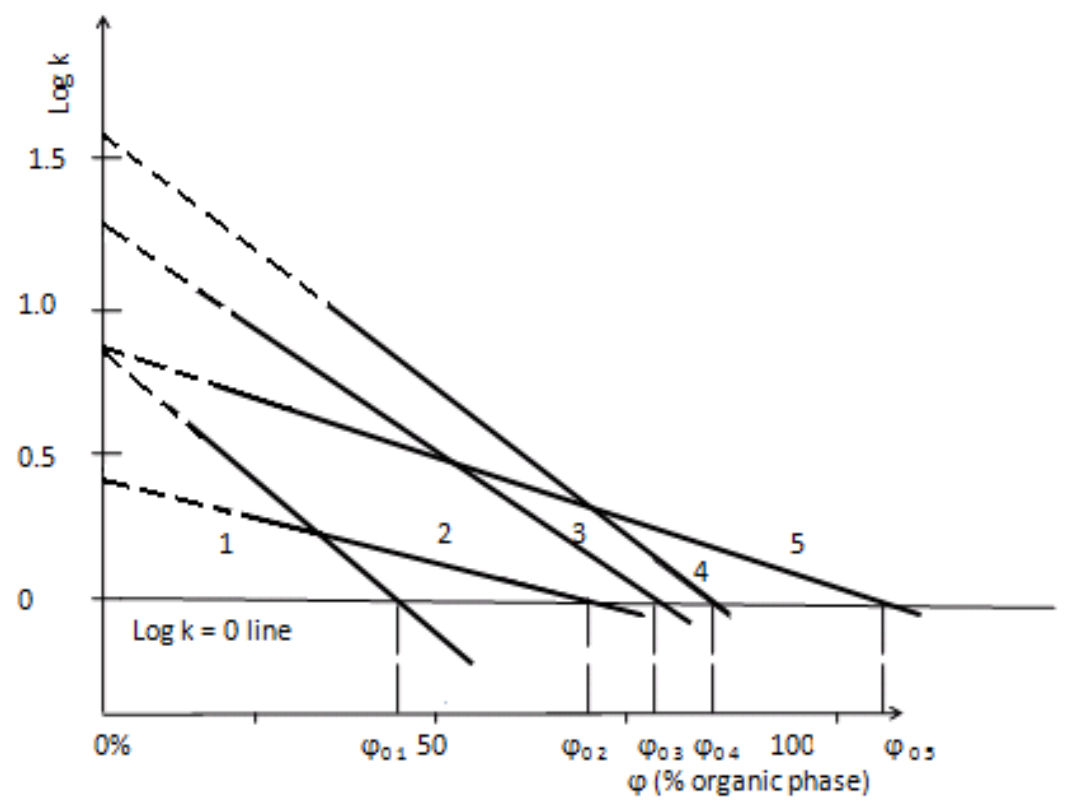

Figure 2.4. The intersection points of the $\log k v s$. $\varphi$ lines for hypothetical compounds.

The $\mathrm{x}$-axis provides the $\varphi_{0}$ values of each compound that is defined as the isocratic chromatographic hydrophobicity index

The quotient $\left(\varphi_{0}\right)$ is equivalent to the organic solvent concentration in the mobile phase that is required to get the compound retention time exactly twice that of the dead time, i.e. $\log k=0$. The $\varphi_{0}$ value of a compound is defined as the chromatographic hydrophobicity index (CHI). It ranges from 0 to $100 \%$ which also indicates an appropriate organic phase concentration by which the compound can be eluted from the column in a reasonably short time (retention time is double that of the dead time). Based on the data for over 500 compounds [31], an approximation can be made for the organic phase concentration in the mobile phase using acetonitrile $(\mathrm{AcN})$ and methanol $(\mathrm{MeOH})$ based on calculated octanol/water partition coefficients $(\log P)$ of a compound using Equations 2.7 and 2.8.

$$
\begin{aligned}
& \varphi_{0}(\mathrm{AcN})=16 \log P+26 \\
& \varphi_{0}(\mathrm{MeOH})=13 \log P+42
\end{aligned}
$$

The advantage of $\varphi_{0}$ is that it does not involve an extrapolation to outside a measurable range as is often required to obtain the $\log k_{\mathrm{w}}$ values. An important aspect of the isocratic $\mathrm{CHI}$ is that it has been derived from the retention data of compounds that were analysed at a mobile phase $\mathrm{pH}$ when the compounds were not ionised and the octanol/water partition coefficients also were obtained for the neutral form of the compounds. The $\mathrm{pH}$ dependence of lipophilicity and the reversed phase chromatographic retention will be discussed later in this chapter. 


\subsubsection{Measurements of lipophilicity using gradient chromatographic retention}

A linear gradient increase of the organic solvent concentration in the mobile phase during a chromatographic run helps to elute compounds over a wide range of lipophilicity. In isocratic mode, several retention time measurements of the same compound are required to establish the $\mathrm{CHI}$, while one measurement in gradient mode can cover compounds with approximately a five order of magnitude difference in partition coefficient. In order to maintain the resolution of the separation, slow organic phase gradient methods are used. Mutton [15] has pointed out that by increasing the flow rate and using short columns it is possible to reduce the gradient time without loss of separation efficiency and resolution. Usually, an acetonitrile gradient is used. The analysis time per compound can be reduced to 5 minutes. During a gradient run any point of the run time is equivalent to a particular organic solvent concentration. By knowing the dead volume and dwell volume of the HPLC system it is possible to estimate the organic phase concentration at the time point when the compound elutes from the column. During a fast organic phase gradient the solvent slope parameter $S$ (see Equation 2.4) has a negligible influence on the gradient retention time. When the appropriate organic phase concentration reaches the column, each compound will travel at approximately the mobile phase velocity. For a first approximation, the retention time in a fast gradient run should be linearly related to the isocratic $\varphi_{0}$ values. It has been shown that the gradient retention times are in good correlation to the isocratically obtained $\varphi_{0}$ values using experimental data from 76 diverse drug molecules obtained from both isocratic and gradient elution [32]. The gradient retention times of a calibration set of compounds give a straight line when plotted against the $\varphi_{0}$ values. The slope and the intercept of the calibration line can be used to convert the gradient retention times to a gradient $\mathrm{CHI}$ that is suitable for inter-laboratory comparison and for building a database [32]. The CHI index measurement takes only 5 minutes using standard HPLC conditions and is a platform widely used by research chemists. The conditions used cover a 5-6 $\log P$ unit range of lipophilicity and simple data processing can be used to convert the gradient retention times to $\mathrm{CHI}$ values. The $\mathrm{CHI}$ values can also be projected to the widely used logarithmic value of the partition coefficient $(\log P)$ by linear conversion. It is important to note that the gradient retention times show a linear relationship to the logarithmic value of the isocratic retention factor. Equation 2.9 shows the $\mathrm{CHI}$ conversion to $\mathrm{CHI} \log D$ while Equation 2.10 shows the CHI conversion to Chrom $\log D$ [33].

$$
\begin{aligned}
& \text { CHI } \log D=0.054 \mathrm{CHI}-1.467 \\
& \text { Chrom } \log D=0.0654 \mathrm{CHI}-2
\end{aligned}
$$

Kern et al. [34] used the gradient retention times of a calibration set of compounds with known $\log D$ octanol/water partition coefficients and converted the gradient retention times directly to octanol/water $\log D$ values. The gradient 
retention times correlated approximately with the $\log D$ values using a Polaris C-18 column and acetonitrile gradient. Donovan and Pescatore [35] have reported the use of gradient methods to obtain a wide range of measured lipophilicity values from gradient retention times, but they use an octadecyl polymer column and a methanol gradient and claim their values to be close to octanol/water partition coefficients. The starting mobile phase $\mathrm{pH}$ is 7.4. The biggest advantage of this methodology is the provision of a lipophilicity parameter directly from the LC-MS compound integrity/purity measurements. In general, using gradient HPLC and C-18 phases, reproducible lipophilicity values can be obtained in a high throughput way while the perfect match with the traditionally used octanol/water partition coefficients is lost. It should be noted that there are significant differences between $\log D$ values derived from gradient retention times and the octanol/water $\log D$ values. For neutral molecules $\mathrm{H}$ bond donor compounds generally show a lower chromatographic log $P$ than the octanol/water $\log P$. Charged molecules however, tend to give higher $\log D$ values in the chromatographic system than octanol/water $\log D$ values (as will be discussed later). So, the conversion is pragmatic and it is used only for expressing the CHI scale (normally 0 to 100 ) as a $\log P$ scale (normally -1 to 5 ). CHI values of more than 200,000 research compounds have been collected at GlaxoSmithKline and proved to be useful in numerous lead optimisation projects. Camurri and Zaramella [36] have adapted a methodology for the MS detection of compounds during the gradient run. The MS detection makes it possible to determine CHI values for mixtures of compounds and in this way further reducing the analysis time. Equivalent selectivity for the fast gradient and the isocratic systems has been demonstrated by Du et al. [37]. The connection between the gradient and isocratic retention times has been discussed extensively by Snyder and utilised in DryLab software [38] that computes isocratic method development conditions based on a few carefully selected gradient measurements.

\subsection{4. pH dependence of lipophilicity and reversed phase chromatographic retention}

Drug molecules often contain ionisable groups which mean that their ionisation state depends on the $\mathrm{pH}$ of the environment. When the compound is ionised the lipophilicity drops dramatically and so does the octanol/water distribution coefficient. Ionised species do not partition into the octanol phase unless a suitable neutral ion-pair is formed. The Henderson-Hasselbalch equation describes the relationship between $\mathrm{pH}$, the acid dissociation constant $\left(\mathrm{p} K_{\mathrm{a}}\right.$ ) and percentage ionisation of compounds that forms the basis of the equation that describes the $\mathrm{pH}$ dependence of distribution coefficients $(\log D)$ as shown by Equations 2.11a and 2.11b for acids and bases, respectively:

$$
\begin{aligned}
& \log D_{(\mathrm{pH})}=\log P-\log \left[1+10^{\left(\mathrm{pH}-\mathrm{p} K_{\mathrm{a}}\right)}\right] \\
& \log D_{(\mathrm{pH})}=\log P-\log \left[1+10^{\left(\mathrm{p} K_{\mathrm{a}}-\mathrm{pH}\right)}\right]
\end{aligned}
$$


Figure 2.5 shows a typical $\log D$ vs. $\mathrm{pH}$ profile for an acid with a $\mathrm{p} K_{\mathrm{a}}=8$, while Figure 2.6 shows the $\log D$ vs. $\mathrm{pH}$ profile for a base with a $\mathrm{p} K_{\mathrm{a}}=8$.

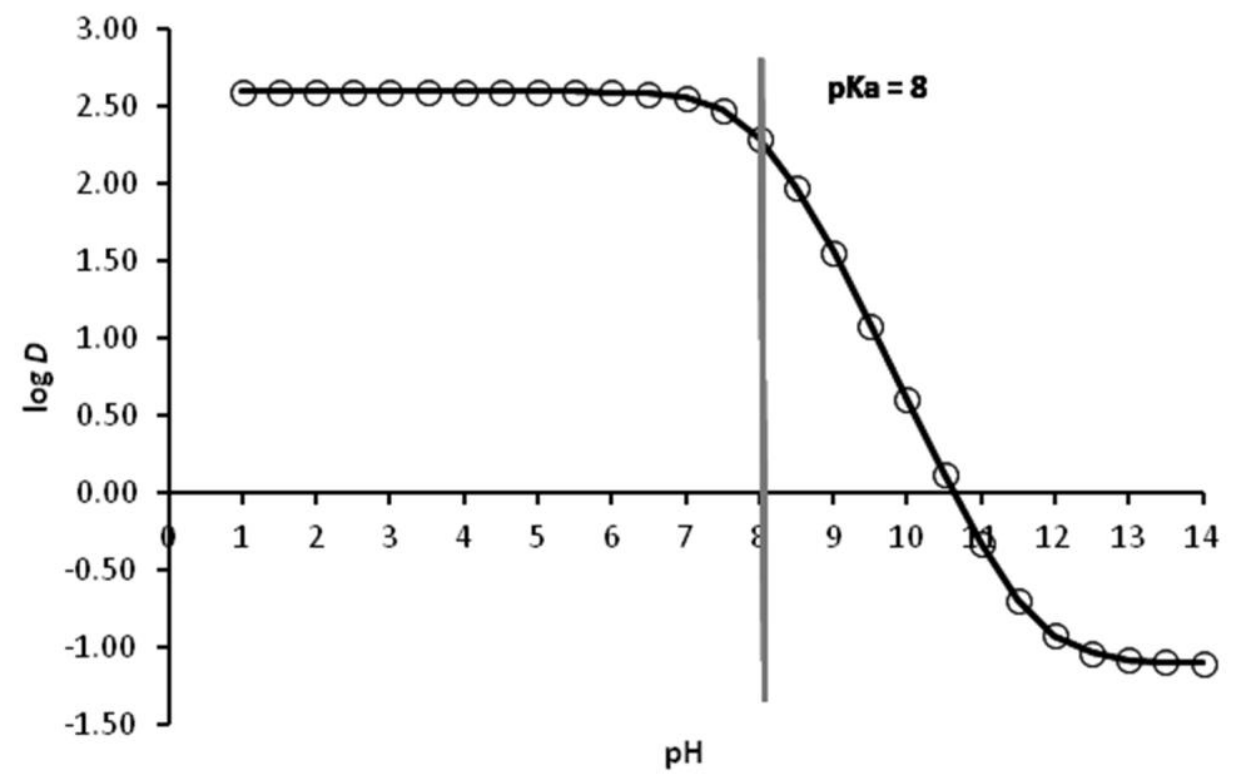

Figure 2.5. Typical $\log D-\mathrm{pH}$ profile for an acid $\left(\mathrm{p} K_{\mathrm{a}}=8\right)$

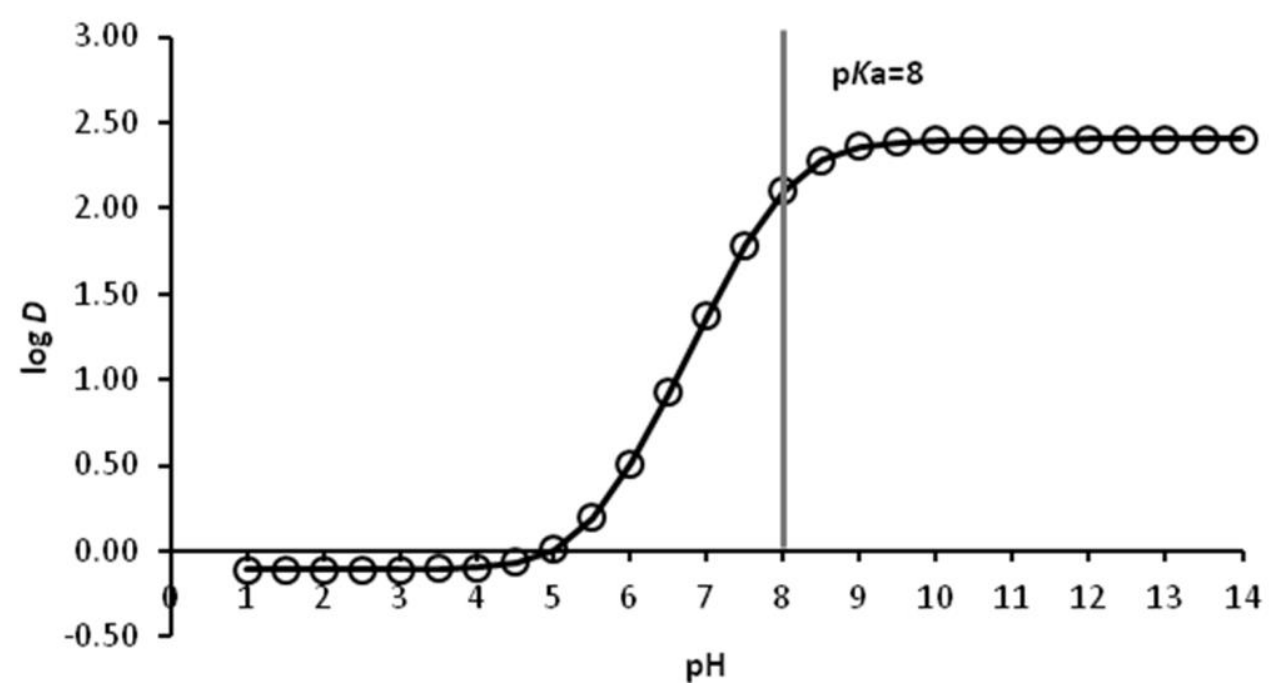

Figure 2.6. Typical $\log D-\mathrm{pH}$ profile for a base $\left(\mathrm{p} K_{\mathrm{a}}=8\right)$

Equations $2.11 \mathrm{a}$ and $2.11 \mathrm{~b}$ do not take into account the partitioning of the fully ionised form of the molecules which is normally 3 to 4 orders of magnitude smaller than the neutral form's partition coefficient. 
Note that when $50 \%$ ionisation occurs (pH 8) the lipophilicity of the compound is only $0.301(\log 2)$ less than the lipophilicity of the neutral form. Canals et al. [39] investigated the gradient retention time $v s$. $\mathrm{pH}$ profile using acetonitrile gradients. The first problem with using acetonitrile gradients is that the mobile phase $\mathrm{pH}$ is influenced by the acetonitrile concentration and therefore it is continuously changing during the analysis. Canals et al. [39] have reported that the $\mathrm{pH} 2$ starting mobile phase can increase up to nearly $\mathrm{pH} 5$ when the acetonitrile concentration reaches $95 \%$. The high $\mathrm{pH}$ starting mobile phase $\mathrm{pH}$ dropped from $\mathrm{pH} 10.5$ to $\mathrm{pH} 8.5$ when the acetonitrile concentration changed from 0 to $95 \%$. Interestingly, the neutral $\mathrm{pH}(\mathrm{pH} 7.4)$ remained stable when an ammonium acetate buffer and acetonitrile gradient was used as shown in Figure 2.7.

However, the compound's $\mathrm{p} K_{\mathrm{a}}$ values are also affected by the presence of the organic phase in the aqueous buffer. Figure 2.8 shows the $\log D$ vs. pH profile of 4-tert-butylpyridine obtained from the gradient CHI expressed as CHI $\log D$, and the $\mathrm{pH}$ profile expected from the octanol/water partition system. The result of the study is that an empirical constant needs to be introduced for weak acids and weak bases into Equations 2.11a and 2.11b to fit the gradient retention time vs. pH profile obtained experimentally as shown by Equation 2.12.

$$
g t_{\mathrm{R}}=\frac{\left(g t_{\mathrm{R}(\mathrm{HA})} 10^{S\left(\mathrm{p} K_{\mathrm{a}}-\mathrm{pH}\right)}+g t_{\mathrm{R}(\mathrm{A})}\right)}{10^{S\left(\mathrm{p} K_{\mathrm{a}}-\mathrm{pH}\right)}+1}
$$

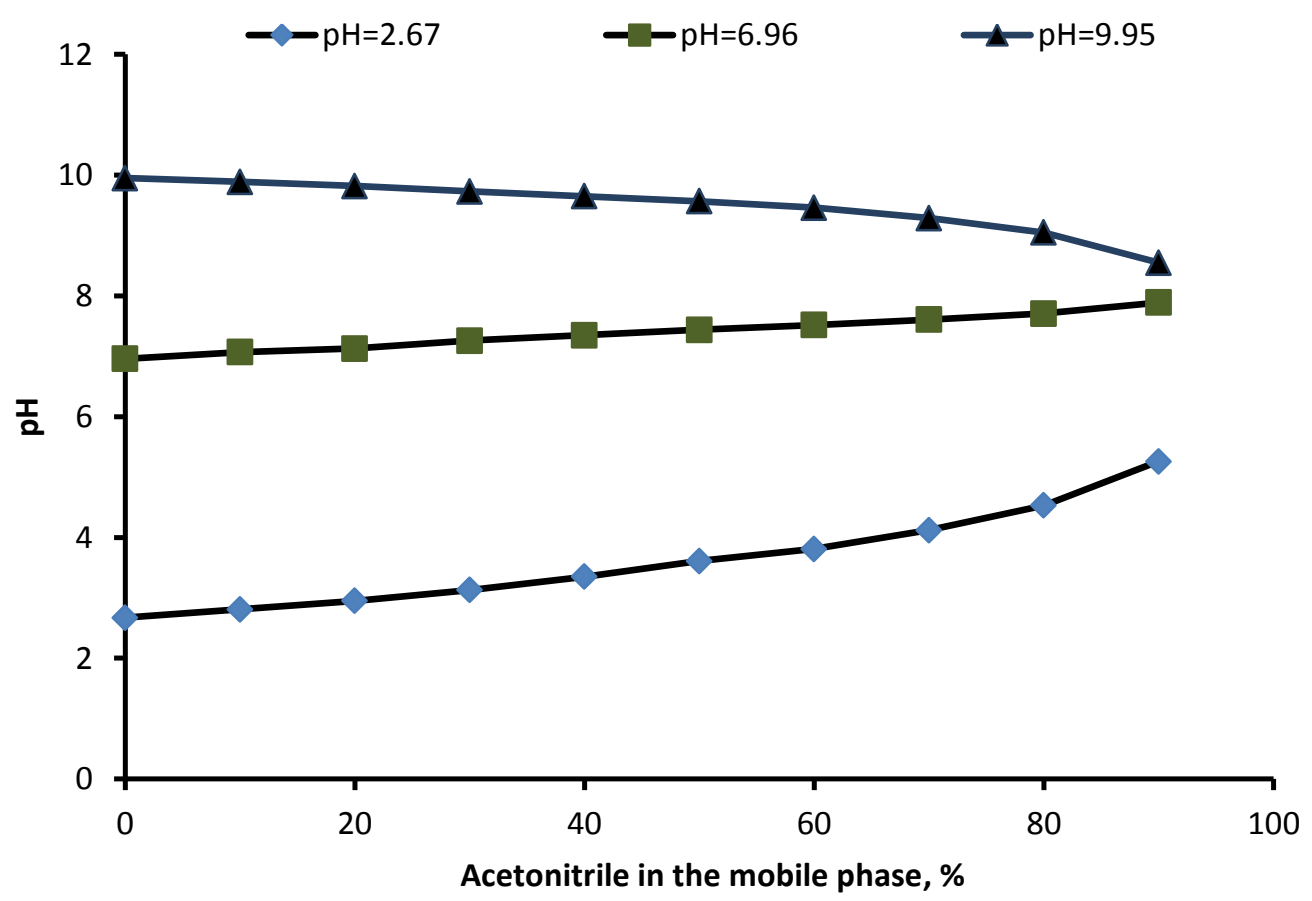

Figure 2.7. The change of the buffer $\mathrm{pH}$ by mixing with various amounts of acetonitrile 
Thus, there is a possibility to measure the gradient retention time, $g t_{\mathrm{R}}$, of a compound using several starting mobile phase pHs and a fitting Equation 2.12 to the $g t_{\mathrm{R}} v s$. pH plot and calculate the compound's $\mathrm{p} K_{\mathrm{a}}$. However, the accuracy of the $\mathrm{p} K_{\mathrm{a}}$ determination depends on the number of experiments, that is, the number of pH's applied for the gradient retention time determination. So, even if we use a fast generic $\mathrm{pH}$ gradient that takes, for example, $4 \mathrm{~min}$, it would take an hour to measure the $\mathrm{p} K_{\mathrm{a}}$ of a compound using 15 experiments at different $\mathrm{pHs}$. In order to have an approximate idea of the compound's acid/base character, in practice we use only three gradient retention time measurements: low $\mathrm{pH}(\mathrm{pH} 2-3)$, physiological $\mathrm{pH}(\mathrm{pH}$ 7.4) and high $\mathrm{pH}$ (pH 9-11). We calculate the CHI value by applying a simple algorithm, and then the compounds are grouped into six categories: acid, base, neutral, weak acid, weak base, and amphoteric (see Figure 2.9).

This simple algorithm is based on the comparison of obtained CHI values at the three different pHs. If the CHI values are the same (within $5 \mathrm{CHI}$ units) at all three pHs, the compound is considered neutral at physiological $\mathrm{pH}$. We have to be careful however, as zwitterionic molecules that are always ionised (either positive or negative or both) could behave as neutral. When the CHI value is significantly greater (more than $5 \mathrm{CHI}$ units) at low $\mathrm{pH}$, but the values are the same (within 5 CHI units) at $\mathrm{pH} 7.4$ and high $\mathrm{pH}$, then the compound is considered an acid, and we can be sure that it contains negatively charged species at physiological $\mathrm{pH}$.

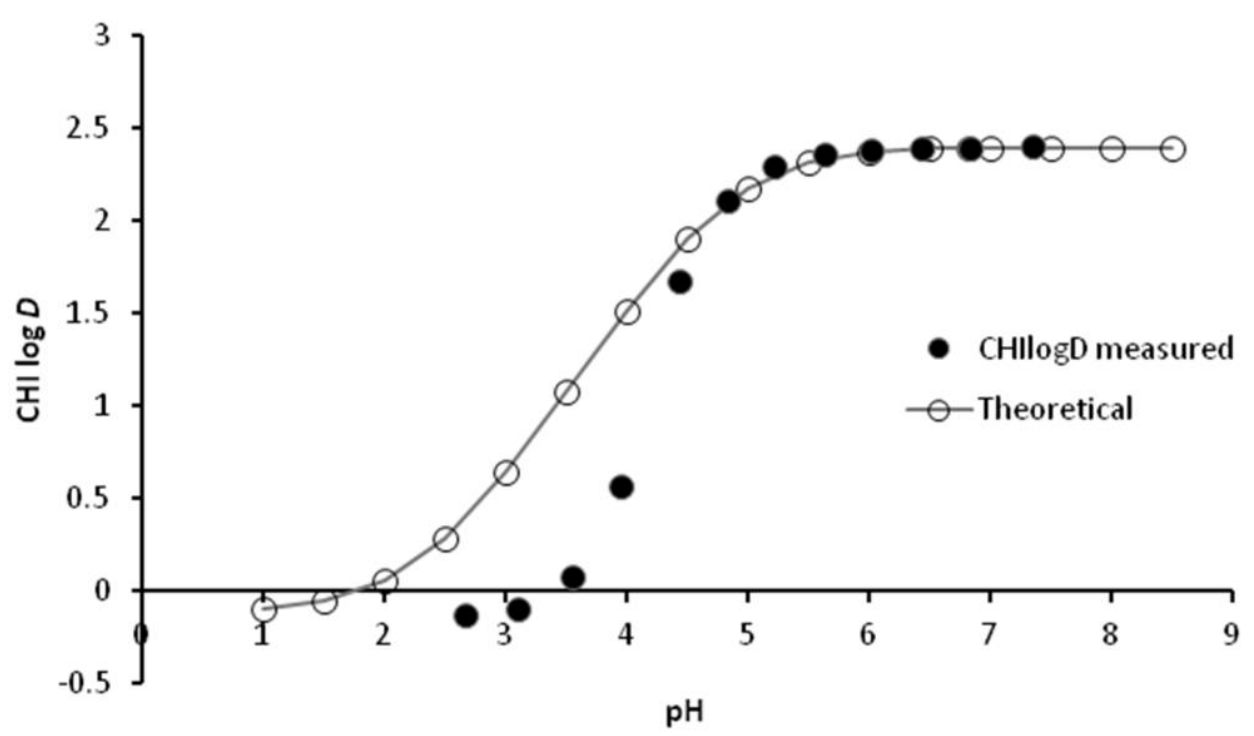

Figure 2.8. The theoretical CHI log D vs. pH profile (open circle by Equation $2.11 \mathrm{~b}$ ) and the experimental CHI $\log D$ vs. pH profile (full circles by Equation 2.12) for a weak base 


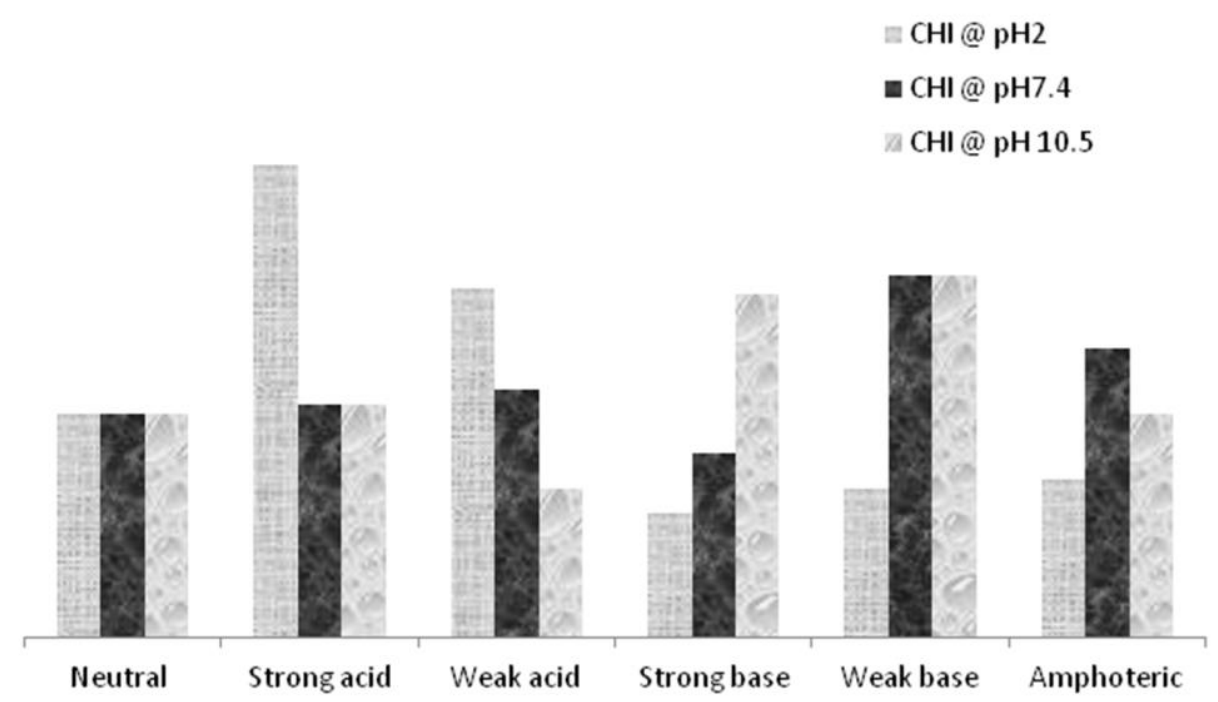

Figure 2.9. Categorising compounds according to their acid/base character using the measurement of Chromatographic Hydrophobicity Index (CHI) at three pHs (acidic, neutral and basic)

When the same $\mathrm{CHI}$ values are obtained at low $\mathrm{pH}$ and $\mathrm{pH} 7.4$, but values are significantly lower (more than $5 \mathrm{CHI}$ units) at basic $\mathrm{pH}$, then the compound is assigned as a weak acid and it can be considered neutral (unionised) at pH 7.4. When a compound has the same $\mathrm{CHI}$ value at $\mathrm{pH} 7.4$ and basic $\mathrm{pH}$, but the $\mathrm{CHI}$ is significantly lower at acidic $\mathrm{pH}$, then the compound is assigned as a weak base, and it is considered as neutral (unionised) at $\mathrm{pH}$ 7.4. Basic compounds show significantly higher $\mathrm{CHI}$ values (more than 5 units) at higher pHs, and they are positively charged at physiological $\mathrm{pH}$.

Another approach has been suggested by Kaliszan et al. $[40,41]$ to determine the acid dissociation constant $\left(\mathrm{p} K_{\mathrm{a}}\right)$ and lipophilicity of compounds using two gradient reversed phase chromatographic measurements. The first measurement should be carried out using a starting mobile phase $\mathrm{pH}$ at which the compound is not ionised (high $\mathrm{pH}$ for basic compounds, low $\mathrm{pH}$ for acidic compounds) and using an organic phase ( $\mathrm{MeOH}$ or AcN) gradient. Based on the compound's retention time in the first experiment, the organic phase concentration that results in a retention factor greater than 10 should be calculated. The second experiment is a $\mathrm{pH}$ gradient that starts at high $\mathrm{pH}$ for basic compounds and reducing to low $\mathrm{pH}$, and starting low $\mathrm{pH}$ for acidic compounds and increasing to high $\mathrm{pH}$. The organic phase concentration should be kept constant. From the retention time of the second experiment the compound's $\mathrm{p} K_{\mathrm{a}}$ can be calculated. 


\subsection{APPLICATION OF BIO-MIMETIC HPLC STATIONARY PHASES}

One of the great advantages of using HPLC technology for measuring properties of compounds is that various types of stationary phases can be applied including ones that mimic biologically important constituents such as membranes and proteins. Protein stationary phases chemically bonded to silica particles have been originally developed for chiral separations [42]. There are two protein bonded commercially available stationary phases that are important not only for chiral separation, which include the human serum albumin (HSA) and alpha-1-acid-glycoprotein (AGP). These two proteins can be found in human and animal plasma. While plasma proteins contain around 40\% HSA, the AGP concentration is much lower $(1-3 \%)$. Although the AGP concentration in plasma is relatively low, it has great significance as it can bind basic (positively charged) drug molecules very strongly, while HSA binds the lipophilic and negatively charged compounds. The major component of the plasma proteins are immunoglobulins which bind compounds based on their lipophilicity regardless of whether they are negatively or positively charged.

\subsubsection{Immobilised human serum albumin (HSA) stationary phase}

Several authors presented good correlations between the logarithmic retention factors measured on the HSA stationary phase and plasma protein binding of drugs obtained by equilibrium dialysis or ultra-filtration methods [43-46]. The principles of using immobilised target bio-polymers to measure drug affinity by HPLC have been reviewed by Bertucci et al. [47]. However, Bucholz et al. [48] reported the lack of correlation between plasma protein binding and HPLC retention measured on HSA columns. There are two important aspects that should be taken into account when comparing the HPLC method with the equilibrium dialysis method for measuring compounds binding to plasma proteins. Firstly, the unit of the binding measure has to be comparable. Plasma protein binding is most often expressed as \% bound or fraction unbound, while in HPLC the logarithmic retention factor measures the partition coefficient of the compound between the aqueous mobile phase and the protein stationary phase. The two measures are in a sigmoid relationship with each other as can be seen in Figure 2.10. As shown, the relatively large changes in log $K$ result in minor changes in \% bound at the high binding region. Both measures can be calculated from one another using Equations 2.13 and 2.14:

$$
\begin{aligned}
& \% \text { bound(HSA })=\frac{k}{k+1} \\
& \log K_{(\mathrm{HSA})}=\log \left(\frac{\% \text { bound }(\mathrm{HSA})}{100-\% \text { bound }(\mathrm{HSA})}\right)-\log [\mathrm{HSA}]
\end{aligned}
$$


where $k$ is the chromatographic retention factor in the HSA stationary phase and $K$ is the binding affinity constant. However, this equation is valid only for nonspecific binding, when compound retention is independent of the injected amount and the overall binding can be modelled by partition. The chromatographic retention factor $k$ is equivalent to the albumin partition coefficient only if the $V_{\mathrm{s}} / V_{\mathrm{m}}$ equals 1 in Equation 2.2.

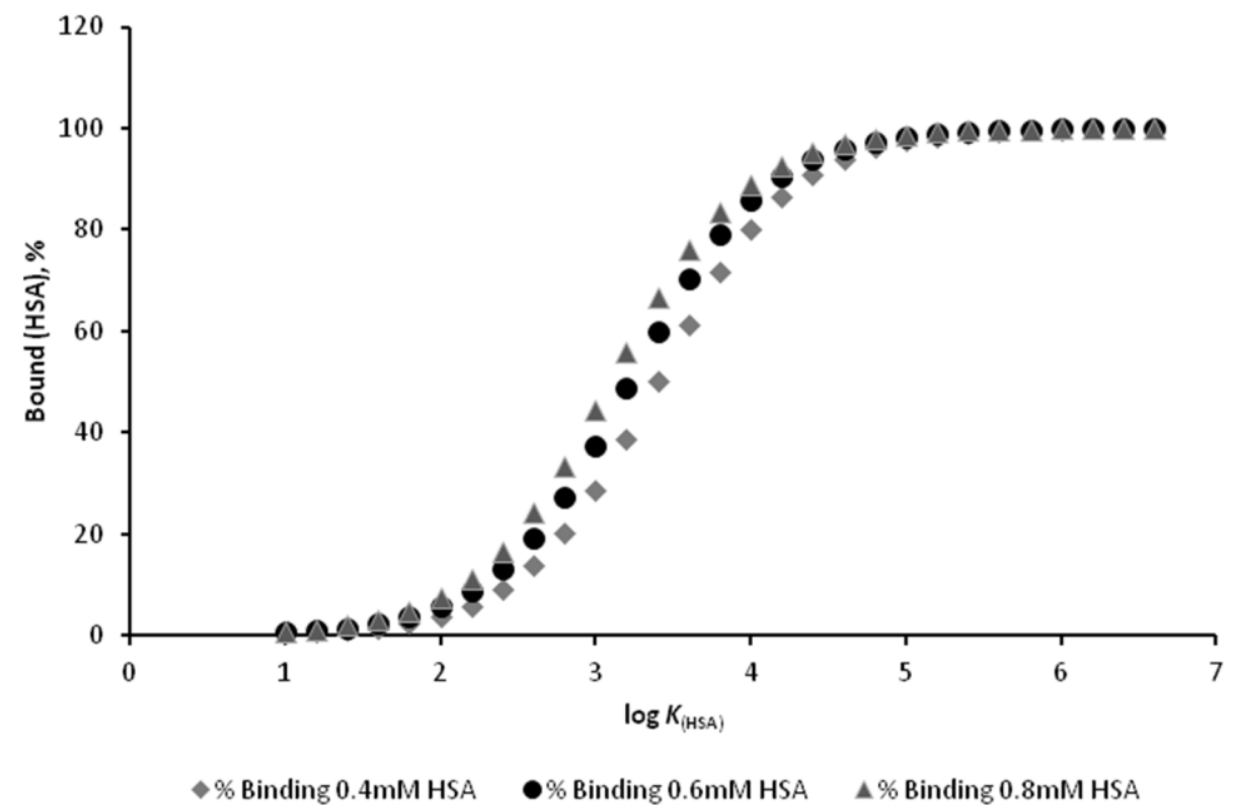

Figure 2.10. The sigmoid relationship between the logarithmic retention factor $(\log k)$ on HSA stationary phase and \% bound HSA

The affinity constant also depends on the HSA concentration, which may vary. Figure 2.10 also shows that the same binding affinity constant may represent a different value when it is expressed as \% bound depending on various physiological (or pathological) concentrations of HSA in the plasma. Secondly, a typical mistake is to compare the value of the albumin binding to the value of plasma protein binding as plasma contains only $40 \% \mathrm{HSA}$, it is very likely that the compound to a certain extent binds to other plasma proteins too. The deviations are most often observed with basic compounds that are positively charged at physiological pHs and bind strongly to AGP, for example. Kratochwil et al. [49] rightfully compared the affinity constants obtained by chromatographic and equilibrium dialysis methods using purified albumin, not plasma. It is also important to keep in mind that the chromatographic method measures the proportion of the average number of moles of the drug in the mobile and the stationary phase during the dynamic equilibrium process. Thus, it measures not only the compounds binding to one particular binding site of the albumin, but many other non-specific binding sites as well. Thus, we cannot assume one to one binding between the albumin and drug molecules. In a methodical point of view it 
is also important to imitate the physiological condition during the chromatographic retention measurements. The best approach is to measure at $\mathrm{pH} 7.4$, the $\mathrm{pH}$ of the plasma, and use isocratic conditions preferably without using an organic modifier in the mobile phase. As it was found by Tiller et al. [44], the $k /(k+1)$ proportion derived from the retention factor multiplied by 100 gave very close values to the reported plasma protein binding \% for a variety of drug molecules even when injected together in a mixture and the chromatographic peaks were identified by MS. Valko et al. [46] applied a fast iso-propanol gradient during the retention time measurements in order to reduce the analysis time for strongly bound compounds. The obtained correlation of the calibrated logarithmic retention time values showed acceptable correlation to the plasma protein binding data in the literature. The good correlation was endorsed by the study of Kratochwil et al. [49] when they compared chromatographic binding data with binding data obtained using only HSA, (and not pooled plasma) and equilibrium dialysis. However, the correlation using data obtained by Colmenarejo et al. [50] who used acetonitrile as an organic modifier in the mobile phase was not as good. This is possibly because the acetonitrile changes the natural three dimensional structure of the albumin and changes its binding characteristics. The practical recommendation of the HSA HPLC columns for chiral separation is to use a small percentage of isopropanol as an organic modifier that supports the hypothesis that under such conditions the naturally chiral selective binding sites are intact. Therefore, it is advisable to use an enantiomeric mixture of warfarin that binds to the major binding site of albumin to check the enantio-selectivity of the stationary phase as it often deteriorates after extensive use of the column.

\subsubsection{Immobilised $\alpha$-1-acid-glycoprotein (AGP) stationary phase}

Similarly to the HSA stationary phase, the AGP stationary phase has been developed for chiral separations. The AGP stationary phase was studied by Jewell et al. [51] who concluded that the retention factors obtained on AGP columns did not show correlation to AGP binding data obtained by in vitro displacement experiments. It is not surprising as the displacement experiments measure compounds binding to a particular binding site of the protein where the probe molecule binds. The chromatographic approach based on retention time measurements indicates the compound's overall partitioning to the protein phase and as such includes specific and non-specific binding. Good correlation has been found between the AGP binding of known drugs obtained by ultra-filtration and chromatographic methods [52] as demonstrated in Figure 2.11.

Some of the positively charged compounds showed stronger binding by the HPLC based measurements than by ultra-filtration. A possible explanation could be that under the ultra-filtration conditions the positively charged compounds saturated the negatively charged specific binding site due to the higher proportion of the drug concentration relative to the protein. 


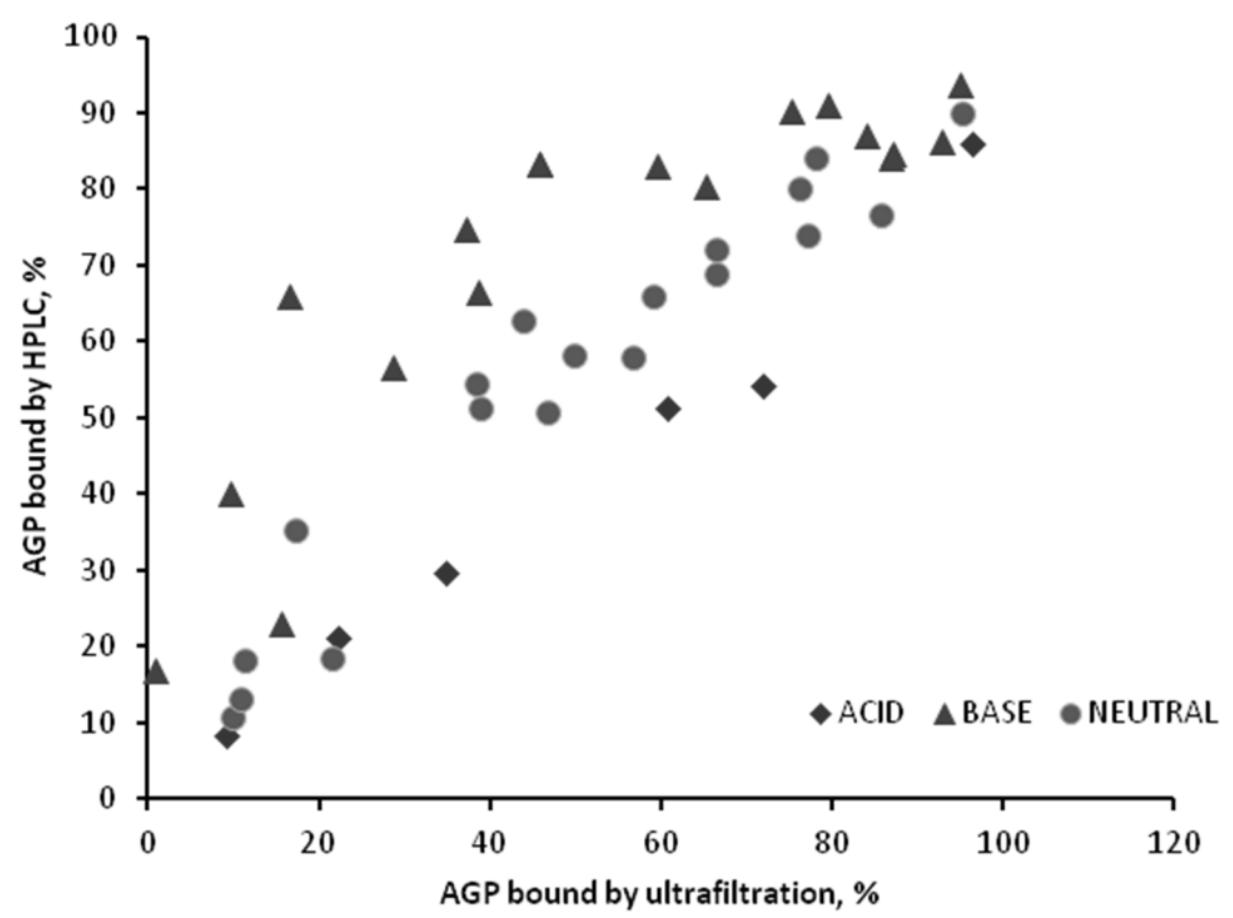

Figure 2.11. The correlation of AGP binding obtained by ultrafiltration method and chromatographic retention time measurements on immobilised AGP HPLC stationary phase. The basic acidic and neutral compounds marked differently

The proportion of the drug concentration to the protein concentration in chromatography is much closer to the physiological condition. Kaliszan et al. [53] studied the binding behaviour of positively charged drugs on an immobilised AGP stationary phase, and proposed a funnel shaped binding site that binds positively charged compounds to the negatively charged narrow end of the funnel. As AGP contains a large proportion of a sialic acid moiety, its presence could explain the strong affinity to basic compounds.

There are other chromatographic methods such as frontal and displacement chromatography that can be used to probe a compound's interactions with proteins as reviewed by Oravcova et al. [54]. For a quick assessment and ranking of compounds binding to plasma proteins, the HPLC based retention time measurements on immobilised protein stationary phases are recommended. It is advisable to apply a calibration set of compounds for which the retention time measurements are frequently repeated in order to check the column performance and suitability, and to increase the day to day and instrument to instrument reproducibility of the data. 


\subsubsection{Immobilised artificial membrane (IAM) stationary phase}

Pidgeon and Venkataram [55] patented a method for immobilising phospholipids on HPLC grade silica stationary phases. The immobilised phospholipids mimic the lipid environment of a fluid cell membrane on a solid matrix (see Figure 2.12 for illustration). Nowadays, various immobilised artificial membrane (IAM) HPLC stationary phases are commercially available. The IAM stationary phases are prepared by covalently bonding the phospholipids to an amino-propyl silica stationary phase. The remaining amino-propyl groups and free silanol groups are treated to reduce their secondary interactions of the compounds. The most frequently used IAM stationary phase contains covalently bonded phosphatidyl choline covering the silica particles at monolayer density. There are excellent reviews about the various commercially available IAM stationary phases and their applications for the determination of compound binding to phospholipids by Taillardat-Bertschinger et al. [56-57] and Giaginis and Tsantili-Kakoulidou [58]. Stewart et al. [59] reviewed the application of immobilised artificial membrane chromatography for modelling drug transport. Usually isocratic methods are suggested, incorporating a very low volume (15\%) of acetonitrile in the mobile phase. Equation 2.2 is suggested for the derivation of membrane partition data from isocratic retention factors. Equation 2.4 can be applied for the extrapolation of retention factors $(\log k)$ obtained with various concentrations of organic modifiers to a $100 \%$ aqueous mobile phase. Taillardat-Bertschinger et al. [57] reviewed the application of IAM chromatography in drug discovery in great detail. A gradient method has been developed and published [60] that applies acetonitrile gradient on IAM HPLC columns and provides a Chromatographic Hydrophobicity Index (CHI IAM). The gradient retention times are calibrated with data obtained from isocratic measurements. The measurements and ranking of the phospholipid binding of drug discovery compounds can be used for characterising and prioritising compounds for progression. These data have been successfully applied in various models to estimate compound in vivo distribution as described later in this chapter.

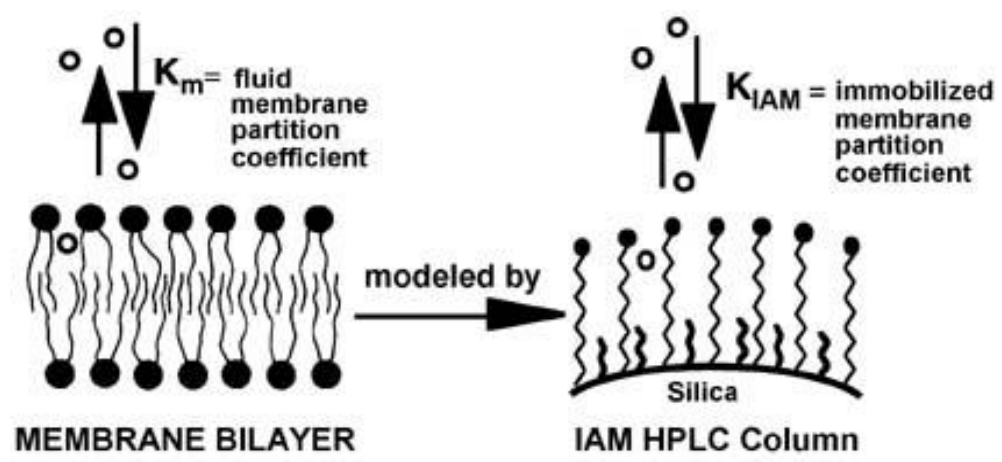

Figure 2.12. The similarity between the IAM stationary phase and a phospholipid bi-layer 


\subsection{GENERAL EXPERIMENTAL DESCRIPTION OF FAST GRADIENT HPLC ANALYSIS OF LIPOPHILICITY, PROTEIN AND PHOSPHOLIPID BINDING OF DRUG DISCOVERY COMPOUNDS}

\subsubsection{Measurements of chromatographic hydrophobicity indices at three different $\mathrm{pHs}$}

The CHI lipophilicity on C-18 stationary phases using three different mobile phases pHs were obtained as described earlier [61]. Typical HPLC conditions are as follows:

HPLC column:

Mobile phase A1 (pH 7.4):

Mobile phase A2 (pH 10.5):

Mobile phase A3 (pH 2):

Mobile phase B:

Flow rate:

Gradient:
Luna C(18)2 $5 \mu \mathrm{m} 50 \times 3 \mathrm{~mm}$ Phenomenex Ltd. UK

$50 \mathrm{mM}$ ammonium acetate $\mathrm{pH} 7.4$

$50 \mathrm{mM}$ ammonium acetate, plus cc ammonia solution to adjust the $\mathrm{pH}$ to 10.5

$0.01 \mathrm{M}$ phosphoric acid acetonitrile (HPLC grade)

$1.0 \mathrm{ml} / \mathrm{min}$

0 to 2.5 min 0 to $100 \% \mathrm{~B}$

2.5 to $3 \min 100 \% \mathrm{~B}$

3.0 to $3.2 \mathrm{~min} 0 \% \mathrm{~B}$

3.2 to $5 \mathrm{~min} 0 \% \mathrm{~B}$

Any HPLC instrument that is able to produce fast gradient analysis, with a lowest possible dwell volume is suitable. UV-detection of the compound can be used when a UV chromophore is available in the analyte. As was highlighted previously, the calibration of the retention time is essential. Any set of compounds that covers a wide range of lipophilicity and chemical diversity can be used for calibration. However, for accurate data that is useful for interlaboratory comparison, the constants for the gradient system should be determined by isocratic measurements. Alternatively one can use the calibration set of compounds presented in Table 2.1 and use the data given for retention time calibration. The gradient retention times of the compounds are converted to $\mathrm{CHI}$ values using the obtained slope and intercept values of the calibration plots. The CHI values can be converted to the octanol/water partition coefficient scale $(\log D)$ using the formula in Equation 2.9. Table 2.1 shows the calibration set of compounds and their CHI values at three different pHs obtained from isocratic measurements with typical gradient retention time data. 
Table 2.1. The calibration set of compounds for the chromatographic hydrophobicity index (CHI) lipophilicity determination at three different pHs [61].

\begin{tabular}{|c|c|c|c|c|c|c|}
\hline Name & $\begin{array}{c}t_{\mathrm{R}} / \mathrm{min} . \\
(\mathrm{pH} 2)\end{array}$ & CHI 2 & $\begin{array}{l}t_{\mathrm{R}} / \min . \\
(\mathrm{pH} 7.4)\end{array}$ & CHI 7.4 & $\begin{array}{c}t_{\mathrm{R}} / \min . \\
(\mathrm{pH} 10.5)\end{array}$ & CHI 10.5 \\
\hline Theophylline & 1.12 & 6.3 & 1.15 & 18.4 & 1 & 4.97 \\
\hline Phenyltetrazole & 1.38 & 17.9 & 1.24 & 23.6 & 1.29 & 15.98 \\
\hline Benzimidazole & 1.78 & 42.2 & 1.41 & 34.3 & 1.58 & 30.61 \\
\hline Colchicine & 1.83 & 43.9 & 1.67 & 42.0 & 1.70 & 43.86 \\
\hline Phenyltheophylline & 1.97 & 51.7 & 1.79 & 51.2 & 1.39 & 21.48 \\
\hline Acetophenone & 2.12 & 64.1 & 1.98 & 65.1 & 2.05 & 64.12 \\
\hline Indole & 2.29 & 72.1 & 2.15 & 71.5 & 2.19 & 72.07 \\
\hline Propiophenone & 2.37 & 77.4 & 2.23 & 77.4 & 2.29 & 77.42 \\
\hline Butyrophenone & 2.55 & 87.3 & 2.43 & 87.5 & 2.46 & 87.33 \\
\hline Valerophenone & 2.72 & 96.4 & 2.60 & 96.2 & 2.61 & 96.36 \\
\hline
\end{tabular}

\subsubsection{Measurement of HSA binding by HPLC retention}

The conditions for generic HSA binding measurements [46] are as follows:

HPLC column: immobilised HSA $50 \times 3 \mathrm{~mm}$ (Chromtech or Chiral Technologies)

Mobile phase A: $50 \mathrm{mM}$ ammonium acetate $\mathrm{pH} 7.4$

Mobile phase B: iso-propanol (HPLC grade)

Flow rate: $\quad 1.8 \mathrm{ml} / \mathrm{min}$

Gradient: $\quad 0$ to $3.0 \mathrm{~min} 0$ to $30 \% \mathrm{~B}$

3.0 to $10.0 \mathrm{~min} 30 \% \mathrm{~B}$

10.0 to $10.5 \min 0 \% \mathrm{~B}$

10.5 to $15.0 \min 0 \% \mathrm{~B}$

Any HPLC instruments that are able to deliver a high flow rate and operate up to 600 bar back pressure can be used. An elevated temperature of up to $40{ }^{\circ} \mathrm{C}$ can be used to reduce the backpressure. Iso-propanol is a viscous solvent and when the $30 \%$ gradient is applied the back pressure can be close to the operational limit of the HPLC instrument. It is possible to apply a lower flow rate, but the gradient slope should be kept the same, and the analysis time should be increased proportionally. UV detection is suitable for UV active compounds. The application of MS detection is very useful, as isomers and enantiomers often produce multiple peaks for which the identification is important. Column performance checks and calibration should be performed before the analysis. The calibration set of compounds can be dissolved separately in a concentration of $0.5 \mathrm{mg} / \mathrm{ml}$ in $50 \%$ iso-propanol and 50\% pH 7.4 ammonium acetate solutions. The calibration set of compounds, their plasma protein binding percentage found in the literature and its linear conversion value (log $K$ lit.), as well as typical retention 
times, their logarithmic values, $\log K$ derived from the calibration curve and \% binding data are listed in Table 2.2. Figure 2.13 shows a typical chromatogram of the warfarin. If the racemates are separated it is considered that the warfarin binding site of the HSA is intact. Figure 2.14 shows a typical calibration plot.

Table 2.2. Calibration set of compounds with their literature and typical measured chromatographic data obtained with the human serum albumin (HSA) column. (Literature data were obtained from reference 46 ).

\begin{tabular}{|lcccccc}
\hline Name & $\begin{array}{c}\text { PPB*, \% } \\
\text { (literature) }\end{array}$ & $\begin{array}{c}\text { Linearised } \\
\text { PPB* \% } \\
(\mathbf{l o g} \boldsymbol{K} \text { lit) }\end{array}$ & $g t_{\mathrm{R}} / \mathbf{m i n}$ & $\begin{array}{c}\mathbf{l o g} \\
\left(\boldsymbol{t}_{\mathrm{R}} / \mathbf{m i n} \text { ) }\right.\end{array}$ & $\begin{array}{c}\mathbf{l o g} \boldsymbol{K} \\
\text { (from } \\
\text { calibration) }\end{array}$ & $\begin{array}{c}\text { HSA } \\
\text { binding, \% } \\
\text { (from } \\
\text { calibration) }\end{array}$ \\
\hline Nizatidine & 35 & -0.28 & 0.937 & -0.03 & -0.25 & 36.27 \\
\hline Bromazepam & 60 & 0.17 & 1.2 & 0.08 & 0.09 & 55.95 \\
\hline Carbamazepine & 75 & 0.46 & 1.63 & 0.21 & 0.52 & 77.66 \\
\hline Budesonide & 88 & 0.83 & 1.837 & 0.26 & 0.69 & 83.85 \\
\hline Nicardipine & 95 & 1.20 & 3.01 & 0.48 & 1.38 & 96.95 \\
\hline Indometacin & 99 & 1.69 & 3.845 & 0.58 & 1.72 & 99.12 \\
\hline Piroxicam & 94.5 & 1.16 & 2.821 & 0.45 & 1.29 & 96.06 \\
\hline Diclofenac & 99.8 & 1.92 & 4.2 & 0.62 & 1.85 & 99.58 \\
\hline Flurbiprofen & 99.96 & 1.98 & 4.3 & 0.63 & 1.88 & 99.68 \\
\hline
\end{tabular}

*Plasma protein binding (PPB)

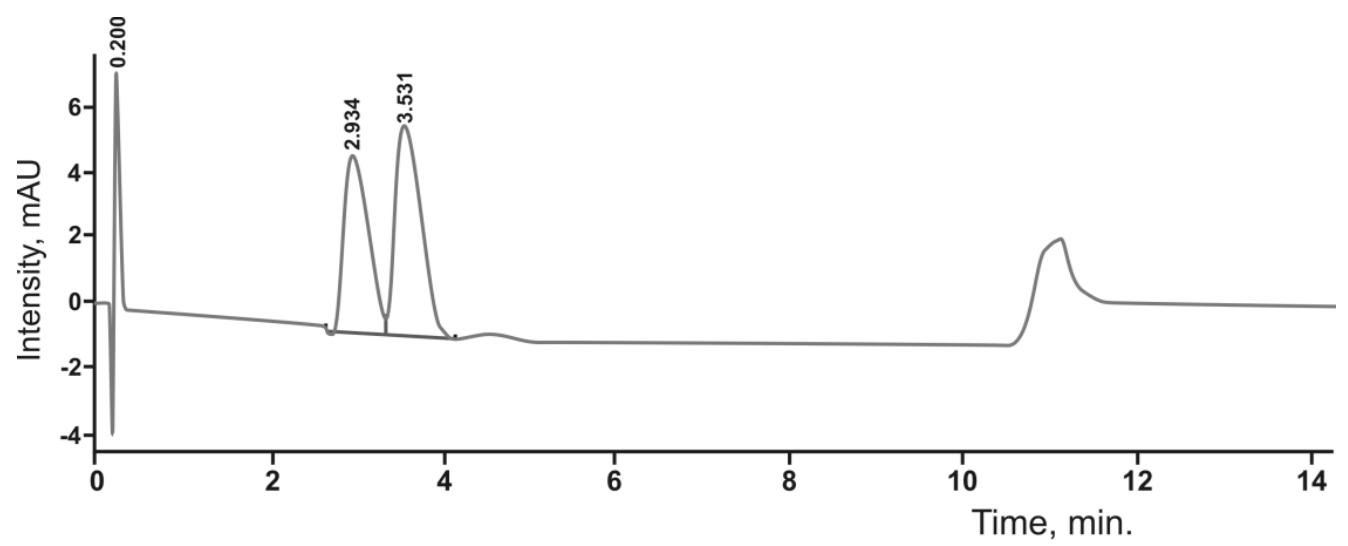

Figure 2.13. Typical chromatogram of racemic mixture of Warfarin. Chromatographic conditions are described in the experimental section 


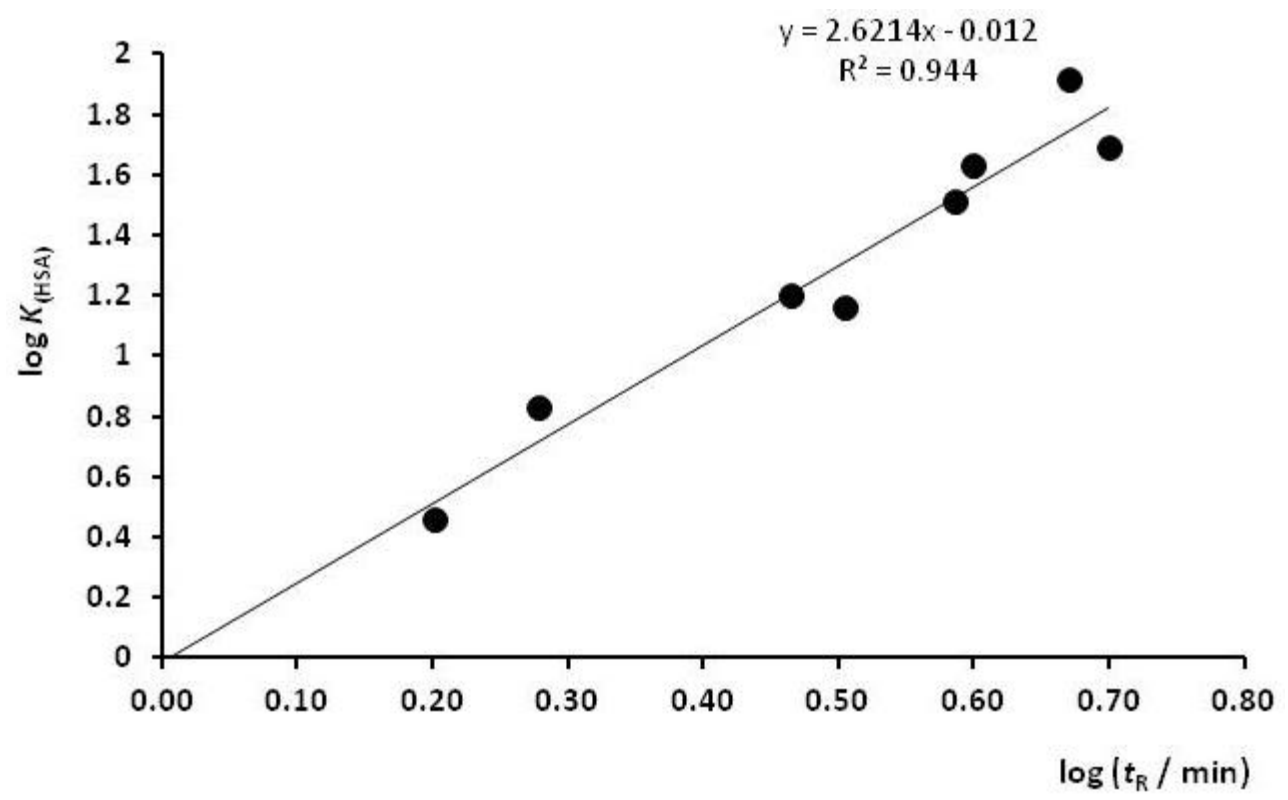

Figure 2.14. Typical calibration plot obtained on the HSA column

The logarithmic value of the gradient retention times $\left(g t_{R}\right)$ obtained from the HPLC experiments were plotted against the linearised values of the percentage of plasma-protein binding, \% PPB (i.e., Log $K$ ). The slope and intercept were then used to convert the gradient retention time values to $\log K$ values for a new compound. From the so obtained log $K$ values, the estimated \% protein binding was obtained by Equation 2.15.

$$
\% \text { bound(HSA })=\frac{101 \times 10^{\log K}}{1+10^{\log K}}
$$

\subsubsection{Measurement of AGP binding by HPLC retention}

The general experimental condition for the AGP binding measurements are as follows:

Column: immobilised AGP $50 \times 3 \mathrm{~mm}$

(Chromtech or Chiral technologies)

Mobile phase A: $50 \mathrm{mM}$ ammonium acetate $\mathrm{pH} 7.4$

Mobile phase B: iso-propanol (HPLC grade);

Flow rate: $\quad 1.8 \mathrm{ml} / \mathrm{min}$

Gradient: $\quad 0$ to 2.5 min 0 to $25 \% \mathrm{~B}$;

2.5 to $4.5 \mathrm{~min} 25 \% \mathrm{~B}$;

4.5 to $4.6 \mathrm{~min} 0 \% \mathrm{~B}$;

4.6 to $6 \operatorname{min~} 0 \% \mathrm{~B}$ 
Instrumentation and detection techniques are the same as described above. Column performance checks and calibration should be performed before the analysis. The compounds used for the column calibrations were dissolved separately in a concentration of $0.5 \mathrm{mg} / \mathrm{ml}$ in $50 \%$ iso-propanol and $50 \% \mathrm{pH} 7.4$ ammonium acetate solutions. The calibration set of compounds, their AGP binding percentage found in the literature and its linear conversion value using Equation 2.1 ( $\log K$ lit.), as well as typical retention times are listed in Table 2.3 with their logarithmic values. Figure 2.15 shows a typical calibration plot.

Table 2.3. The compounds and their literature and experimental alpha-acid-glycoprotein (AGP) binding data.

\begin{tabular}{lcccccc}
\hline Compound & $\begin{array}{c}\text { Binding, \% } \\
\text { (literature) }\end{array}$ & $\begin{array}{c}\text { AGP } \\
\text { (log } \boldsymbol{K} \text { lit) }\end{array}$ & $\begin{array}{c}\text { Exp } \\
\boldsymbol{t}_{\mathrm{R}} / \mathbf{m i n} .\end{array}$ & $\begin{array}{c}\mathbf{l o g} \\
\left(\boldsymbol{t}_{\mathrm{R}} / \mathbf{m i n}\right)\end{array}$ & $\begin{array}{c}\mathbf{l o g} \boldsymbol{K} \\
\text { (from } \\
\text { calibration) }\end{array}$ & $\begin{array}{c}\text { AGP, } \% \\
\text { (form } \\
\text { calibration) }\end{array}$ \\
\hline Nizatidine & 36 & -0.257 & 1.248 & 0.10 & -0.25 & 36.33 \\
\hline Bromazepam & 50 & -0.009 & 1.601 & 0.20 & 0.03 & 52.23 \\
\hline Warfarin & 88 & 0.831 & 2.877 & 0.46 & 0.76 & 86.01 \\
\hline Propranolol & 86 & 0.758 & 2.997 & 0.48 & 0.81 & 87.59 \\
\hline Imipramine & 91 & 0.959 & 3.167 & 0.50 & 0.87 & 89.02 \\
\hline Nicardipine & 92 & 1.010 & 3.349 & 0.52 & 0.93 & 90.32 \\
\hline Chlorpromazine & 92 & 1.010 & 3.975 & 0.60 & 1.15 & 94.34 \\
\hline
\end{tabular}

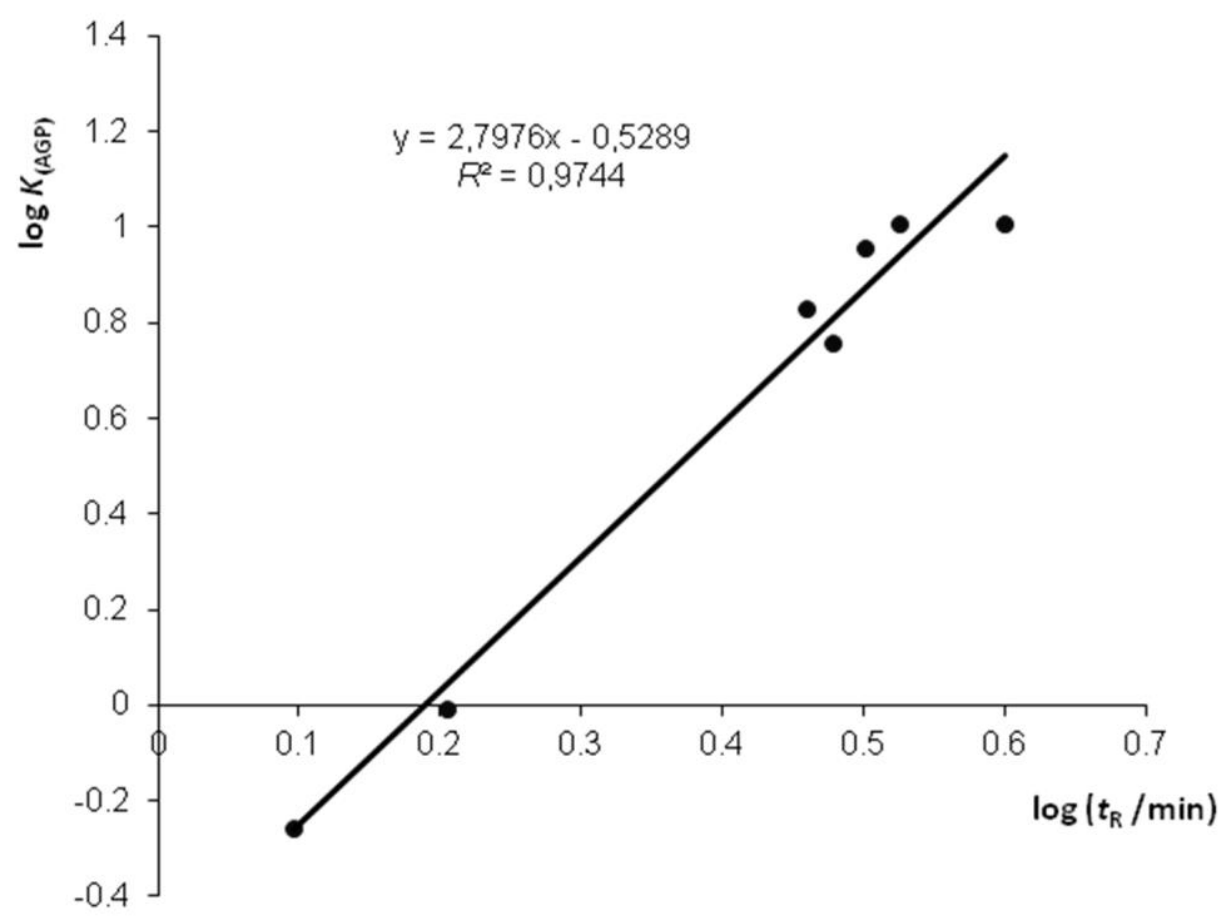

Figure 2.15. Typical calibration plot for AGP binding measurements 


\subsubsection{Measurement of IAM binding by HPLC retention}

The following conditions can be applied for the phospholipid binding measurements [60]:

Column: $\quad$ immobilised artificial membrane column

(Regis Analytical LTD) $150 \times 4.6 \mathrm{~mm}$

Mobile phase A: $50 \mathrm{mM}$ ammonium acetate $\mathrm{pH}$ 7.4;

Mobile phase B: acetonitrile (HPLC grade);

Flow rate: $\quad 2.0 \mathrm{ml} / \mathrm{min}$

Gradient: $\quad 0$ to 2.5 min 0 to $70 \% \mathrm{~B}$

2.5 to $3 \mathrm{~min} 70 \% \mathrm{~B}$

3.0 to $3.2 \min 0 \% \mathrm{~B}$

3.2 to $5 \min 0 \% \mathrm{~B}$

Instrumentation and detection as described above. The column performance check and calibration should be performed before the analysis. The compounds used for the column calibrations are dissolved separately in a concentration of $0.5 \mathrm{mg} / \mathrm{ml}$ in $50 \%$ acetonitrile and $50 \% \mathrm{pH} 7.4$ ammonium acetate solution mixtures. The CHI (IAM) values for the calibration set of compounds have been measured using the isocratic mode [60] and are listed in Table 2.4. A typical calibration plot is shown in Figure 2.16. The CHI (IAM) values were converted to the traditional lipophilicity scale (log $k$ IAM) using the formula below (Equation 2.16) which was obtained by plotting the isocratic log $k$ values against the CHI (IAM) values [60].

$$
\log k(\mathrm{IAM})=0.046 \times \mathrm{CHI}(\mathrm{IAM})+0.42
$$

The constants in Equation 2.16 were obtained by plotting the CHI values obtained from the gradient retention times against the $\log k$ values that were obtained from isocratic retention time measurements and extrapolated to the $100 \%$ aqueous mobile phase.

Table 2.4. The calibration set of compounds and their literature CHI (IAM) values [60]

\begin{tabular}{cccc}
\hline Compound & $\begin{array}{c}\text { typical retention time } \\
\boldsymbol{t}_{\mathbf{R}} / \mathbf{m i n} .\end{array}$ & CHI (IAM) & Log $\boldsymbol{k}$ (IAM) \\
\hline Octanophenone & 3.18 & 49.4 & 2.69 \\
\hline Heptanophenone & 3.07 & 45.7 & 2.52 \\
\hline Hexanophenone & 2.94 & 41.8 & 2.34 \\
\hline Valerophenone & 2.79 & 37.3 & 2.14 \\
\hline Butyrophenone & 2.58 & 32 & 1.89 \\
\hline Propiophenone & 2.35 & 25.9 & 1.61 \\
\hline Acetophenone & 2.04 & 17.2 & 1.21 \\
\hline Acetanilide & 1.85 & 11.5 & 0.95 \\
\hline Paracetamol & 1.62 & 2.9 & 0.55 \\
\hline
\end{tabular}




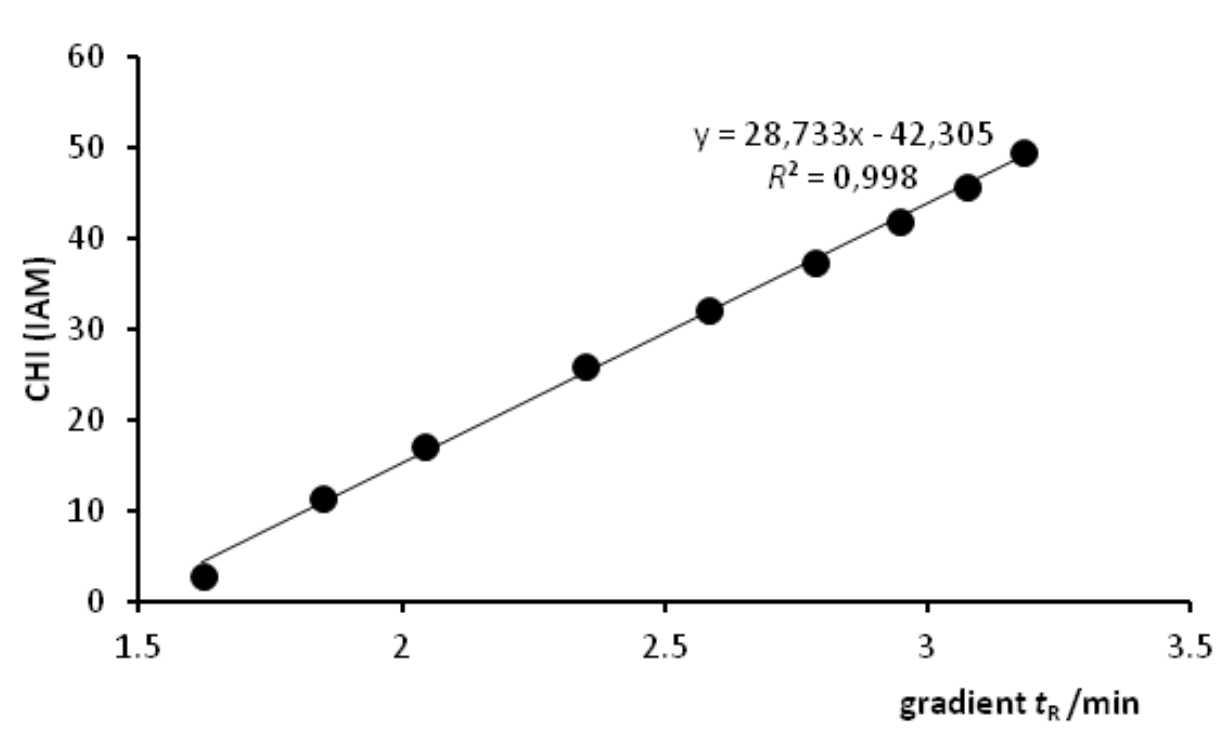

Figure 2.16. Typical calibration curve for the IAM measurements

\subsection{ANALYSIS OF THE HPLC BASED LIPOPHILICITY, PROTEIN BINDING AND PHOSPHOLIPID BINDING DATA}

\subsubsection{Analysis of the effect of charge}

As described above, chromatographic techniques provide a measurement of a compound's interactions with a variety of stationary phases including proteins and phospholipids. It is important to investigate the relationships between these properties. Are we just measuring a compound's lipophilicity in a different way? We expect a minimum binding of a compound to each of the investigated stationary phases based on its hydrophobicity. However, the compound can bind even stronger to a particular stationary phase if it has additional interaction to it above the hydrophobic interactions. In order to detect the additional interactions we can use the octanol/water partition coefficients for comparison. From the seminal work of Hansch et al. [62], the octanol/water partition system is used to model a variety of biological partition processes; therefore it is important to understand the relationships between the bio-mimetic properties and other lipophilicity measures. Figures 2.17-2.18 show plots of HSA and IAM binding values as a function of $\mathrm{CHI} \log D$ values using the data obtained for 70 known diverse drug molecules. Figure 2.19 shows the plot of the HSA binding as a function of IAM binding. We can see in Figure 2.17 that the more lipophilic compounds bind stronger to HSA, however there are several compounds that show even stronger binding than would be expected from their CHI $\log D$ values, especially compounds that are assigned as acid. 


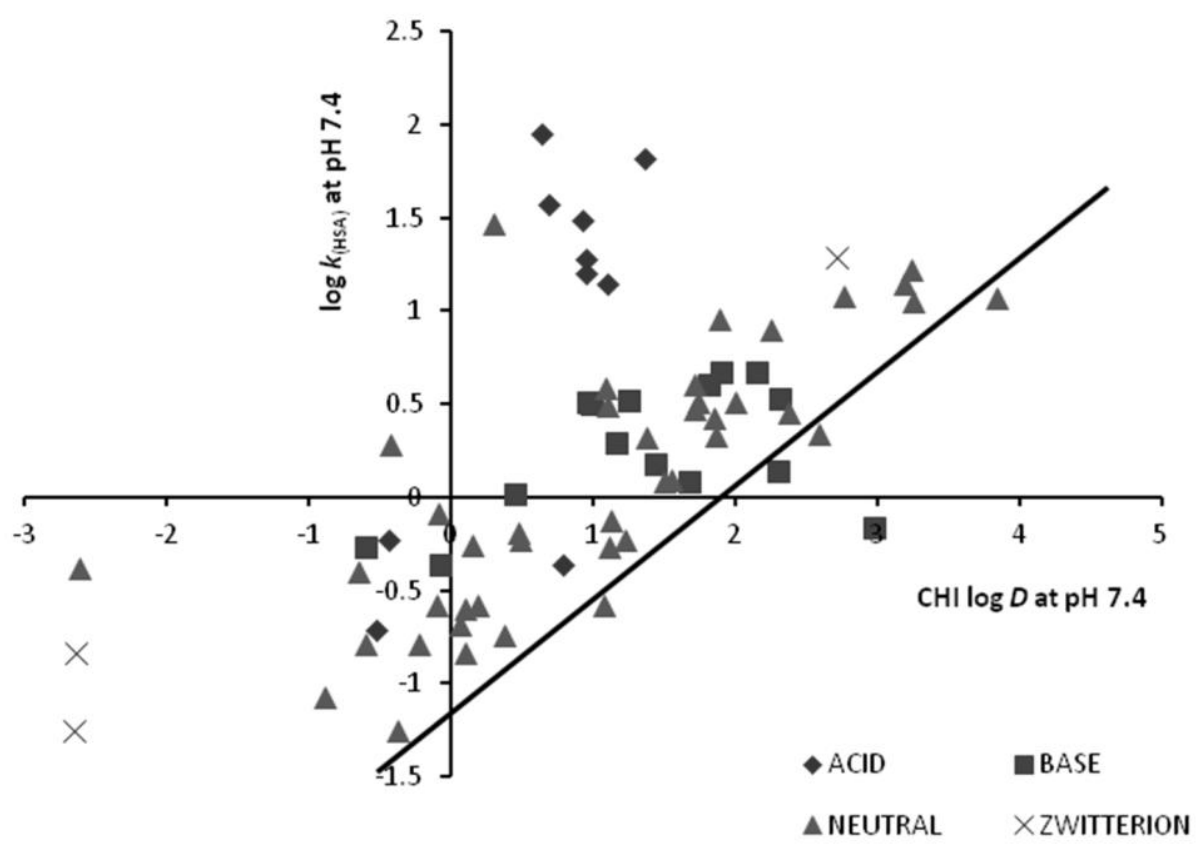

Figure 2.17. Typical plot of the HSA binding values against the $\mathrm{CHI} \log D$ values for known drugs. Acidic, basic and neutral compounds marked differently. The line represents the expected minimum HSA binding based on the lipophilicity ( $\mathrm{CHI} \log D$ )

In Figure 2.18 similar trends can be observed for IAM binding. Compounds with a certain degree of lipophilicity (CHI log D) have an expected binding to IAM. However, many drug molecules show stronger binding to IAM than would be expected from the $\mathrm{CHI} \log D$ values, especially bases. It is interesting to see the plot of HSA and IAM binding in Figure 2.19. It can be seen that the negatively charged compounds bind more strongly to HSA than IAM, while the positively charged compounds bind more strongly to IAM than HSA. We can therefore conclude that the presence of charge causes significant differences between a simple C-18 lipophilicity and IAM and HSA binding.

From these results, it is obvious that the octanol/water $\log D$ or $\mathrm{CHI} \log D$ does not distinguish between the positive or negative charges. Whichever charge is present on the molecule it will reduce its ability to partition into the octanol or C18 stationary phase. However, compounds with a positive charge bind strongly to phospholipids while the negatively charged compounds bind strongly to albumin types of proteins. 


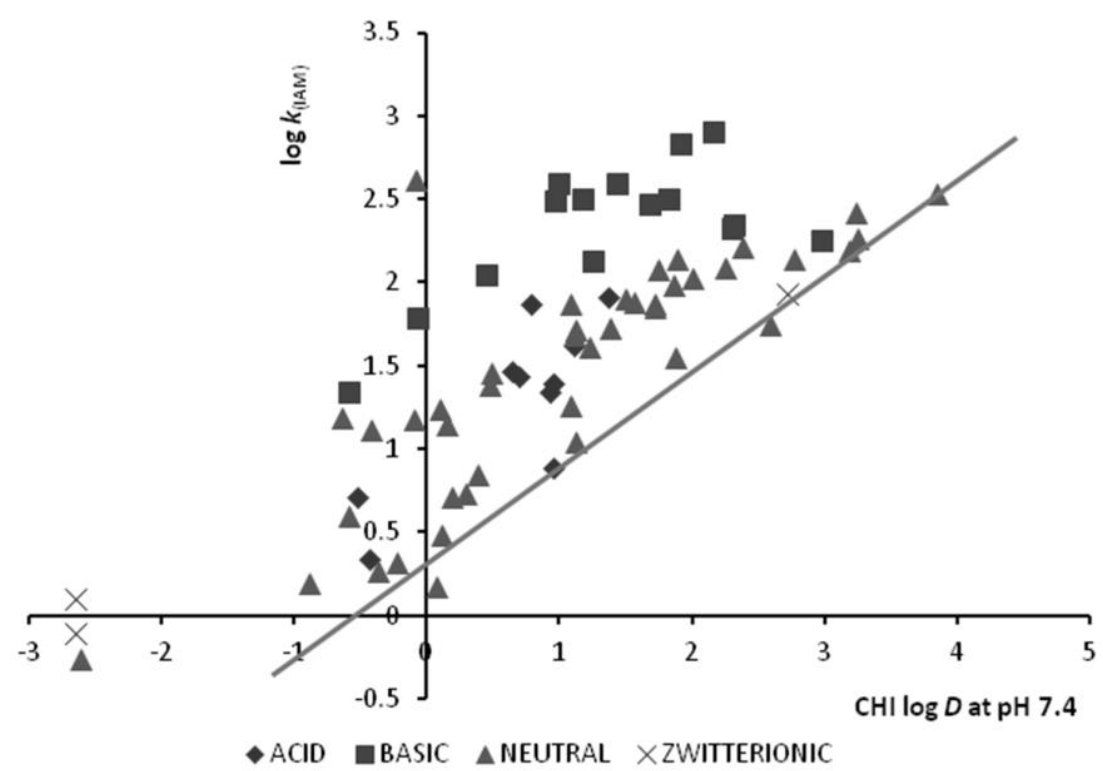

Figure 2.18. The plot of the IAM binding values and the CHI $\log D$ values for known drug molecules. Acidic, basic and neutral compounds marked differently. The line represents the expected minimum IAM binding based on the lipophilicity ( $\mathrm{CHI} \log D$ ). It can be seen that negatively charged acids have lower than the expected minimum binding to IAM

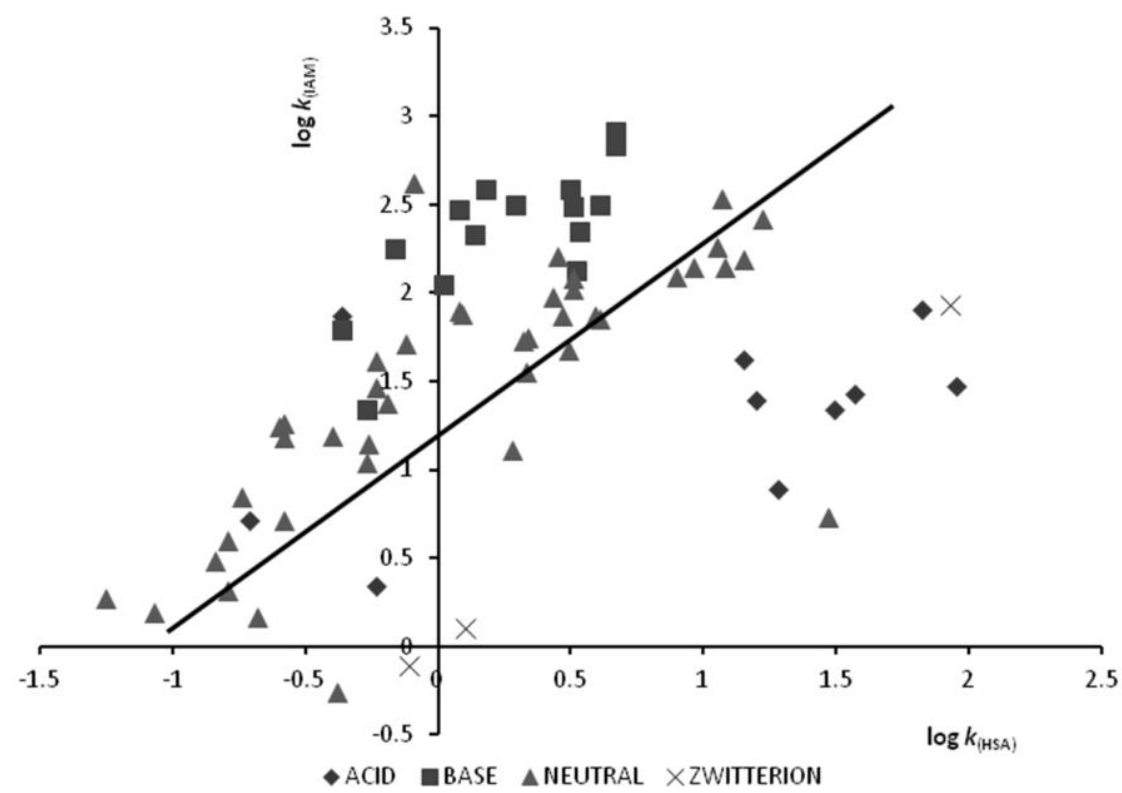

Figure 2.19. The plot of HSA and IAM binding of known drug molecules. Acidic, basic and neutral compounds marked differently. The line represents the expected equivalent binding to IAM and HSA based on compound lipophilicity only, without additional binding interactions 


\subsubsection{Analysis by the solvation equation}

It is worth investigating the similarities or differences of the binding of neutral compounds to albumin and phospholipids and comparing them to the octanol/water partition coefficients. The solvation equation approach can provide a tool for investigating the behaviours of neutral molecules in various water/organic phase partition systems.

The Abraham solvation equation model [63] suggests that molecular size, and a small number of polarity descriptors (H-bond donor/acceptor, dipolarity) are sufficient to describe compound partition behaviour. By measuring $\log k$ (or the extrapolated $\log k_{\mathrm{w}}$ ) values for a set of probe molecules (minimum 25 compounds, preferably 40 - 60) with known molecular descriptors, such as excess molar refraction, $E$; dipolarity/polarisability, $S$; H-bond acidity, $A$; H-bond basicity, $B$; and molecular size, $V$, the solvation equation characteristic for the chromatographic partitioning system can be constructed using the multiple linear regression equation as shown below:

$$
\log k=e E+s S+a A+b B+v V
$$

The regression coefficients of the molecular descriptors (e, $s, a, b$, and $v$ ) are characteristics for the chromatographic partition system. Numerous equations have been published [63-68] for the characterisation of the various reversed phase of columns (C-18, perfluorinated hydrocarbons, cyclodextrin) using acetonitrile and methanol as an organic modifier in the mobile phase. The generally good statistical fit of the chromatographic retention data and the molecular descriptors derived from bulk organic solvent/water partition coefficients support the comparability of the two types of partition data. However, it should be noted, that the parameters of the solvation equation are often based on the retention data of relatively small, mono- or bi-functional molecules in unionised form. Du et al. [69] compared the linear solvation equations obtained for isocratic HPLC retention factors (using C-18 stationary phases and acetonitrile as an organic modifier in the mobile phase) and the octanol water partition coefficients. The major difference between the two partition systems is their sensitivity towards the H-bond acidity of the molecules. While the water saturated octanol phase easily accommodates compounds with H-bond donor groups, the chromatographic C-18 stationary phase has no such polar functionality. Therefore, $\mathrm{H}$-bond donor functional groups decrease chromatographic retention and, consequently, chromatographic partition coefficients. The correlation between isocratic retention data (with C-18 stationary phases and aqueous acetonitrile mobile phases) and octanol $\log P$ can be significantly improved by taking into account a simple H-bond donor count (HBC) or the Abraham H-bond acidity descriptor $(A)$ in the equations shown below:

$$
\begin{aligned}
& \log P_{\text {oct }}=1.91 \log k+0.37 \mathrm{HBC}+0.72(n=111 ; r=0.962 ; s d=0.272) \\
& \log P_{\text {oct }}=2.07 \log k+1.09 A+0.52(n=111 ; \mathrm{r}=0.982 ; \mathrm{sd}=0.189)
\end{aligned}
$$


The gradient chromatographic $\mathrm{CHI}$ values obtained for the unionised forms of the molecules (CHIN) have also shown an acceptable correlation with the octanol/water $\log P$ values when HBC or $A$ are included [61]. Equations 2.20 and 2.21 show the relationships obtained for a training set of 86 known drug molecules.

$$
\begin{aligned}
& \log P_{\text {oct }}=0.047 \mathrm{CHIN}+0.36 \mathrm{HBC}-1.10 \\
& (n=86 ; r=0.943 ; s=0.39 ; F=336) \\
& \log P_{\text {oct }}=0.054 \mathrm{CHIN}+1.32 A-1.88 \\
& (n=86 ; r=0.970 ; s=0.29 ; F=655)
\end{aligned}
$$

Solvation equations have been derived for various biological partition/distribution processes, such as blood/brain barrier distribution [70], oral absorption [71], and skin-penetration [72]. The solvation equations obtained for biological partition processes can be compared with the solvation equations obtained for organic solvent/water partition coefficients [73,74]. In order to compare the various partition systems, the coefficients of each molecular descriptor was divided by the volume coefficient $(v)$, otherwise the scale or unit of the solute parameter distorts the proper comparison of the systems in terms of their sensitivity towards the molecular descriptors. Table 2.5 shows the relative coefficients of the molecular descriptors obtained for various biological, biomimetic, and organic solvent/water partitions.

Table 2.5. The relative coefficients of the molecular descriptors (E, S, A and B) for various biological, bio-mimetic and organic solvent/water partition systems.

\begin{tabular}{|l|l|l|l|l|}
\hline System & \multicolumn{1}{|c|}{$\boldsymbol{e} / \boldsymbol{v}$} & \multicolumn{1}{c|}{$\boldsymbol{s} / \boldsymbol{v}$} & \multicolumn{1}{c|}{$\boldsymbol{b} / \boldsymbol{v}$} \\
\hline $\log k_{\mathrm{IAM}}$ & 0.37 & -0.24 & 0.03 & -1.10 \\
\hline $\mathrm{CHI}_{\mathrm{IAM}}$ & 0.42 & -0.25 & 0.07 & -1.08 \\
\hline $\mathrm{CHI}_{\mathrm{RP}, \mathrm{AcN}}$ & 0.09 & -0.24 & -0.30 & -0.98 \\
\hline $\log P_{\text {octanol }}$ & 0.15 & -0.28 & 0.01 & -0.91 \\
\hline Blood/brain & 0.19 & -0.69 & -0.72 & -1.28 \\
\hline HSA/buffer & 0.02 & -0.07 & 0.16 & -1.21 \\
\hline AGP/buffer & 0.46 & -0.38 & -0.33 & -0.85 \\
\hline water/skin & 0.00 & -0.33 & -0.35 & -1.95 \\
\hline
\end{tabular}

As we can see in Table 2.5, the biggest variation of the coefficients can be observed in the relative sensitivity of the systems towards the H-bond acidity of the compounds. The octanol/water system, the IAM and HSA binding are not sensitive to the H-bond acidity of the molecules. This means that molecules with H-bond donor groups easily partition into octanol and bind to the phospholipid membrane and albumin types of proteins. This can be explained by the fact that 
these systems contain water or another H-bond acceptor moiety that accommodates the H-bond donor groups forming hydrogen bonding. These findings support the idea that the octanol/water partition system is a good model for biologically relevant partitioning of the compounds. However, we can see from Table 2.5 that compound distribution between the blood and brain is strongly affected by the $\mathrm{H}$-bond donor groups and that the $a / v$ coefficient has a negative value. This means that molecules with $\mathrm{H}$-bond donor groups prefer the blood to the brain when partitioning between them. The reversed phase chromatographic system and the water/skin partitioning systems also have a negative coefficient for the H-bond acidity, but slightly less so than the blood/brain system. All systems have very similar coefficients towards the H-bond basicity of the molecules (large negative values), which means the compounds with H-bond acceptor groups prefer the more aqueous environments. We can observe reasonably large variations of the system's sensitivity towards the dipolarity and polarisability parameter $(s / v)$ and the excess molar refraction $(e / v)$, which indicate the differences in polarity (dipole moments) and heteroatom interactions.

The solvation equation approach is a valuable tool for comparing the similarities and differences between physico-chemical partitioning and biological partitioning of the molecules; moreover it can be used to select the best physico-chemical model systems for biological partitioning [75]. We have to remember that the above mentioned solvation equations do not incorporate the effect of ionisation of the molecules and only the data of the uncharged molecules should be used for generating these equations for system comparisons. Recent efforts have been published [76] that estimate the effect of the ionisation on the partitioning of the molecule.

We can conclude from these findings that the physicochemical water/organic solvent partition systems, and the bio-mimetic chromatographic partitioning systems can be used as simple measurements of compound behaviour that are able to imitate compound partitioning in a biological environment. However, it would be even more useful to use these measured parameters in models that describe compound behaviour in vivo.

\subsection{APPLICATIONS OF BIO-MIMETIC HPLC PROPERTIES TO MODEL DRUGS IN VIVO DISTRIBUTION}

There are several experimental approaches that are used to characterise the in vivo distribution of drug molecules and describe their pharmacokinetic and pharmacodynamic behaviour. The body can be divided into several compartments to which the drug molecules are distributed. The simplest model divides the body into two compartments, the plasma compartment and tissue compartment. The volume of distribution, $V_{\mathrm{d}}$, is used as a hypothetical volume that reveals how extensive the compound distribution is into tissues from the plasma. It can be defined in two different ways and can be obtained from the given intravenous dose and the plasma concentration, $C_{\text {plasma, }}$ as described in Equation 2.22. 


$$
V_{\mathrm{d}}=\frac{\text { Dose }}{C_{\text {plasma }}}
$$

As the plasma concentration is continuously changing with time as the compound distributes and then starts to eliminate, the so-called steady state volume of distribution, $V_{\mathrm{dss}}$ is introduced, which considers a repeated dose that results in a steady state plasma concentration. Using the steady state volume of distribution and knowing the volume of the plasma compartment, $V_{\mathrm{p}}$, we can rearrange Equation 2.22 to form Equation 2.23.

$$
V_{\mathrm{dss}}=\frac{\text { Dose } \times V_{\mathrm{p}}}{A_{\mathrm{p}}}
$$

where $A_{\mathrm{p}}$ is the amount of drug in the plasma compartment. We can define an

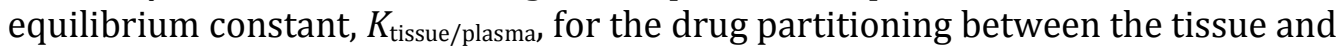
the plasma compartment as the tissue concentration divided by the plasma concentration, supposing that the drug distribution between these compartments are independent from the dose as shown by Equation 2.24.

$$
K_{\text {tissue/plasma }}=\frac{\frac{\text { Dose }-A_{\mathrm{p}}}{V_{\mathrm{t}}}}{\frac{A_{\mathrm{p}}}{V_{\mathrm{p}}}}
$$

$V_{\mathrm{t}}$ is the volume of the tissue compartment. Theoretically, the amount of drug in the tissue compartment is the difference between the dose and the amount of drug in the plasma compartment. Combining Equations 2.23 and 2.24, Equation 2.25 is obtained that expresses the drug's partition coefficient between the tissue and plasma compartment as a function of the steady state volume of distribution.

$$
K=\frac{V_{\mathrm{dss}}-V_{\mathrm{p}}}{V_{\mathrm{t}}}
$$

Equation 2.25 shows that the in vivo steady state volume of distribution is proportional to the drug's partition coefficient between the tissue and the plasma compartment. The schematic illustration of the drug's partitioning in vivo is shown in Figure 2.20. As Figure 2.20 demonstrates, there is another equilibrium process that takes place between the free and bound compartments, which is also important from a pharmaco-dynamic point of view. Based on Brodie's free drug hypothesis [77], the steady state free concentration of the drug molecule in plasma and tissues should be the same when there is no permeability barrier for the drug between compartments and no active transport (efflux or influx) is taking place. The free plasma concentration of a drug can be obtained from the steady state unbound volume of distribution, $V_{\mathrm{du}}$, according to Equation 2.26. 


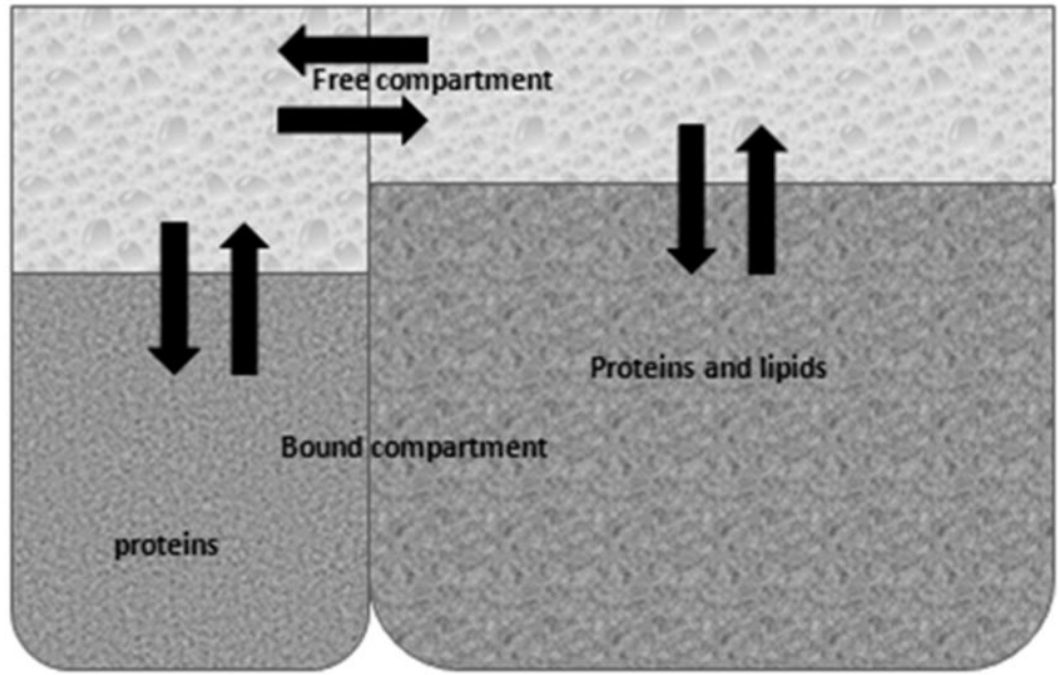

Figure 2.20. The schematic illustration of drug's partitioning in vivo between plasma and tissue compartment as well as the free and bound compartments in plasma and tissues

$$
V_{\mathrm{du}}=\frac{\text { Dose }}{C_{\text {free,plasma }}}
$$

While the average plasma volume and tissue volume can be measured for a living system, it is much more difficult to measure or estimate the volume of the "free" compartment in plasma and tissues. Similar to the expression of $K_{\text {tissue/plasma, we }}$ can have a partition coefficient for the drug between the free and bound

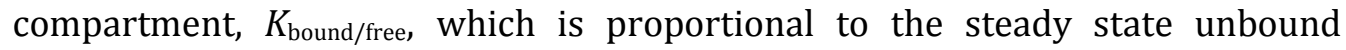
volume of distribution, $V_{\mathrm{du}}$. The in vivo $V_{\mathrm{du}}$ is normally calculated from the $V_{\mathrm{d}}$ and the fraction unbound in plasma, $f_{\mathrm{u}}$, which can be obtained by equilibrium dialysis or ultra-filtration methods [78,79]. Thus, Equation 2.27 shows the expression of $V_{\text {du }}$ from the steady state volume of distribution and the fraction unbound in plasma.

$$
V_{\mathrm{du}}=\frac{V_{\mathrm{dss}}}{f_{\mathrm{u}}}
$$

The plasma compartment contains around 40\% albumin (HSA), 3\% alpha-1-acidglycoprotein (AGP) and $57 \%$ of various immunoglobulins. The tissue compartment contains a large amount of phospholipids besides the various protein components. Thus, the most significant difference between the two compartments is the presence or absence of phospholipids and lipids in general. So, it can be assumed that the major force that drives drug molecules from the plasma compartment to the tissue compartment is their affinity to phospholipids relative to proteins. 


\subsubsection{Modelling steady state volume of distribution}

The hypothesis described above can be investigated using the human clinical steady state volume of distribution and plasma protein binding data [80,81] of known drugs as shown in Table 2.6. Table 2.7 contains measured HSA and IAM binding data [80] using the bio-mimetic HPLC methodology described above. As already published and validated [80], the difference between a compound's affinity to phospholipids and albumin is the major driving force for the drug molecules to partition between the tissue and plasma compartments, therefore showing good correlation to the logarithmic value of the steady state in vivo volume of distribution ( $\left.\log V_{\mathrm{dss}}\right)$ as described by Equation 2.28.

$$
\begin{aligned}
& \log V_{\text {dss }}=0.131 \log K_{(\mathrm{IAM})}-0267 \log K_{(\mathrm{HSA})}-0.305 \\
& (n=70 ; r=0.80 ; s d=0.40)
\end{aligned}
$$

where $n$ is the number of drug molecules listed in Tables 2.6 and 2.7, $r$ is the multiple regression coefficient, and $s d$ is the standard error of the estimate. The $\log K_{\text {(IAM) }}$ and $\log K_{\text {(HSA) }}$ values are obtained as the $e$ power $\log k_{(\mathrm{IAM})}$ and $\log k_{\text {(HSA) }}$ values. Figure 2.21 shows the plot of the $\log V_{\mathrm{dss}}$ values obtained from clinical studies for the 70 marketed drug molecules as a function of the estimated $\log V_{\text {dss }}$ values by Equation 2.28 .

Table 2.6. The human clinical steady state volume of distribution, $V_{\mathrm{dss}}$, plasma protein binding, PPB, and unbound volume of distribution, $V_{\mathrm{du}}$, of a representative set of known drug molecules from the literature $[80,81]$

\begin{tabular}{lcccccc}
\hline DRUG & $\begin{array}{c}\text { Bound in } \\
\text { plasma, }\end{array}$ & $\mathbf{l o g} \frac{\mathbf{P P B}}{\mathbf{1 0 1} \mathbf{P P B}}$ & $\begin{array}{c}\boldsymbol{V}_{\mathbf{d s s}} \\
\mathbf{( L / k g )}\end{array}$ & $\log \boldsymbol{V}_{\mathbf{d s s}}$ & $\begin{array}{c}\boldsymbol{V}_{\mathbf{d u}} \\
\mathbf{( L / k g )}\end{array}$ & $\log \boldsymbol{V}_{\mathbf{d u}}$ \\
\hline Acetaminophen & 1 & -2.00 & 1.0 & -0.02 & 0.97 & -0.01 \\
\hline Acyclovir & 15 & -0.76 & 0.6 & -0.22 & 0.71 & -0.15 \\
\hline Amoxicillin & 18 & -0.66 & 0.2 & -0.67 & 0.26 & -0.58 \\
\hline Ampicillin & 18 & -0.66 & 0.3 & -0.54 & 0.35 & -0.46 \\
\hline Amrinone & 45 & -0.09 & 1.3 & 0.11 & 2.36 & 0.37 \\
\hline Aspirin & 49 & -0.03 & 0.2 & -0.80 & 0.31 & -0.51 \\
\hline Betamethasone & 64 & 0.24 & 1.4 & 0.15 & 3.89 & 0.59 \\
\hline Bromazepam & 60 & 0.17 & 0.9 & -0.04 & 2.28 & 0.36 \\
\hline Bumetanide & 99 & 1.69 & 0.2 & -0.70 & 20.0 & 1.30 \\
\hline Bupivacaine HCl & 95 & 1.20 & 1.0 & 0.02 & 20.0 & 1.32 \\
\hline Ceftazidime & 21 & -0.58 & 0.2 & -0.64 & 0.29 & -0.54 \\
\hline Cephalexin & 14 & -0.79 & 0.2 & -0.59 & 0.30 & -0.52 \\
\hline Chlorpheniramine & 70 & 0.35 & 3.0 & 0.48 & 10.0 & 1.00 \\
\hline Cimetidine & 19 & -0.64 & 1.0 & 0.00 & 1.23 & 0.09 \\
\hline Ciprofloxacin & 40 & -0.18 & 2.2 & 0.34 & 3.67 & 0.56 \\
\hline Clonazepam & 86 & 0.76 & 3.2 & 0.51 & 22.9 & 1.36 \\
\hline Cytarabine & 12 & -0.87 & 2.5 & 0.40 & 2.84 & 0.45 \\
\hline
\end{tabular}




\begin{tabular}{|c|c|c|c|c|c|c|}
\hline DRUG & $\begin{array}{c}\text { Bound in } \\
\text { plasma, \% }\end{array}$ & $\log \frac{P P B}{101-P P B}$ & $\begin{array}{c}V_{\mathrm{dss}} \\
(\mathrm{L} / \mathrm{kg})\end{array}$ & $\log V_{\mathrm{dss}}$ & $\begin{array}{c}V_{\mathrm{du}} \\
(\mathrm{L} / \mathrm{kg})\end{array}$ & $\log V_{d u}$ \\
\hline Diazepam & 99 & 1.69 & 1.1 & 0.04 & 110 & 2.04 \\
\hline Digitoxin & 97 & 1.38 & 0.5 & -0.27 & 18.10 & 1.26 \\
\hline Diltiazem & 78 & 0.53 & 4.5 & 0.65 & 20.45 & 1.31 \\
\hline Diphenhydramine & 63 & 0.22 & 4.5 & 0.65 & 12.16 & 1.09 \\
\hline Doxepin & 83 & 0.66 & 24.0 & 1.38 & 141 & 2.15 \\
\hline Ethosuximide & 1 & -2.00 & 0.7 & -0.15 & 0.72 & -0.14 \\
\hline Felbamate & 23 & -0.53 & 0.8 & -0.12 & 0.97 & -0.01 \\
\hline Finasteride & 90 & 0.91 & 1.1 & 0.04 & 11.0 & 1.04 \\
\hline Flumazenil & 50 & -0.01 & 0.7 & -0.15 & 1.40 & 0.15 \\
\hline Ganciclovir & 1 & -2.00 & 1.1 & 0.04 & 1.11 & 0.05 \\
\hline Gemfibrozil & 97 & 1.38 & 0.1 & -0.85 & 4.67 & 0.67 \\
\hline Glipizide & 98.4 & 1.58 & 0.2 & -0.70 & 12.5 & 1.10 \\
\hline Haloperidol & 92 & 1.01 & 18.0 & 1.26 & 225 & 2.35 \\
\hline Hydrochlorothiazide & 40 & -0.18 & 2.0 & 0.30 & 3.33 & 0.52 \\
\hline Imipramine & 90 & 0.91 & 18 & 1.26 & 180 & 2.26 \\
\hline Indomethacin & 99 & 1.69 & 0.3 & -0.59 & 25.7 & 1.41 \\
\hline Isradipine & 97 & 1.38 & 4.0 & 0.60 & 133 & 2.12 \\
\hline Ketoprofen & 98.7 & 1.63 & 0.1 & -0.96 & 8.46 & 0.93 \\
\hline Lorazepam & 91 & 0.96 & 1.5 & 0.18 & 16.7 & 1.22 \\
\hline Methylprednisolone & 78 & 0.53 & 1.2 & 0.08 & 5.45 & 0.74 \\
\hline Metoclopramide & 40 & -0.18 & 3.0 & 0.48 & 5.00 & 0.70 \\
\hline Metronidazole & 10 & -0.96 & 0.7 & -0.13 & 0.83 & -0.08 \\
\hline Nabumetone & 99 & 1.69 & 0.8 & -0.1 & 79.0 & 1.90 \\
\hline Naproxen & 99.7 & 1.88 & 0.1 & -1.0 & 33.3 & 1.52 \\
\hline Nicardipine & 95 & 1.20 & 1.1 & 0.04 & 2.02 & 1.34 \\
\hline Nifedipine & 96 & 1.28 & 0.8 & -0.10 & 19.6 & 1.29 \\
\hline Nizatidine & 35 & -0.28 & 0.88 & -0.11 & 1.20 & 0.08 \\
\hline Ondansetron & 73 & 0.42 & 1.9 & 0.28 & 7.04 & 0.85 \\
\hline Phenobarbital & 51 & 0.01 & 0.2 & -0.82 & 0.31 & -0.51 \\
\hline Phenytoin & 89 & 0.87 & 0.6 & -0.19 & 5.84 & 0.77 \\
\hline Phenytoin & 89 & 0.87 & 0.6 & -0.19 & 5.82 & 0.76 \\
\hline Piperacillin & 18.5 & -0.65 & 0.18 & -0.74 & 0.22 & -0.66 \\
\hline Piroxicam & 94.5 & 1.16 & 0.1 & -1.00 & 1.82 & 0.26 \\
\hline Prazosin & 95 & 1.20 & 0.5 & -0.3 & 10.0 & 1.00 \\
\hline Prednisolone & 70 & 0.35 & 0.9 & -0.07 & 2.83 & 0.45 \\
\hline Prednisone & 75 & 0.46 & 1.0 & 0.00 & 4.00 & 0.60 \\
\hline Procainamide & 16 & -0.73 & 2.0 & 0.30 & 2.38 & 0.38 \\
\hline Propranolol & 87 & 0.79 & 2.8 & 0.45 & 21.5 & 1.33 \\
\hline Quinidine & 87 & 0.79 & 2.5 & 0.40 & 19.2 & 1.28 \\
\hline
\end{tabular}




\begin{tabular}{lcccccc}
\hline DRUG & $\begin{array}{c}\text { Bound in } \\
\text { plasma, }\end{array}$ & $\mathbf{l o g} \frac{\mathbf{P P B}}{\mathbf{1 0 1 - \mathbf { P B }}}$ & $\begin{array}{c}\boldsymbol{V}_{\mathbf{d s s}} \\
\mathbf{( L / k g )}\end{array}$ & $\log \boldsymbol{V}_{\mathbf{d s s}}$ & $\begin{array}{c}\boldsymbol{V}_{\mathbf{d u}} \\
\mathbf{( L / k g )}\end{array}$ & $\log \boldsymbol{V}_{\mathbf{d u}}$ \\
\hline Quinine & 94 & 1.13 & 1.5 & 0.18 & 25.0 & 1.40 \\
\hline Rifampin & 89 & 0.87 & 1.0 & -0.01 & 8.83 & 0.95 \\
\hline Saquinavir & 98 & 1.51 & 10.0 & 1.00 & 500 & 2.70 \\
\hline Sulfamethoxazole & 62 & 0.2 & 2.0 & 0.3 & 5.30 & 0.72 \\
\hline Terbutaline & 20 & -0.61 & 1.8 & 0.25 & 2.23 & 0.35 \\
\hline Tolbutamide & 96 & 1.28 & 0.1 & -1.00 & 2.50 & 0.04 \\
\hline Trazodone & 93 & 1.07 & 1.0 & 0.00 & 14.3 & 1.15 \\
\hline Trimethoprim & 37 & -0.24 & 1.3 & 0.11 & 2.06 & 0.31 \\
\hline Verapamil & 90 & 0.91 & 4.4 & 0.64 & 44.0 & 1.64 \\
\hline Warfarin & 99 & 1.69 & 0.1 & -0.85 & 14.0 & 1.15 \\
\hline Zidovudine & 2.5 & -1.6 & 1.4 & 0.15 & 1.44 & 0.16 \\
\hline Zolmitriptan & 25 & -0.48 & 7.0 & 0.85 & 9.33 & 0.97 \\
\hline Zolpidem & 92 & 1.01 & 0.7 & -0.17 & 8.00 & 0.93 \\
\hline
\end{tabular}

Another model for the estimation of volume of distribution was published by Lombardo et al. [82]. They applied descriptors of compounds obtained by chromatographic measurements. The model equation contained the Elog $D$ parameter (a chromatographically determined lipophilicity of compounds [28]), a measured or calculated amount of the fraction ionised species at physiological $\mathrm{pH}$ and fraction unbound in plasma obtained by traditional methods. The model provides a good estimate for the in vivo volume of distribution for neutral and basic drugs only; acids were not predicted very well. As it was described earlier, the traditional lipophilicity measures of compounds produce low values for ionised species, regardless of the type of charge, positive or negative. However, the IAM and HSA binding are affected by the type of charge very differently. Positively charged compounds bind much stronger to phospholipids than albumin, thus the volume of distribution of positively charged compounds are large. The negative charge on the molecule has an opposite effect; it reduces the volume of distribution as the compound binds more strongly to albumin and accumulates in the plasma compartment. The introduction of the fraction ionised values of basic compounds in the model accounted for the effect of the positive charge. The effect of the negative charge was not accounted for in the model. Another model was published more recently by Siu et al. [83], who applied the bio-mimetic chromatographic retention using IAM stationary phases. As the IAM retention is very sensitive to the presence of positive charge on the molecule and negatively charged compounds do not bind very strongly to IAM, the model was applicable for basic, neutral and acidic compounds as well. 


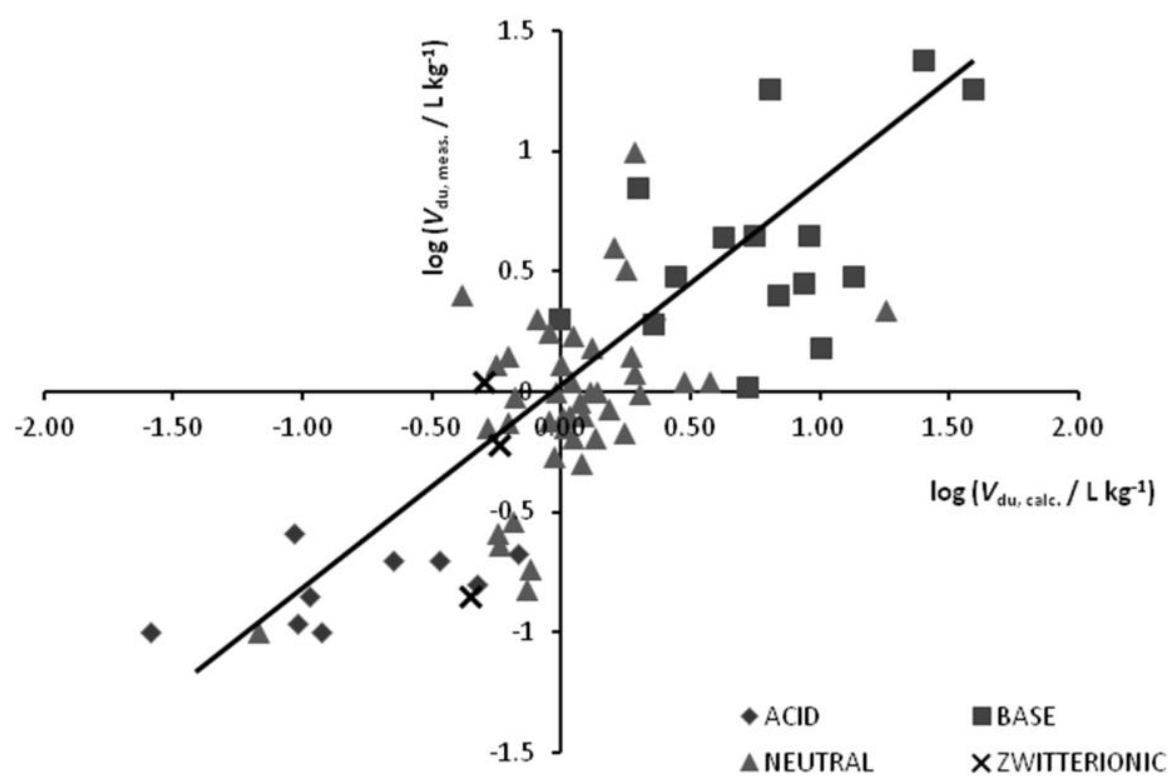

Figure 2.21. The plot of in vivo human clinical steady state volume of distribution and the estimated volume of distribution by Equation 2.28 for 70 known drug molecules

Table 2.7. The measured HPLC based membrane partition (IAM) and human serum albumin (HSA) binding for the known drug molecules [80].

\begin{tabular}{lcccccc}
\hline DRUG & $\log \boldsymbol{k}_{\text {(HSA) }}$ & $\log \boldsymbol{K}_{\text {(HSA) }}$ & HSA, $\%$ & CHIIAM & $\log \boldsymbol{k}_{\text {(IAM) }}$ & $\log \boldsymbol{K}_{\text {(IAM) }}$ \\
\hline Acetaminophen & -0.79 & 0.45 & 14 & 3.97 & 0.6 & 1.83 \\
\hline Acyclovir & -1.25 & 0.29 & 5.38 & -6.98 & 0.1 & 1.1 \\
\hline Amoxicillin & -0.71 & 0.49 & 16.42 & 6.31 & 0.71 & 2.03 \\
\hline Ampicillin & -0.58 & 0.56 & 21.17 & 6.26 & 0.71 & 2.03 \\
\hline Amrinone & 0.28 & 1.32 & 66.14 & 15 & 1.11 & 3.03 \\
\hline Aspirin & -0.23 & 0.79 & 37.34 & -1.73 & 0.34 & 1.41 \\
\hline Betamethasone & 0.09 & 1.09 & 55.58 & 31.73 & 1.88 & 6.55 \\
\hline Bromazepam & 0.32 & 1.38 & 68.46 & 28.45 & 1.73 & 5.63 \\
\hline Bumetanide & 1.15 & 3.15 & 94.3 & 26.08 & 1.62 & 5.05 \\
\hline Bupivacaine HCl & -0.16 & 0.85 & 41.26 & 39.89 & 2.25 & 9.53 \\
\hline Ceftazidime & -1.07 & 0.34 & 7.87 & -5.09 & 0.19 & 1.2 \\
\hline Cephalexin & -0.79 & 0.45 & 14 & -2.27 & 0.32 & 1.37 \\
\hline Chlorpheniramine & 0.18 & 1.2 & 60.95 & 47.19 & 2.59 & 13.34 \\
\hline Cimetidine & -0.58 & 0.56 & 21.17 & 16.59 & 1.18 & 3.26 \\
\hline Ciprofloxacin & -0.09 & 0.91 & 45.02 & 47.82 & 2.62 & 13.73 \\
\hline Clonazepam & 0.51 & 1.66 & 77.11 & 34.77 & 2.02 & 7.54 \\
\hline Cytarabine & -0.38 & 0.69 & 29.8 & -14.86 & -0.26 & 0.77 \\
\hline Diazepam & 1.08 & 2.94 & 93.22 & 37.41 & 2.14 & 8.51 \\
\hline Digitoxin & 0.49 & 1.64 & 76.43 & 27.46 & 1.68 & 5.38 \\
\hline
\end{tabular}




\begin{tabular}{|c|c|c|c|c|c|c|}
\hline DRUG & $\log k_{(\mathrm{HSA})}$ & $\log K_{\text {(HSA) }}$ & HSA, \% & CHIIAM & $\log k_{(\mathrm{IAM})}$ & $\log K_{\text {(IAM) }}$ \\
\hline Diltiazem & 0.14 & 1.15 & 58.46 & 41.63 & 2.33 & 10.33 \\
\hline Diphenhydramine & 0.08 & 1.09 & 55.39 & 44.57 & 2.47 & 11.82 \\
\hline Doxepin & 0.67 & 1.95 & 83.15 & 52.31 & 2.83 & 16.89 \\
\hline Ethosuximide & -0.68 & 0.51 & 17.49 & -5.37 & 0.17 & 1.19 \\
\hline Felbamate & 0.33 & 1.39 & 68.67 & 24.62 & 1.55 & 4.72 \\
\hline Finasteride & 0.45 & 1.57 & 74.73 & 38.86 & 2.21 & 9.09 \\
\hline Flumazenil & -0.58 & 0.56 & 20.86 & 18.37 & 1.26 & 3.54 \\
\hline Ganciclovir & -0.84 & 0.43 & 12.76 & -11.62 & -0.11 & 0.89 \\
\hline Gemfibrozil & 1.29 & 3.64 & 96.08 & 32.89 & 1.93 & 6.91 \\
\hline Glipizide & 1.2 & 3.32 & 95.01 & 21.05 & 1.39 & 4.01 \\
\hline Haloperidol & 0.61 & 1.84 & 81.06 & 45.17 & 2.5 & 12.16 \\
\hline Hydrochlorothiazide & -0.26 & 0.77 & 35.61 & 15.93 & 1.15 & 3.17 \\
\hline Imipramine & 0.67 & 1.95 & 83.15 & 54.14 & 2.91 & 18.37 \\
\hline Indomethacin & 1.82 & 6.17 & 99.5 & 32.46 & 1.91 & 6.77 \\
\hline Isradipine & 1.05 & 2.86 & 92.78 & 40.03 & 2.26 & 9.59 \\
\hline Ketoprofen & 1.57 & 4.8 & 98.35 & 21.94 & 1.43 & 4.17 \\
\hline Lorazepam & 0.96 & 2.62 & 91.1 & 37.29 & 2.14 & 8.46 \\
\hline Methylprednisolone & 0.08 & 1.08 & 54.93 & 32.14 & 1.9 & 6.67 \\
\hline Metoclopramide & 0.02 & 1.02 & 51.4 & 35.35 & 2.05 & 7.74 \\
\hline Metronidazole & -1.25 & 0.29 & 5.38 & -3.32 & 0.27 & 1.31 \\
\hline Nabumetone & 1.15 & 3.16 & 94.32 & 38.42 & 2.19 & 8.91 \\
\hline Naproxen & 1.95 & 7.06 & 99.89 & 22.85 & 1.47 & 4.35 \\
\hline Nicardipine & 1.07 & 2.93 & 93.16 & 45.86 & 2.53 & 12.55 \\
\hline Nifedipine & 0.34 & 1.41 & 69.54 & 29.02 & 1.75 & 5.78 \\
\hline Nizatidine & -0.6 & 0.55 & 20.42 & 17.88 & 1.24 & 3.46 \\
\hline Ondansetron & 0.52 & 1.69 & 77.67 & 37.15 & 2.13 & 8.41 \\
\hline Phenobarbital & -0.27 & 0.76 & 35.22 & 13.58 & 1.04 & 2.84 \\
\hline Phenytoin & 0.47 & 1.6 & 75.47 & 31.55 & 1.87 & 6.5 \\
\hline Phenytoin & 0.61 & 1.84 & 81.04 & 31.09 & 1.85 & 6.36 \\
\hline Piperacillin & -0.74 & 0.48 & 15.44 & 9.43 & 0.85 & 2.35 \\
\hline Piroxicam & 1.47 & 4.36 & 97.71 & 6.75 & 0.73 & 2.08 \\
\hline Prazosin & 0.59 & 1.8 & 80.37 & 31.62 & 1.87 & 6.52 \\
\hline Prednisolone & -0.12 & 0.89 & 43.58 & 27.99 & 1.71 & 5.52 \\
\hline Prednisone & -0.23 & 0.8 & 37.56 & 25.87 & 1.61 & 5 \\
\hline Procainamide & -0.27 & 0.76 & 35.22 & 19.92 & 1.34 & 3.8 \\
\hline Propranolol & 0.29 & 1.33 & 66.51 & 45.12 & 2.5 & 12.13 \\
\hline Quinidine & 0.51 & 1.66 & 76.95 & 44.95 & 2.49 & 12.03 \\
\hline Quinine & 0.5 & 1.65 & 76.65 & 47.11 & 2.59 & 13.29 \\
\hline Rifampin & 0.51 & 1.67 & 77.17 & 35.98 & 2.08 & 7.97 \\
\hline Saquinavir & 1.22 & 3.38 & 95.24 & 43.46 & 2.42 & 11.23 \\
\hline
\end{tabular}




\begin{tabular}{lcccccc}
\hline DRUG & $\log \boldsymbol{k}_{\text {(HSA) }}$ & $\log \boldsymbol{K}_{\text {(HSA) }}$ & HSA, \% & CHIIAM & $\log \boldsymbol{k}_{\text {(IAM) }}$ & $\log \boldsymbol{K}_{\text {(IAM) }}$ \\
\hline Sulfamethoxazole & -0.36 & 0.7 & 30.86 & 31.6 & 1.87 & 6.51 \\
\hline Terbutaline & -0.4 & 0.67 & 28.79 & 16.83 & 1.19 & 3.3 \\
\hline Tolbutamide & 1.28 & 3.59 & 95.95 & 10.24 & 0.89 & 2.44 \\
\hline Trazodone & 0.9 & 2.46 & 89.75 & 36.26 & 2.09 & 8.07 \\
\hline Trimethoprim & -0.19 & 0.82 & 39.49 & 20.77 & 1.38 & 3.96 \\
\hline Verapamil & 0.53 & 1.7 & 77.94 & 41.96 & 2.35 & 10.49 \\
\hline Warfarin & 1.49 & 4.45 & 97.86 & 19.9 & 1.34 & 3.8 \\
\hline Zidovudine & -0.84 & 0.43 & 12.76 & 1.27 & 0.48 & 1.61 \\
\hline Zolmitriptan & -0.36 & 0.7 & 30.61 & 29.71 & 1.79 & 5.97 \\
\hline Zolpidem & 0.43 & 1.54 & 73.58 & 33.91 & 1.98 & 7.24 \\
\hline
\end{tabular}

\subsubsection{Modelling unbound volume of distribution}

The unbound volume of distribution, $V_{\mathrm{du}}$ which is proportional to the drug's distribution between the free and bound compartments, showed good correlation with the sum of the phospholipid and albumin binding as described by Equation 2.29.

$$
\begin{aligned}
& \log V_{\mathrm{du}}=0.24 \log K_{(\mathrm{HSA})}+0.12 \log K_{(\mathrm{IAM})}-0.4 \\
& (n=70 ; r=0.89 ; s d=0.40)
\end{aligned}
$$

The plots of the values found in the literature along with calculated $\log V_{\mathrm{du}}$ values are shown in Figure 2.22. The models described and validated by the data of 130 known drug molecules have been published recently [84].

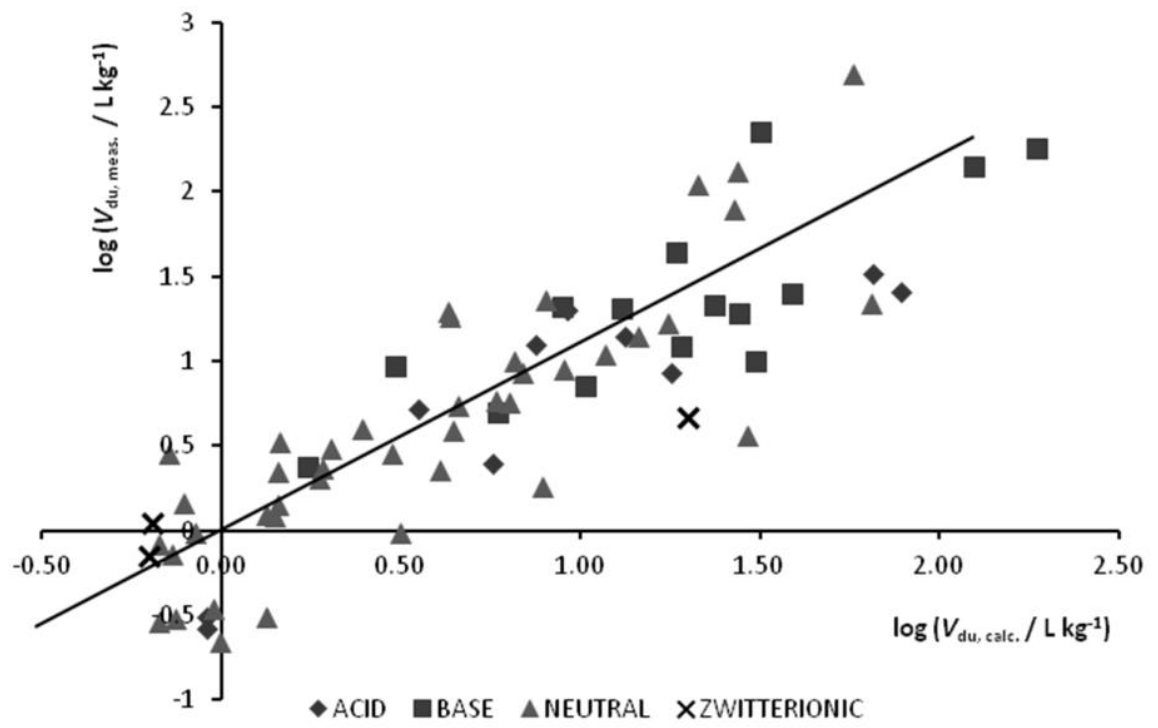

Figure 2.22. The plot of the literature $\log V_{\mathrm{du}}$ and the calculated $\log V_{\mathrm{du}}$ values by Equation 2.29 for 70 known drug molecules 
It is very important to note that the unbound volume of distribution is a good measure of the in vivo efficacious potential of the drug molecule as it is related to the proportion of the free concentration of the drug relative to the administered dose. The unbound volume of distribution is reduced by both albumin and phospholipids binding as expressed by Equation 2.29. The lipophilicity of the compound increases both types of binding. The presence of charge has a significant effect either on the IAM binding (positive charge) or the HSA binding (negative charge). Thus, reducing the octanol/water distribution coefficient $(\log D)$ or reversed phase partition by introducing charge on the molecules will have very little effect on the unbound volume of distribution.

\subsubsection{Dependence of unbound volume of distribution on HSA and IAM binding}

Figures 2.23 and 2.24 show the plots of the logarithmic value of the unbound volume of distribution relative to compounds binding to HSA and IAM, respectively.

Figures 2.23 and 2.24 illustrate that in spite of some compounds having high HSA binding the unbound volume of distribution can still be low (mostly for acidic compounds), while high IAM binding has a great impact on the increase of the unbound volume of distribution.

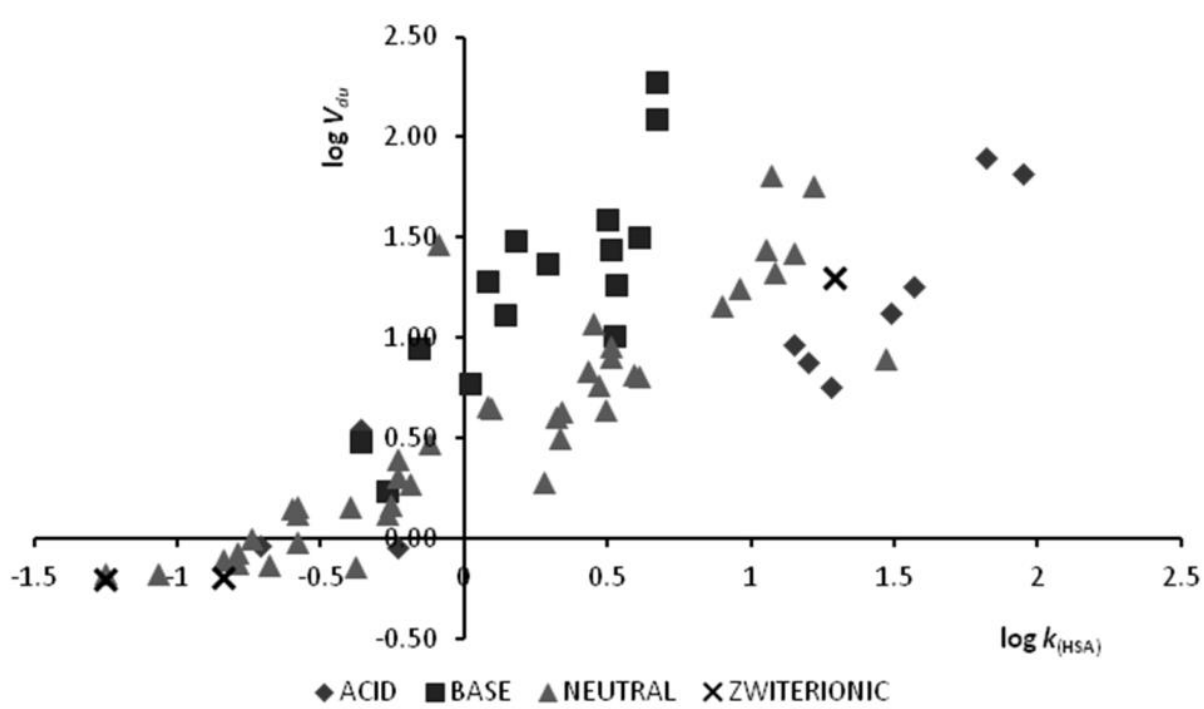

Figure 2.23. The plot of the logarithmic unbound volume of distribution, $\log V_{\mathrm{du}}$ as a function of HSA binding $\left(\log k_{\mathrm{HSA}}\right)$ 


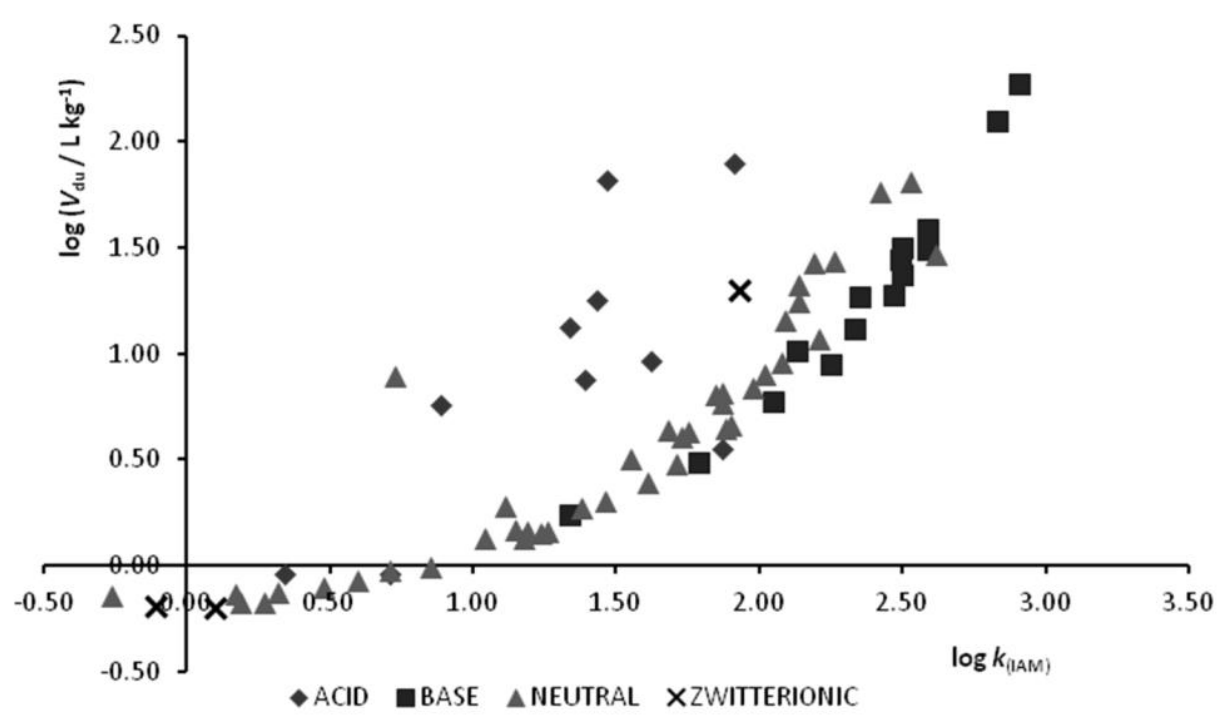

Figure 2.24. The plot of the logarithmic unbound volume of distribution, $\log V_{\mathrm{du}}$ as a function of IAM binding ( $\log k_{\mathrm{IAM}}$ )

These models are the first attempt for estimating parameters such as volume of distribution that can be obtained only from in vivo experiments by using simple in vitro bio-mimetic chromatographic retention time measurements. These models are able to accommodate the charged molecules; moreover they reveal that the presence of positive charge drives drug molecules towards the tissues, as positively charged compounds show extremely strong affinity to phosphatidyl choline relative to the albumin affinity. It is also well known that positively charged drugs usually have a high volume of distribution and strong tissue binding.

Negatively charged compounds bind strongly to albumin and usually have a very low volume of distribution. Obviously, these models are very crude as the in vivo system has been simplified and considered only as two compartments and each compartment is characterised only by one major constituent. The drug distribution in vivo will deviate from the expected distribution if there is a permeability barrier or active transport process is involved that prevents the drug molecule from obtaining thermodynamic equilibrium. Deviation of the drug distribution can be expected if a compound specifically binds to constituents in either compartment which are present in smaller quantities than albumin and phospholipids. The thermodynamic steady state equilibrium of the drug molecule in the in vivo compartments should also be independent from the applied dose, which means that the fraction unbound in plasma, or the volume of distribution is constant even when various amounts of drug is administered. When this is not the case, i.e. non-linear pharmacokinetics are observed, this suggests that the drug binds to specific binding sites that can be saturated by the therapeutically required dose of the drug. This situation can cause significant individual variations in the free plasma concentration in the clinical setting and can cause variable efficacy in different populations of the patients. This phenomenon has been 
observed with drugs that bind specifically and strongly to AGP [85]. As the AGP concentration in the plasma can vary depending on age, gender and disease state [86], the same dose can produce very different free plasma concentrations in different patient populations, thus the efficacious dose has to be monitored for each patient. The measurement of AGP binding is very important in early drug discovery in order to avoid late stage failure due to variable or lack of efficacy in the clinic. The strong specific AGP binding of a compound can be suspected when the AGP binding is much stronger than expected based on the measured lipophilicity.

The physicochemical and bio-mimetic chromatographic partitioning systems are useful for measuring thermodynamic equilibrium or affinity constants of molecules. The volume of distribution even at steady state involves kinetic and rate processes as well. Thus, we have to keep in mind the other form of expressing the volume of distribution which is shown by Equation 2.30.

$$
V_{\mathrm{d}}=t_{1 / 2} \times \text { clearance }
$$

When modelling the steady state volume of distribution we suppose that the plasma concentration is constant over a period of time as the dose is repeated according to the rate of elimination (clearance) and half life $\left(t_{1 / 2}\right)$. The importance of $V_{\mathrm{du}}$ is gaining more and more attention, since the introduction of

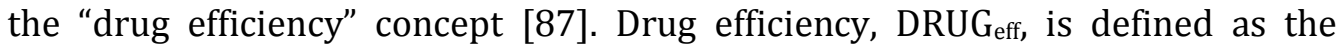
quotient of the free bio-phase concentration and the dose multiplied by 100 . The aim is to achieve the highest free concentration of the drug with a minimum dose. When the free drug hypothesis can be applied, the free plasma concentration is assumed equal to the maximum free bio-phase concentration at the site of action, thus the unbound volume of distribution is in principle the reciprocal value of the drug efficiency. Thus, compounds with the lowest unbound volume of distribution have the highest drug efficiency. It means that modelling the unbound volume of distribution enables us to estimate the drug efficiency early in the drug discovery process, before the selection of a candidate molecule for further development. If the potency of the compound is known and we have an idea as to the number of receptors the drug molecule needs to occupy, then we can estimate the required dose to achieve 50\% receptor/target occupancy in vivo by modelling the unbound volume of distribution.

Besides the drug's distribution between the two major compartments of the body, several attempts have been made to model blood/brain distribution, skin penetration and oral absorption using simple chromatographic measurements of the drug's properties. The IAM retention data of drugs were used to estimate brain to blood partition [88] and compound permeation through the intestinal wall [89]. There are several publications [90-93] about the use of bio-partitioning micellar chromatographic retention to mimic biological in vivo distribution processes. However, when the physico-chemical or bio-mimetic models are applied for the estimation of compounds in vivo behaviour it is very important to keep in mind the limitations of such models. Just because a statistically 
acceptable correlation exists between the measured in vitro and in vivo data does not mean that there will be no outlier compounds from such correlations. We estimate the behaviour of the drug molecules in vivo based on physico-chemical properties ignoring all the possible biological processes, such as active efflux or transport. In spite of the limitation to only thermodynamic processes, these models are still very informative even if they are not predictive for the in vivo situation. They allow us to estimate the compound's behaviour when there is no active transport or efflux processes present. When discrepancies are observed between the estimated and measured in vivo distribution, research can focus on the biological processes that cause such discrepancies.

\subsubsection{General structure-property relationships for lead optimisation}

Another important application of the measured bio-mimetic and physicochemical properties is building structure-property relationships. These relationships enable medicinal chemists to alter the chemical structure and design molecules with desirable in vivo behaviour. These structure-activity studies reveal which part of the molecule interacts with the target receptor or enzyme, and which part can be modified to maintain the potency and improve the in vivo drug distribution. It is also very useful to develop in silico calculations to predict the physico-chemical properties of the molecules before synthesis. Based on a vast amount of accurately measured properties of discovery compounds, a large amount of information is available now for estimating the effect of certain substituents or properties on the compound's in vivo behaviour. The simplest approach is to compare the properties of pairs of molecules which differ only from one substitution. Thus, the effect of the substituents on certain properties can be estimated [94]. The solvation Equation 2.approach also can be used to estimate of effect of H-bond donor and acceptor groups, or the change in polarity on the biological distribution processes (see Table 2.5).

Table 2.8 summarizes the major structure-property relationships observed for the physico-chemical and bio-mimetic properties of drug discovery compounds.

Table 2.8. General structure-property relationships for various physico-chemical and bio-mimetic chromatographic distribution systems

\begin{tabular}{lc}
\hline Distribution system & Structural features \\
\hline Reversed-phase HPLC retention & $\begin{array}{c}\text { In general it is driven by a compound's lipophilicity; H- } \\
\text { bond donor groups reduce partitioning to C-18 much } \\
\text { stronger than the octanol/water lipophilicity; increase of } \\
\text { molecular weight generally increases retention }\end{array}$ \\
\hline Immobilised artificial membrane & It increases with increasing lipophilicity expressed by \\
affinity (IAM retention) & octanol/water partition; positively charged compounds \\
& have strong affinity to IAM and consequently compounds \\
& with high IAM binding are expected to have a large \\
volume of distribution and strong tissue binding
\end{tabular}




\begin{tabular}{lc}
\hline Distribution system & Structural features \\
\hline $\begin{array}{l}\text { Serum albumin binding (HSA } \\
\text { retention) }\end{array}$ & $\begin{array}{c}\text { It increases with increasing lipophilicity expressed by } \\
\text { octanol/water partition; H-bond donor groups do not } \\
\text { reduce binding; negatively charged compounds bind very } \\
\text { strongly; strong binding reduces the volume of } \\
\text { distribution }\end{array}$ \\
\hline $\begin{array}{l}\text { Alpha-1-acid-glycoprotein binding } \\
\text { (AGP retention) }\end{array}$ & $\begin{array}{c}\text { It is increased with increasing lipophilicity; H-bond donor } \\
\text { groups reduce binding; positive charge increases binding }\end{array}$ \\
\hline Blood/brain distribution & $\begin{array}{c}\text { It is increasing with lipophilicity; H-bond donor groups } \\
\text { significantly reduce compounds partitioning into the brain }\end{array}$ \\
\hline Water/skin partition & $\begin{array}{c}\text { It increases with increasing lipophilicity; H-bond donor } \\
\text { groups reduce skin penetration }\end{array}$ \\
\hline Intestinal absorption & $\begin{array}{c}\text { Hydrophilic and large compounds have limited } \\
\text { absorption; optimum lipophilicity is required; both H- } \\
\text { bond donor and acceptor groups reduce absorption }\end{array}$ \\
\hline Volume of distribution & $\begin{array}{c}\text { In general it increases with increasing lipophilicity; } \\
\text { positive charge increases, negative charge decreases } \\
\text { volume of distribution }\end{array}$ \\
\hline Unbound volume of distribution & $\begin{array}{c}\text { It increases with increasing lipophilicity of the uncharged } \\
\text { molecule; H-bond donor groups do not affect the unbound } \\
\text { volume; phospholipid binding has more pronounced effect } \\
\text { on unbound volume of distribution than plasma protein } \\
\text { binding }\end{array}$ \\
\hline
\end{tabular}

\subsection{ALTERNATIVE METHODS}

As discussed above, the HPLC retention times obtained using reversed phase and bio-mimetic stationary phases can be used to measure compound relevant properties for estimating in vivo behaviour. The lipophilicity, protein binding and phospholipid binding affect compound distribution between plasma and tissue compartments and determine the free concentration of the drug at the site of action. There are several other methods that can be used for similar purposes.

\subsubsection{Methods based on the principle of frontal chromatography}

The chromatographic methods described in this chapter are based on the most often used elution mode of chromatography. The frontal mode of chromatography provides another alternative for measuring compound interaction with stationary phases. Frontal chromatography is a mode of chromatography in which the sample is introduced continuously into the column. The sample components migrate through the column at different velocities and eventually break through as a series of fronts. Only the least retained component exits the column in pure form. Thus, they produce continuous detector signals (not peaks as in the elution mode) that form steps as shown in Figure 2.25. The exact shape of a breakthrough curve is mainly determined by the functional form of the underlying equilibrium isotherms of the sample components, but secondary factors such as diffusion and mass-transfer kinetics also have an 
influence. When only one sample component is introduced the molecules occupy the strongest binding sites of the stationary phase first. When all such binding sites are occupied the molecules start adsorbing to the weaker binding sites. The shape of the detector response curve for the breakthrough process can be analysed and the binding strength of the compound to the various binding sites of the stationary phase can be calculated. The application of frontal chromatography for the determination of compound's binding to proteins has been discussed in more detail by Shibukawa et al. [95].

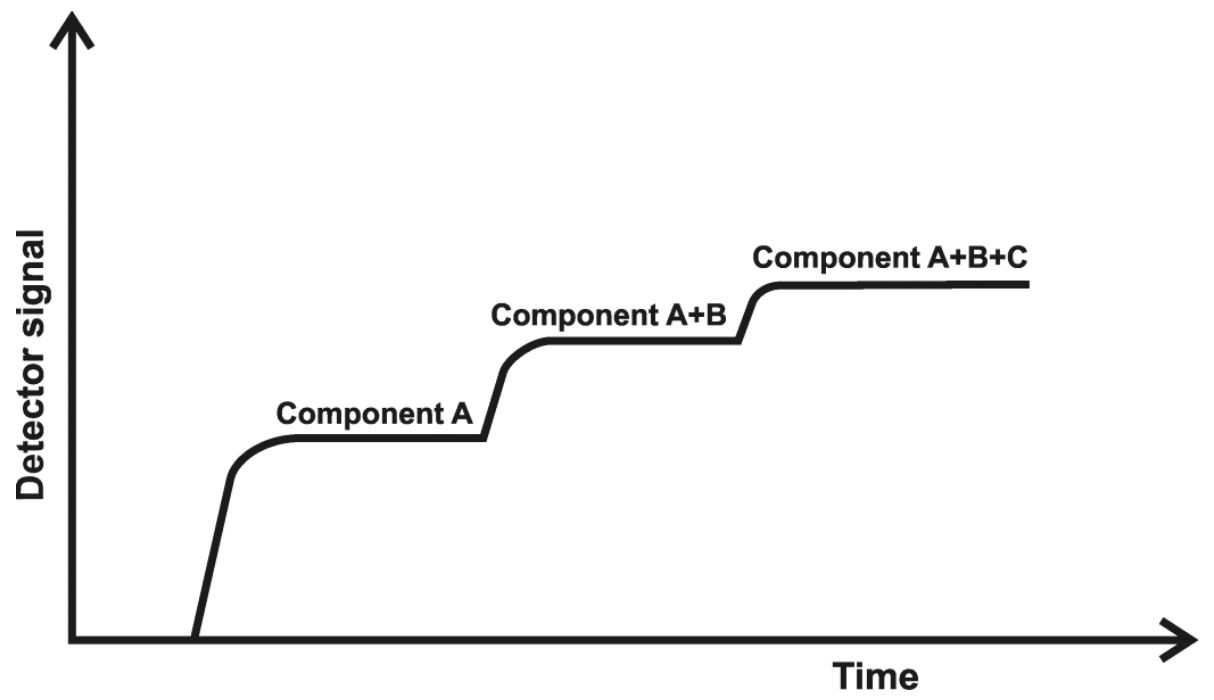

Figure 2.25. Typical chromatogram obtained by frontal chromatography

The method is useful to determine compound binding to various binding sites of the protein. It also provides information about the kinetic aspects of the binding. HPLC instrumentation can be used to carry out the analysis. The calculation of the binding constants requires knowledge of the exact amount of the protein on the stationary phase surface. The column cannot be used immediately for the measurement of another compound, as it needs regeneration to remove the absorbed molecules from the surface. Therefore, the methodology is not applicable at early stages of the drug discovery process when thousands of new compounds have to be characterised in a short period of time. A similar principle is applied to an instrument developed by Biacore ${ }^{\mathrm{TM}}$. The instrument measures the Plasmon resonance on a gold surface where various protein molecules can be immobilised. The sample molecules are introduced on the surface until they fully occupy the binding sites on the immobilised protein. The sample molecules are then slowly removed by washing the surface with buffer (normally at physiological $\mathrm{pH}$ ). Figure 2.26 shows a typical detector response curve. 


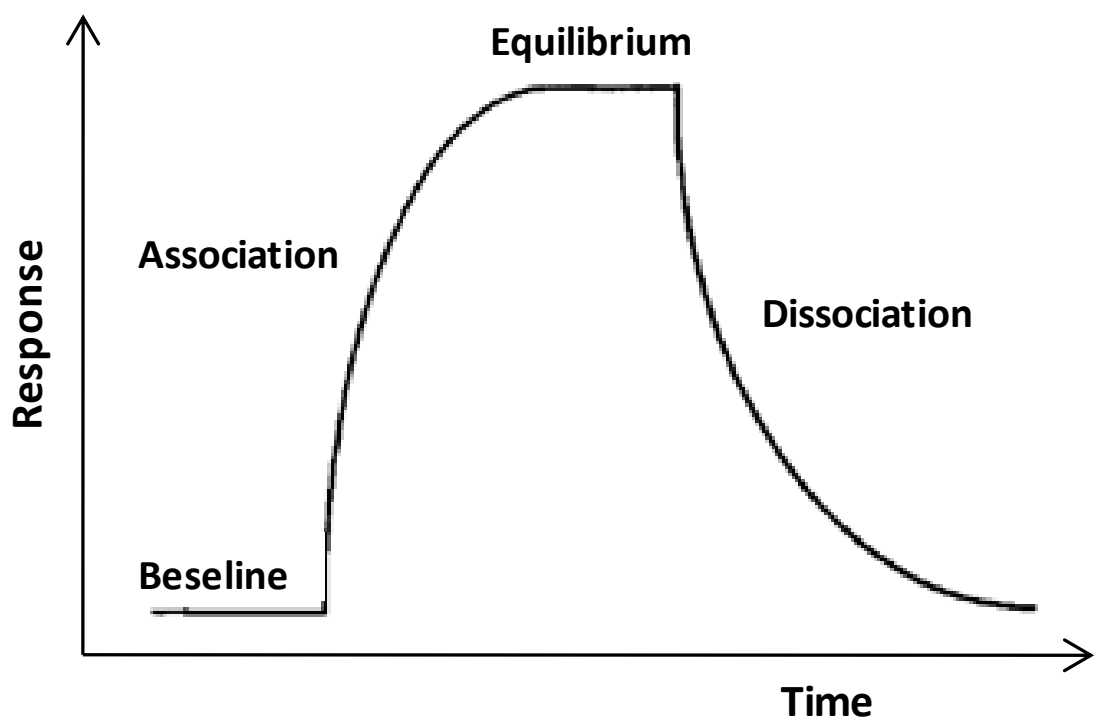

Figure 2.26. Typical Surface Plasmon Resonance (SPR) response obtained by Biacore ${ }^{\mathrm{TM}}$.

From the surface plasmon resonance (SPR) curve shown in Figure 2.26, not only can the binding strength be calculated, but also the onset and offset rates of the binding. Day and Myszka [96] described a procedure in detail for the characterization of compounds binding to the albumin major binding sites. Oravcova et al. [54] have reviewed the application of chromatographic principles in affinity chromatography and size exclusion chromatography. The advantages of these alternative techniques are that they provide more detailed insight into the drug - protein binding identifying the various binding sites on the proteins and the binding kinetics. The understanding of the binding mode and kinetics of a molecule to a protein is very important in the drug design point of view too, as it provides information to medicinal chemists about the structural motives that might be involved in the binding. The Biacore ${ }^{\mathrm{TM}}$ technique is very useful for studying drug - target interactions as very different types of molecules can be immobilised on the gold surface and to be studied by SPR. This technique is very versatile and can be applied at various stages of the drug discovery process. On the other hand, these methods do not provide information about the weaker nonspecific binding sites. Moreover, the possible non-specific binding of the compound to the protein surface actually disturbs these measurements as the one to one association constant cannot be calculated precisely. The individual binding constants are very difficult to use to calculate the free and bound drug concentration in vivo where not only individual binding sites, but a large amount of other proteins are also present. Therefore, these alternative methods provide slightly different types of information and can be applied at a different stage of the drug discovery process. 


\subsubsection{Methods based on solid particles}

Another methodology has been recently introduced using solid supported proteins and lipids (TransilTM technology) in order to improve the throughput of protein and lipid binding measurements [97,98]. Figure 2.27 demonstrates the basic principles of the method.

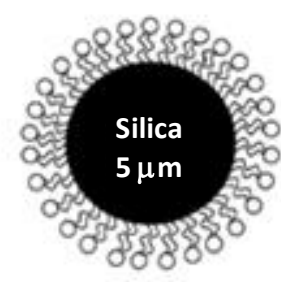

Particle covered with protein or lipid
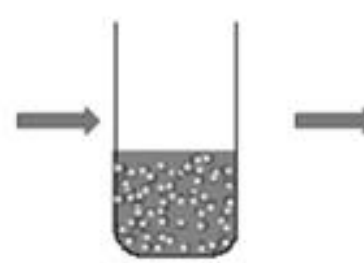

Equilibrate with sample solution

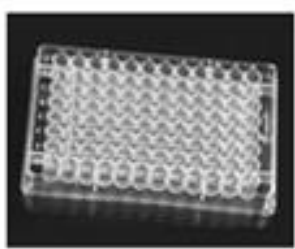

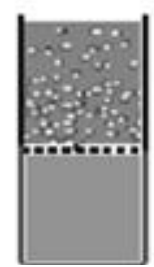

Filter and determine the compound concentration

Figure 2.27. The application of solid supported protein and lipid binding using the Transil ${ }^{\mathrm{TM}}$ technology

The method is applicable at early stages of drug discovery to measure a large number of discovery compounds using robotics and the 96-well plate format. The compound is equilibrated with the protein or lipid immobilised on solid silica particles. After equilibration and filtration, the compound concentration (unbound) can be determined using a generic gradient reversed phase HPLC method. The measurement of the sample solution before equilibration with the solid particles can provide the standard chromatogram. The difference between the peak areas obtained before and after equilibration with the protein and lipid particles indicates the amount of bound compound. The total specific and non-specific binding is obtained by the method; hence, the data can be used directly in models such as volume of distribution. The commercially available PK-MAP software (Bayer AG, Germany) uses the input data obtained by the Transil methodology to estimate a compound's in vivo volume of distribution. Two disadvantages become apparent when comparing this method with the method based on chromatographic retention time. Firstly, one needs to use a chromatographic method twice in order to determine the concentration of the compound before and after the equilibration with the solid particles and calculate the binding constant. Secondly, as the method requires concentration determination, the error increases when the binding of the compound is high, and requires a very sensitive and accurate analytical technique to measure strongly bound compounds. 


\subsubsection{Methods based on equilibrium dialysis and ultra-filtration}

The equilibrium dialysis method for the determination of plasma protein binding and tissue binding can be considered as the traditional gold standard method. The process involves separating the macromolecules, such as plasma proteins and tissue homogenate from low molecular weight compounds in the solution through a semi-permeable membrane as shown in Figure 2.28. The small molecule that is bound to the macromolecule will not be able to cross the membrane. The concentration of the unbound molecules will be the same on both sides of the membrane after equilibrium has been reached. Measuring the concentration of the small molecule in the compartment where no macromolecules are present provides the unbound concentration. The technique is suitable to measure compound binding to a complex biological mixture of proteins and tissue constituents even directly obtained from patients after in vivo administration of the drug molecule. Thus, the result gives a precise measure of the free drug concentration in vivo; this is the biggest advantage of the technique. It can be used in a high throughput manner using a validated rapid equilibrium dialysis device [99]. The device contains the two compartments separated by the membrane in a 96 well plate format.

As the binding measurement is based on the determination of the free concentration, the method gets less accurate at the high binding region. Depending on the accuracy and sensitivity of the concentration determination method (usually HPLC/MS), compounds that are more than $99 \%$ bound to the plasma or tissue cannot be differentiated. The binding equilibrium is distorted due to the dilution process which happens upon the addition of a buffer compartment on the other side of the membrane. The dilution factor has to be included in the calculations.

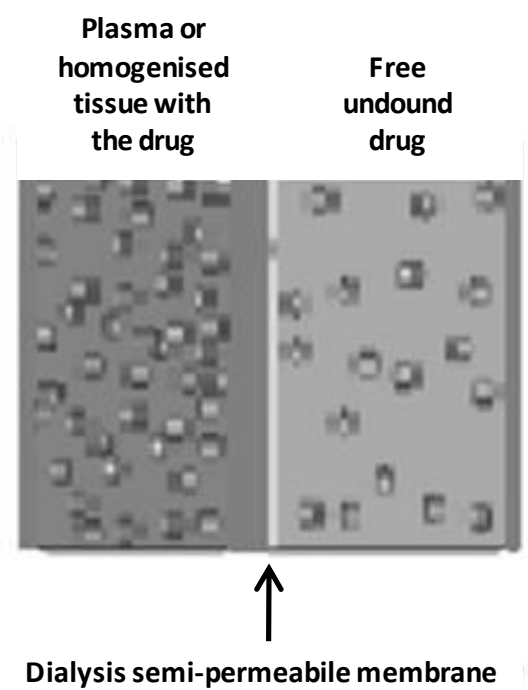

Figure 2.28. The schematic illustration of the equilibrium dialysis measurement. 
The ultra-filtration method is based on a similar principle, but instead of the application of a semi-permeable membrane a filtration procedure is applied for the separation of the unbound compound from the protein bound compound [100]. The concentration of the unbound compound has to be determined in the filtrate. These methodologies can be applied at later stages of the drug discovery process. They provide information on the binding properties of the compounds that is relevant to in vivo circumstances; however it is more difficult to use this information to investigate structure - binding properties as it describes the compound binding to a complex mixture of proteins.

\section{REFERENCES}

1. C.A. Lipinski, F. Lombardo, B.W. Dominy, P.J. Feeny. Experimental and computational approaches to estimate solubility and permeability in drug discovery and development settings. Advanced Drug Delivery Reviews 23 (1997) 325.

2. Avdeef. Absorption and drug development; Solubility, permeability and charge state. Wiley-Interscence, 2003.

3. P.D. Leeson, B. Springthorpe. The influence of drug-like concepts on decisionmaking in medicinal chemistry. Nature Reviews in Drug Discovery 6 (2007) 881890.

4. K. Valko, D.P. Reynolds. High-throughput physicochemical and in vitro ADMET screening; A role in pharmaceutical profiling. American Pharmaceutical review 3 (2005) 83-100.

5. M.P. Gleeson, A. Hersey, D. Montanari, J. Overington. Probing the links between in vitro potency, ADMET and physicochemical parameters. Nature Reviews in Drug Discovery 10 (2011) 197-208.

6. E. Kerns, L. Di. Drug-like properties: concepts, structure design and methods: from ADME to toxicity optimization. Academic Press 2008.

7. H. Van de Waterbeemd, B.C. Jones. Predicting oral absorption and bioavailability. Progress in Medicinal Chemistry. Ed. F.D. King, Oxford, A.W. 41 (2003) 1-59.

8. ACDlogP, Advanced Chemistry Development, www.acdlabs.com.

9. clogP calculator, BioByte, www.biobyte.com.

10. $\log P$ calculator, Chemaxon, www.chemaxon.com.

11. Horvath, W. Melander, I. Molnar. Solvophobic interactions in liquid chromatography with non-polar stationary phases. Journal of Chromatography A 125 (1976) 129-156.

12. L.R. Snyder, J.J. Kirkland, J.W. Dolan. Introduction to modern liquid chromatography. Wiley (2010).

13. V.R. Meyer. Practical high-performance liquid chromatography. Wiley (2010)

14. F. Leroy, B. Presle, F. Verillon, E. Verette. Fast generic-gradient reversed-phase high-performance liquid chromatography using short narrow-bore columns packed with small nonporous silica particles for the analysis of combinatorial libraries. Journal of Chromatographic Sciences 39 (2001) 487-490.

15. Mutton. Fast generic HPLC methods. In Handbook of analytical separations Vol. 1. Separation methods in drug synthesis and purification. Ed. K. Valko, Elsevier (2000) 73-85. 
16. J.M. Call. Liquid-liquid partition coefficients by high-pressure liquid chromatography. Journal of Medicinal Chemistry 18 (1975) 549-552.

17. D.A. Brent, J.J. Sabatka, D.J. Minick, D.J. Henry. A simplified high-pressure liquid chromatography method for determining lipophilicity for structure-activity relationships. Journal of Medicinal Chemistry, 26 (1983) 1014-1020.

18. D.J. Minick, D.A. Brent, J. Frenz. Modeling octanol-water partition coefficients by reversed-phase liquid chromatography. Journal of Chromatography A 461 (1989) 177-191.

19. E. Tomlinson. Chromatographic hydrophobicity parameters in correlation analysis of structure - activity relationships. Journal of Chromatography A 113 (1975) 1-45.

20. T. Braumann, G. Weber, L.H. Grimme. Quantitative structure-activity relationships for herbicides: Reversed-phase liquid chromatographic retention parameter, log $k w$ versus liquid-liquid partition coefficient as model of the hydrophobicity of phenylureas, s-triazines and phenoxycarbonic acid derivatives. Journal of Chromatography 261 (1983) 329-343.

21. R. Kaliszan. Quantitative structure -retention relationships. Analytical Chemistry 64 (1992) 619A-631A.

22. J.G. Dorsey, M.G. Khaledi. Hydrophobicity estimations by reversed-phase liquid chromatography: Implication for biological partition processes. Journal of Chromatography A 656 (1993) 485-499.

23. K. Valko. Measurements of physical properties for drug design in industry. In Handbook of analytical separations Vol. 1. Separation methods in drug synthesis and purification. Ed. K. Valko, Elsevier (2000) 535-583.

24. M.S. Mirrlees, S.J. Moulton, C.T. Murphy, P. Taylor. Direct measurement of octanolwater partition coefficients by high-pressure liquid chromatography. Journal of Medicinal Chemistry 19 (1976) 615-619.

25. S.H. Unger, J.R. Cook, J.S. Hollenberg. Simple procedure for determining octanolaqueous partition, distribution and ionization coefficients by reversed-phase highpressure liquid chromatography. Journal of Pharmaceutical Sciences 67 (1978) 1364-1367.

26. D.J. Minnick, J.H. Frenz, M.A. Patrick, D.A. Brent. A comprehensive method for determining hydrophobicity constants by reversed phase high-performance liquid chromatography. Journal of Medicinal Chemistry 31 (1988) 1923-1933.

27. F. Lombardo, M.Y. Shalaeva, K.A. Tupper, F. Gao, M.H. Abraham. ElogP $\mathrm{P}_{\text {oct: }}$ A tool for lipophilicity determination in drug discovery. Journal of Medicinal chemistry $\mathbf{4 3}$ (2000) 2922-2928.

28. F. Lombardo, M.Y. Shalaeva, K.A. Tupper, F. Gao. Elog $D_{\text {oct: }}$ A tool for lipophilicity determination in drug discovery. 2. Basic and neutral compounds. Journal of Medicinal Chemistry 44 (2001) 2490-2497.

29. M. Harnisch, H.J. Möckel, G. Shulze. Relationship between $\log P_{\text {ow }}$, shake flask values and capacity factors derived from reversed-phase high performance liquid chromatography for n-alkylbenzenes and some oecd reference substances. Journal of Chromatography A 282 (1983) 315-332.

30. K. Valko. General approach for the estimation of octanol/water partition coefficient by reversed-phase high performance liquid chromatography. Journal of Liquid Chromatography 7 (1984) 1405-1424. 
31. K. Valko, P. Slegel. New chromatographic hydrophobicity index $\left(\varphi_{0}\right)$ based on the slope and the intercept of the $\log k^{\prime}$ versus organic phase concentration plot. Journal of Chromatography A 631 (1993) 49-61.

32. K. Valko, C. Bevan, D. Reynolds. Chromatographic hydrophobicity index by fastgradient RP-HPLC: A high throughput alternative to $\log P / \log D$. Analytical Chemistry 69 (1997) 2022-2029.

33. K. Valko. Measurements of lipophilicity and acid/base character using HPLC methods. In "Pharmaceutical profiling in drug discovery for lead selection". Eds. R. Borchardt, E. Kerns, AAPS (2004) Arlington, VA 127-182.

34. E.H. Kerns, L. Di, S. Petusky, T. Kleintop, D. Huryn, O. McConnell, G. Carter. Pharmaceutical profiling method for lipophilicity and integrity using liquid chromatography-mass spectrometry. Journal of Chromatography B 791 (2003) 381-388.

35. S.F. Donovan, M.C. Pescatore. Method for measuring the logarithm of octanol-water partition coefficient by using short octadecyl-poly(vinyl alcohol) high performance liquid chromatography columns. Journal of Chromatography A 952 (2002) 47-61.

36. G. Camurri, A. Zamarella. High-throughput liquid chromatography/mass spectrometry method for the determination of chromatographic hydrophobicity index. Analytical Chemistry 73 (2001) 3716-3722.

37. C.M. Du, K. Valko, C. Bevan, D. Reynolds M.H. Abraham. Rapid gradient RP-HPLC method for lipophilicity determination: A salvation equation based comparison with isocratic methods. Analytical Chemistry 70 (1998) 4228-4234.

38. J.W. Dolan, D.C. Lommen, L.R. Snyder. Drylab ${ }^{\odot}$ computer simulation for highperformance liquid chromatographic method development II. Gradient elution. Journal of Chromatography A 485 (1998) 91-112.

39. Canals, K. Valko, E. Bosch, A.P. Hill, M. Roses. Retention of ionisable compounds on HPLC. 8. Influence of mobile-phase $\mathrm{pH}$ change on the chromatographic retention of acids and bases during gradient elution. Analytical Chemistry 73 (2001) 4937-4945.

40. R. Kaliszan, P. Haber, T. Baczek, D. Siluk, K. Valko. Lipophilicity and $\mathrm{p} K_{\mathrm{a}}$ estimates from gradient high-performance liquid chromatograhy. Journal of Chromatography A 965 (2002) 117-127.

41. P. Wiczling, P. Kawczak, A. Nasal, R. Kaliszan. Simultaneous determination of $\mathrm{p} K_{\mathrm{a}}$ and lipophilicity by gradient RP-HPLC. Analytical Chemistry 78 (2006) 239-249.

42. R. Kaliszan, T.A.G. Noctor, I.W. Wainer. Quantitative structure - enantioselective retention relationships for the chromatography of 1,4-benzodiazepines on a human serum albumin based HPLC chiral stationary phase: An approach to the computational prediction of retention and enantioselectivity. Chromatographia 33 (1992) 546-550.

43. T.A.G. Noctor, M.J. Diaz-Perez, I.W. Wainer. Use of human serum albumin-based stationary phase for high performance liquid chromatography as a tool for rapid determination of drug plasma protein binding. Journal of Pharmaceutical Sciences 82 (1993) 675-676.

44. P.R. Tiller, I.M. Mutton, S.J. Lane, C.D. Bevan. Immobilized human serum albumin: Liquid chromatography/mass spectrometry as a method of determining drugprotein binding. Rapid Communications in Mass Spectrometry 9 (1995) 261-263.

45. F. Beaudry, M. Coutu, N.K. Brown. Determination of drug-plasma protein binding using human serum albumin chromatography column and multiple linear regression model. Biomedical Chromatography 13 (1999) 401-406. 
46. K. Valko, S.B. Nunhuck, C.D. Bevan, M.H. Abraham, D.P. Reynolds. Fast gradient HPLC method to determine compounds binding to human serum albumin. Relationships with octanol/water and immobilized artificial membrane lipophilicity. Journal of Pharmaceutical Sciences 92 (2003) 2236-2248.

47. C. Bertucci, M. Bartolini, R. Gotti, V. Andrisano. Drug affinity to immobilized target bio-polymers by high-performance liquid chromatography and capillary electrophoresis. Journal of Chromatography B 797 (2003) 111-129.

48. L. Buchholz, C.H. Cai, A. Cleton, J. Brodfuehrer, L. Cohen. Evaluation of the human serum albumin column as discovery screening tool for plasma protein binding. European Journal of Pharmaceutical Sciences 15 (2002) 209-215.

49. N. Kratochwil, W. Huber, F. Muller, M. Kansy, P.R. Gerber. Predicting plasma protein binding of drugs - revisited. Current Opinion in Drug Discovery \& Development 7 (2004) 507-512.

50. G. Colmenarejo, A. Alvarez-Pedraglio, J-L. Lavandera. Cheminformatic models to predict binding affinities to human serum albumin. Journal of Medicinal Chemistry 44 (2001) 4370-4378.

51. R.C. Jewell, K.L.R. Brouwer, P.J.C. McNamara. $\alpha 1$-acid glycoprotein highperformance liquid chromatography column (Enantiopac) as a screening tool for protein binding. Journal of Chromatography 487 (1989) 257-264.

52. Rapti. Evaluation of $\alpha_{1}$-acid glycoprotein(AGP) binding measurements by high performance liquid chromatography \& ultrafiltration Methods, Project Report, Drug Discovery MSci, School of Pharmacy, University of London, 2006.

53. R. Kaliszan, A. Nasal, M. Turowski. Binding site for basic drugs on alpha-1-acid glycoprotein as revealed by chemometric analysis of biochromatographic data. Biomedical Chromatography 9 (1995) 211-215.

54. J. Oravcova, B. Bohs, W. Lindner. Drug-protein studies trends in analytical and experimental methodology. Journal of Chromatography B 677 (1996) 1-28.

55. Pidgeon, U.V. Venkataram. Immobilized artificial membrane chromatography: Supports composed of membrane lipids. Analytical Biochemistry 176 (1989) 36-47.

56. Taillardat-Bertschinger, C. Marca Martinet, P.-A. Carrupt, M. Reist, G. Caron, R. Fruttero, B. Testa. Molecular factors influencing retention on immobilized artificial membranes (IAM) compared to partitioning in liposomes and n-octanol. Pharmaceutical Research 19 (2002) 729-737.

57. Taillardat-Bertschinger, P. A. Carrupt, F. Barbato, B. Testa. Immobilised Artificial membrane HPLC in Drug Research. Journal of Medicinal Chemistry 46 (2003) 655665.

58. Giaginis, A. Tsantili-Kakoulidou. Alternative Measures of lipophilicity: From octanol/water partitioning to IAM retention. Journal of Pharmaceutical Sciences 97 (2008) 2984-3004.

59. B.H. Stewart, F.Y. Chung, B. Tait, C. John, O.H. Chan. Hydrophobicity of HIV protease inhibitors by immobilized artificial membrane chromatography: application and significance to drug transport. Pharmaceutical Research 15 (1998) 1401-1406.

60. K. Valko, C.M. Du, C. Bevan, D. Reynolds, M.H. Abraham. Rapid-gradient HPLC method for measuring drug interactions with immobilized artificial membrane: Comparison with other lipophilicity measures. Journal of Pharmaceutical Sciences 89 (2000) 1085-1096.

61. K. Valko, C.M. Du, C. Bevan, D.P. Reynolds, M.H. Abraham. Rapid method for the estimation of octanol/water partition coefficient (log $P_{\text {oct }}$ ) from gradient RP-HPLC 
retention and a hydrogen bond acidity term $\left(\Sigma \alpha_{2}{ }^{\mathrm{H}}\right)$. Current Medicinal Chemistry 8 (2001) 1137-1146.

62. Hansch, T. Fujita. $\rho-\sigma-\pi$ analysis. A method for the correlation of biological activity and chemical structure. Journal of American Chemical Society 86 (1964) 16161626.

63. M.H. Abraham. Scales of solute hydrogen bonding: Their construction and application to physicochemical and biochemical processes. Chemical Society Reviews 22 (1993) 73-83.

64. M.H. Abraham, H.S. Chadha, G.S. Whitin, R.C. Mitchell. Hydrogen bonding. 32. An analysis of water-octanol and water-alkane partitioning and the $\Delta \log \mathrm{p}$ parameter of seiler. Journal of Pharmaceutical Science 83 (1994) 1085-1100.

65. A.Nasal, M. Sznitowska, A. Bucinski, R. Kaliszan. Hydrophobicity parameter from high-performance liquid chromatography on an immobilized artificial membrane column and its relationship to bioactivity. Journal of Chromatography A, 692 (1995) 83-89.

66. M.H. Abraham, H.S. Cadha, R.A.E. Leiato, R.C. Mitchell, W.J. Lambert,R. Kaliszan, A. Nasal, P. Haber. Determination of solute lipophilicity, as $\log \mathrm{P}$ (octanol) and $\log \mathrm{P}($ alkane) using poly(styrene-divinylbenzene) and immobilized artificial membrane stationary phases in reversed-phase high-performance liquid chromatography. Journal of Chromatography A 766 (1997) 35-47.

67. K. Valko, M. Plass, C. Bevan, D. Reynolds, M.H. Abraham. Relationship between the chromatographic hydrophobicity indices and solute descriptors obtained by using several reversed-phase, diol, nitrile, cyclodextrin and immobilised artificial membrane bonded high-performance liquid chromatography columns. Journal of Chromatography A 797 (1998) 41-55.

68. C.M. Du, K. Valko, C. Bevan, D. Reynolds, M.H. Abraham. Characterizing the selectivity of stationary phases and organic modifiers in reversed-phase high performance liquid chromatographic systems by a general solvation equation using gradient elution. Journal of Chromatographic Science 38 (2000) 503-511.

69. C.M. Du, K. Valko, C. Bevan, D. Reynolds, M.H. Abraham. Rapid method for estimating octanol-water partition coefficient (log $P_{\text {oct }}$ ) from isocratic RP-HPLC and a hydrogen bond acidity term (A). Journal of Liquid Chromatography \& Related Technologies 24 (2001) 635-649.

70. M.H. Abraham, J.A. Platts in "The Blood/Brain Barrier and Drug Delivery to CNS". Editors D. J. Begley, M. W. Bradbury, J. Kreuter. Marcel Dekker, New York. NY 2000, 9-33.

71. Y.H. Zhao, J. Le, M.H. Abraham, A. Hersey, P.J. Eddershaw, C.N. Luscombe, D. Butina, G. Beck, B. Sherborne, I. Cooper, J.A. Platts. Evaluation of human intestinal absorption data and subsequent derivation of a quantitative structure - activity relationship (QSAR) with the Abraham descriptors. Journal of Pharmaceutical Sciences 90 (2001) 749-784.

72. M.H. Abraham, H.S. Chadha, R.C. Mitchell. The factors that influence skin penetration of solutes. Journal of Pharmacy and Pharmacology 47 (1995) 8-16.

73. M.H. Abraham, M. Roses. Hydrogen bonding. 38. Effect of solute structure and mobile phase composition on reversed-phase high-performance liquid chromatographic capacity factors Journal of Physical Organic Chemistry 7 (1994) 672-684. 
74. D.M. Cimpean, C.F. Poole. Systematic search for surrogate chromatographic models of biopartitioning processes. Analyst 127 (2002) 724-729.

75. M. Hidalgo-Rodriguez, E. Fuguet, C. Rafols, M. Roses. Estimation of biological properties by means of chromatographic systems: evaluation of the factors that contribute to the variance of biological - chromatographic correlations. Analytical Chemistry 82 (2010) 10236-10245.

76. M. Roses, D. Bolliet, C.F. Poole. Comparison of solute descriptors for predicting retention of ionic compounds (phenols) in reversed-phase liquid chromatography using the solvation parameter model. Journal of Chromatography A 829 (1998) 2940.

77. B.B. Brodie, H. Kurtz, L.J. Schanker. The importance of dissociation constants in lipid solubility on influencing the passage of drugs into the cerebrospinal fluid. Journal of Pharmacology and Experimental Therapeutics 130 (1960)20-25.

78. M.J. Banker, T.H. Clark, J.A. Williams. Development and validation of a 96-well equilibrium dialysis apparatus for measuring plasma protein binding. Journal of Pharmaceutical Sciences 92 (2003) 967-974.

79. S.S. Singh, J. Mehta. Measurement of drug-protein binding by immobilized human serum albumin-HPLC and comparison with ultrafiltration. Journal of Chromatography B 834 (2006) 108-116.

80. F. Hollosy, K. Valko, A. Hersey, S. Nunhuck, Gy. Keri, C. Bevan. Estimation of volume of distribution in humans from high throughput HPLC-based measurements of human serum albumin and immobilized artificial membrane partitioning. Journal of Medicinal Chemistry 49 (2006) 6958-6971.

81. F. Lombardo, R.S. Obach, F.M. DiCapua, G.A. Bakken, J. Lu, D.M. Potter, F. Gao, M.D. Miller, Y. Zhang. A hybrid mixture discriminant analysis- random forest computational model for the prediction of volume of distribution of drugs in human. Journal of Medicinal Chemistry 49 (2006) 2262-2267.

82. F. Lombardo, R.S. Obach, M.Y. Shalaeva, F. Gao. Prediction of Volume of distribution values in humans for neutral and basic drugs using physicochemical measurements and plasma protein binding data. Journal of Medicinal Chemistry 45 (2002) 2867-2876.

83. X. Sui, J. Sun, H. Li, Y. Wang, J. Liu, X. Liu, W. Zhang, L. Chen, Z. He. Prediction of volume of distribution values in human using immobilized artificial membrane partitioning coefficients, the fraction of compounds ionized and plasma protein binding data. European Journal of Medicinal Chemistry 44 (2009) 4455-4460.

84. K. Valko, S.B. Nunhuck, A.P. Hill. Estimating unbound volume of distribution and tissue binding by in vitro HPLC-based human serum albumin and immobilised artificial membrane - binding measurements. Journal of Pharmaceutical Sciences 100 (2011) 849-862.

85. T. Fournier, N.-N. Medjoubi, D. Porquet. Alpha-1-acid glycoprotein. Review. Biochimica et Biophysica Acta - Protein Structure and Molecular Enzymology 1482 (2000) 157-171.

86. M.K. Grandison, F.D. Boudinot. Age-related changes in protein binding of drugs. Implications for therapy. Clinical Pharmacokinetics 38 (2000) 271-290.

87. S. Braggio, D. Montanari, T. Rossi, E. Ratti. Drug efficiency: a new concept to guide lead optimization programs towards the selection of better clinical candidates. Expert Opinion on Drug Discovery 5 (2010) 609-618. 
88. T. Salminen, A. Pulli, J. Taskinen. Relationship between immobilized artificial membrane chromatographic retention and the brain penetration of structurally diverse drugs. Journal of Pharmaceutical and Biomedical Analysis 15 (1997) 469477.

89. J. Kotecha, S. Shah, I. Rathod, G. Subbaiah. Relationship between immobilized artificial membrane chromatographic retention and human oral absorption of structurally diverse drugs. International Journal of Pharmaceutics 333 (2007) 127135.

90. L. Escuder-Gilabert, J.J. Martinez-Pla, S. Sagrado, R.M. Villanueva-Camanas, M.J. Medina-Hernandez. Bio-partitioning micellar separation methods: modelling drug absorption. Journal of Chromatography B 797 (2003) 21-35.

91. Y. Martin-Biosca, S. Torres-Cartas, R.M. Villanueva-Camanas, S. Sagrado, M.J. Medina-Hernandez. Biopartitioning micellar chromatography to predict blood to lung, blood to liver, blood to fat and blood to skin partition coefficients of drugs. Analytica Chimica Acta 632 (2009) 296-303.

92. L. Escuder-Gilabert, M. Molero-Monfort, R.M. Villanueva-Camanas, S. Sagrado, M.J. Medina-Hernandez. Potential of biopartitioning micellar chromatography as an in vitro technique for predicting drug penetration across the blood-brain barrier. Journal of Chromatography B 807 (2004) 193-201.

93. S. Torres-Cartas, Y. Martin-Biosca, R.M. Villanueva-Camanas, S. Sagrado, M.J. Medina-Hernandez. Biopartitioning micellar chromatography to predict mutagenicity of aromatic amines. European Journal of Medicinal Chemistry 42 (2007) 1396-1402.

94. A.G. Leach, H.D. Jones, D.A. Cosgrove, P.W. Kenny, L. Ruston, P. MacFaul, J.M. Wood, N. Colclough, B. Law. Matched molecular pairs as a guide in the optimization of pharmaceutical properties; a study of aqueous solubility, plasma protein binding and oral exposure. Journal of Medicinal Chemistry 49 (2006) 6672-6682.

95. Shibukawa, Y. Kuroda, T. Nakagawa. High performance frontal analysis for drugprotein binding study. Journal of Pharmaceutical and Biomedical analysis 18 (1999) 1047-1055.

96. Y.S.N. Day, D.G. Myszka. Characterizing a drug's primary binding site on albumin. Journal of Pharmaceutical Sciences 92 (2003) 333-343.

97. Loidl-Stahlhofen, T. Hartmann, M. Schottner, C. Rohring, H. Brodowsky, J. Schmitt, J. Keldenich. Multilamellar liposomoes and solid-supported lipid membranes (TRANSIL): Screening of lipid-water partitioning toward a high-throughput scale. Pharmaceutical Research 18 (2001) 1782-1788.

98. J. Schuhmacher, C. Kohlsdorfer, K. Buchner, T. Brandenburger, R. Kruk. Highthroughput determination of the free fraction of drugs strongly bound to plasma proteins. Journal of Pharmaceutical Sciences 93 (2004) 816-830.

99. N.J. Waters, R. Jones, G. Williams, B. Sohal. Validation of a rapid dialysis approach for the measurements of plasma protein binding. Journal of Pharmaceutical Sciences 97 (2008) 4586-4595.

100. J.B. Whitlam, K.F. Brown. Ultrafiltration in serum protein binding determinations. Journal of Pharmaceutical Sciences 70 (1981) 146-150. 